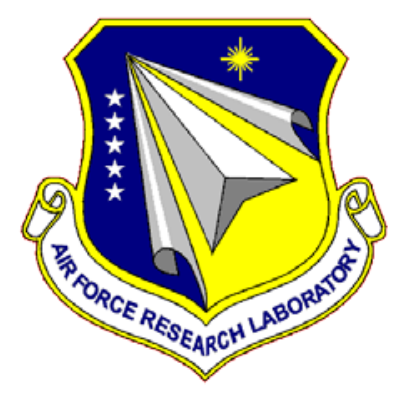

\title{
AFRL-RY-WP-TR-2015-0066
}

\section{TUNABLE HIGH BRIGHTNESS SEMICONDUCTOR SOURCES}

Robert Bedford, Saima Husaini, Charles Reyner, and Tuoc Dang

Optoelectronics Technology Branch

Aerospace Components \& Subsystems Division

MAY 2015

Final Report

Approved for public release; distribution unlimited.

See additional restrictions described on inside pages

\section{STINFO COPY}

\author{
AIR FORCE RESEARCH LABORATORY \\ SENSORS DIRECTORATE \\ WRIGHT-PATTERSON AIR FORCE BASE, OH 45433-7320 \\ AIR FORCE MATERIEL COMMAND \\ UNITED STATES AIR FORCE
}




\section{NOTICE AND SIGNATURE PAGE}

Using Government drawings, specifications, or other data included in this document for any purpose other than Government procurement does not in any way obligate the U.S. Government. The fact that the Government formulated or supplied the drawings, specifications, or other data does not license the holder or any other person or corporation; or convey any rights or permission to manufacture, use, or sell any patented invention that may relate to them.

This report was cleared for public release by the USAF 88th Air Base Wing (88 ABW) Public Affairs Office (PAO) and is available to the general public, including foreign nationals. Copies may be obtained from the Defense Technical Information Center (DTIC) (http://www.dtic.mil).

AFRL-RY-WP-TR-2015-0066 HAS BEEN REVIEWED AND IS APPROVED FOR PUBLICATION IN ACCORDANCE WITH ASSIGNED DISTRIBUTION STATEMENT.

//Signature//

ROBERT G. BEDFORD

Program Manager

Optoelectronic Components Branch

Aerospace Components \& Subsystems Division

\author{
//Signature// \\ JONAHIRA R. ARNOLD, Chief \\ Optoelectronic Components Branch \\ Aerospace Components \& Subsystems Division
}

//Signature//

JASON V. PAUL, Maj. USAF

Deputy Division Chief

Aerospace Components \& Subsystems Division

Sensors Directorate

This report is published in the interest of scientific and technical information exchange, and its publication does not constitute the Government's approval or disapproval of its ideas or findings.

*Disseminated copies will show “//Signature//” stamped or typed above the signature blocks. 


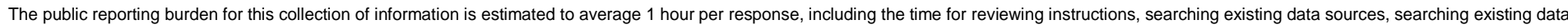

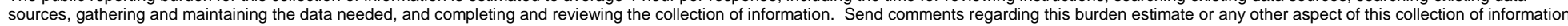

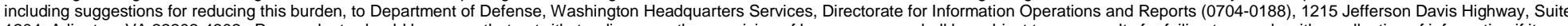

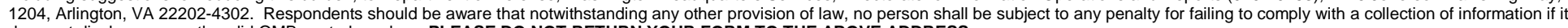
does not display a currently valid OMB control number. PLEASE DO NOT RETURN YOUR FORM TO THE ABOVE ADDRESS
1. REPORT DATE (DD-MM-YY)
2. REPORT TYPE
May 2015
Final
3. DATES COVERED (From - To)
5 November 2010 - 1 February 2015

4. TITLE AND SUBTITLE

TUNABLE HIGH BRIGHTNESS SEMICONDUCTOR SOURCES

5a. CONTRACT NUMBER

In-house

5b. GRANT NUMBER

5c. PROGRAM ELEMENT NUMBER 62204F

6. AUTHOR(S)

Robert Bedford, Saima Husaini, Charles Reyner, and Tuoc Dang (AFRL/RYDH)

5d. PROJECT NUMBER

202D

5e. TASK NUMBER

11

5f. WORK UNIT NUMBER

Y00R

7. PERFORMING ORGANIZATION NAME(S) AND ADDRESS(ES)

Optoelectronics Technology Branch (AFRL/RYDH)

Aerospace Components \& Subsystems Division

Air Force Research Laboratory, Sensors Directorate

PERFORMING ORGANIZATION REPORT NUMBER

AFRL-RY-WP-TR-2015-0066

Wright-Patterson Air Force Base, OH 45433-7320

Air Force Materiel Command, United States Air Force

9. SPONSORING/MONITORING AGENCY NAME(S) AND ADDRESS(ES)

Air Force Research Laboratory

10. SPONSORING/MONITORING AGENCY ACRONYM(S)

Sensors Directorate

AFRL/RYDH

Wright-Patterson Air Force Base, OH 45433-7320

Air Force Materiel Command

1. SPONSORING/MONITORING AGENCY REPORT NUMBER(S)

United States Air Force

AFRL-RY-WP-TR-2015-0066

12. DISTRIBUTIONIAVAILABILITY STATEMENT

Approved for public release; distribution unlimited.

13. SUPPLEMENTARY NOTES

The U.S. Government is joint author of the work and has the right to use, modify, reproduce, release, perform, display or disclose the work. PAO case number 88ABW-2015-2055, Clearance Date 22 April 2015. Report contains color.

14. ABSTRACT

This final report documents research included within the Tunable High Brightness Semiconductor Sources work unit includes several technology advancements. First, theoretical advances in mid-IR type-I quantum well laser efficiency improvement are presented utilizing deep understanding of Auger recombination effects and how to change them through proper laser design. Experimental results are presented to confirm this effect at a wavelength of approximately 2 microns. Future directions are presented, including metamorphic buffer layer grown material, interfacial misfit layers, etc. 'Second, a surface-emitting distributed feedback type-II quantum well laser is introduced and experimental advances are presented. Thirdly, progress in graphene-based saturable absorbers and reverse saturable absorbers are presented. Finally, advances in solder technology for optoelectronics (including, but not limited to mid-IR lasers) is presented with novel high surface quality indium, and intracavity difference frequency generation.

15. SUBJECT TERMS

quantum well laser, infrared laser, optoelectronics

16. SECURITY CLASSIFICATION OF:

\begin{tabular}{|l|l|l|}
\hline a. REPORT & b. ABSTRACT & c. THIS PAGE \\
\hline
\end{tabular}

Unclassified Unclassified Unclassified
17. LIMITATION OF ABSTRACT: SAR
18. NUMBER OF PAGES 92 19a. NAME OF RESPONSIBLE PERSON (Monitor)

Robert Bedford

19b. TELEPHONE NUMBER (Include Area Code) (937) XXX-XXXX 


\section{Table of Contents}

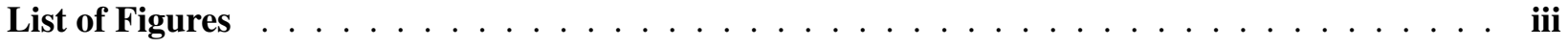

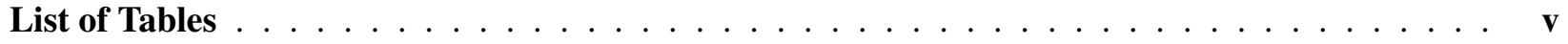

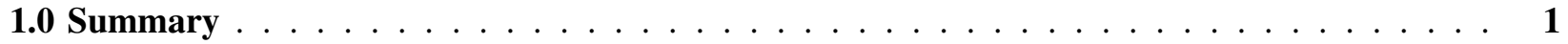

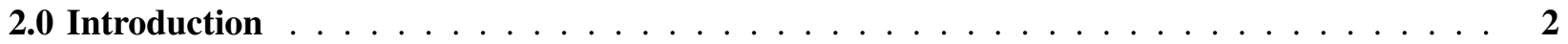

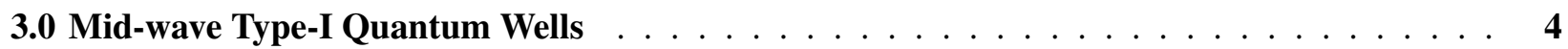

3.1 Type-I Method Auger Engineering Principals . . . . . . . . . . . . . . . . . . . 4

3.2 Engineering Approaches to Auger Processes . . . . . . . . . . . . . . . 6

3.3 Growth and Fabrication $\ldots \ldots \ldots \ldots \ldots \ldots$

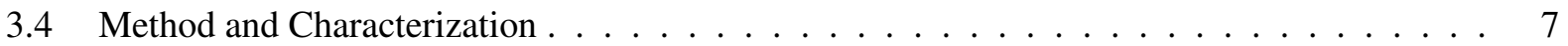

3.4 .1 Test Structures . . . . . . . . . . . . . . . . . . . . . . . . . 11

3.5 Type-I Results and Discussion . . . . . . . . . . . . . . . . . . . . . . 12

3.6 IMF-grown Materials . . . . . . . . . . . . . . . . . . . . . . . . 15

3.6.1 Limitations of the IMF for SWIR active regions . . . . . . . . . . . . . . . 15

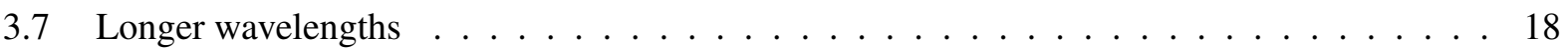

3.7.1 Type-I Method: Strain Engineering . . . . . . . . . . . . . . . . . 20

$3.7 .2 \quad$ Practical Implications . . . . . . . . . . . . . . . . . . . . . . 24

4.0 Type-II Quantum Well Surface-emitting Distributed Feedback Laser . . . . . . . . . . . . 25

4.1 Type-II Method: Active Region Modeling . . . . . . . . . . . . . . . . . . . . 25

4.1 .1 Double-Confined Structure . . . . . . . . . . . . . . . . . . . 26

$4.1 .2 \quad$ Single-Confined Structure . . . . . . . . . . . . . . . . . . . 27

4.2 Device Design . . . . . . . . . . . . . . . . . . . . . . 27

4.3 Type-II Device Modeling Results and Discussion . . . . . . . . . . . . . . . . . . . . . . 29

4.3 .1 Introduction . . . . . . . . . . . . . . . . . . . . . 29

4.3.2 Coupled Differential Equations . . . . . . . . . . . . . . . . . . . . . 29

4.3.3 Approximate Estimates of Grating Parameters . . . . . . . . . . . . . . . . . . 30

4.3.4 General Computation Method . . . . . . . . . . . . . . . . 31

4.3.5 Shooting-Method Numerical Technique . . . . . . . . . . . . . . . 32

4.3 .6 Optical Gain Parameters . . . . . . . . . . . . . . . . . . 33

4.3 .7 Grating Parameters . . . . . . . . . . . . . . . . . . 34

4.3 .8 Device Designs . . . . . . . . . . . . . . . . . . . . . 34

4.3.9 Modeling Results and Discussion . . . . . . . . . . . . . . . . . 35

4.4 Fabrication and Packaging . . . . . . . . . . . . . . . . . . 37

$4.4 .1 \quad$ Fabrication Steps $\ldots \ldots \ldots \ldots \ldots \ldots$

4.4 .2 Grating Patterning . . . . . . . . . . . . . . . . . . 41

4.4 .3 Dry Etching GaSb Materials . . . . . . . . . . . . . . . . . . . 44

5.0 Broadband Nonlinear Components $\ldots \ldots \ldots \ldots \ldots$

5.1 Graphene Polymer Composites . . . . . . . . . . . . . . . . 46

5.1 .1 Preparation Techniques . . . . . . . . . . . . . . . . 46

5.1.2 Graphene Optical Characterization Methods . . . . . . . . . . . . . . . . . 47

5.1 .3 Demonstration of Composite Structures . . . . . . . . . . . . . . . . . . 54

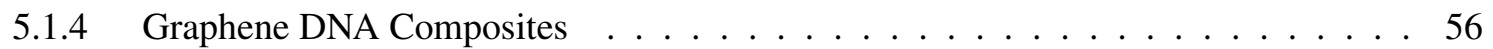


5.2 Graphene Saturable Absorber Mirrors . . . . . . . . . . . . . . . . . . . 58

5.2 .1 Design of Saturable Absorber Mirrors . . . . . . . . . . . . . . . . 58

5.2.2 Characterization of Materials and Saturable Absorber Mirrors . . . . . . . . . . . 59

5.2.3 Growth and Fabrication . . . . . . . . . . . . . . . . . . . . . . 62

5.2.4 Graphene Mode-locked Laser Characterization . . . . . . . . . . . . . . . 63

6.0 Associated Research . . . . . . . . . . . . . . . . . . . . . . . . . . . 67

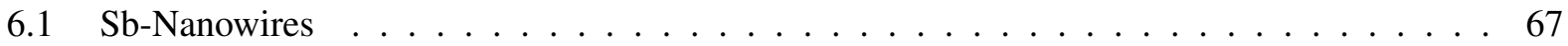

6.2 Laser Packaging . . . . . . . . . . . . . . . . . . . . . . . . . . . 69

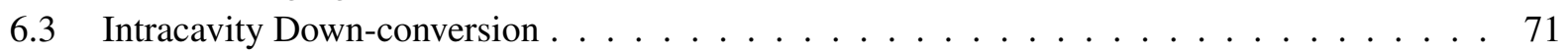

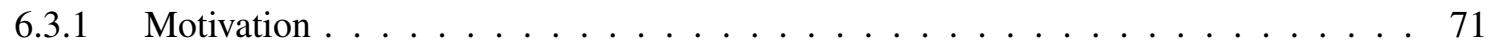

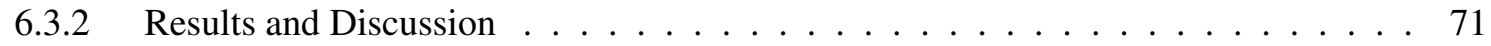

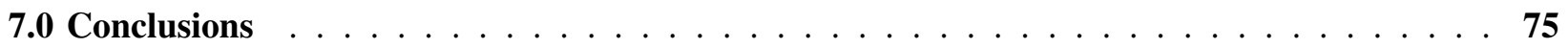

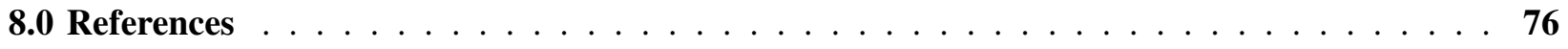

List of Symbols, Abbreviations, and Acronyms $\ldots \ldots \ldots \ldots$ 


\section{List of Figures}

Figure

Page

1 Carrier lifetime dependence on conduction band offset. . . . . . . . . . . . 6

2 Auger lifetime and coefficients calculated at room temperature. . . . . . . . . 8

3 Energy diagrams of the three structures $\mathrm{HB}, \mathrm{MB}$, and LB structures. . . . . . . . . . . 8

$4 \quad$ RHEED schematic . . . . . . . . . . . . . . . . . . . . . . 9

5 Planview AFM of InGaAsSb surface with varying concentrations $\ldots \ldots \ldots \ldots$

6 Reflectivity and photoluminescence of several samples . . . . . . . . . . . . . 11

7 Example of high-resolution X-ray diffraction (HR-XRD) $\Omega-2 \Theta$ rocking curves. . . . . . . . 12

8 Laser power characteristics comparing devices with various barrier heights. . . . . . . . 13

9 Measured and calculated threshold for various temperatures. . . . . . . . . . . . . 14

10 Layer structure and image of IMF structure . . . . . . . . . . . . . . . . . . . 16

11 Time-resolved photoluminescence and STEM images of structure . . . . . . . . . . . 17

12 Simulated Auger and spontaneous lifetimes for $\sim 3.5 \mu$ m designed quantum wells. . . . . . 19

13 Equivalent thresholds and simulated Auger lifetimes for $\lambda \sim 2.9 \mu$ m devices . . . . . . . . . 21

14 Measured PL from $77 \mathrm{~K}$ to $300 \mathrm{~K}$ for devices operating at approximately $3 \mu \mathrm{m}$. . . . . . . 22

$15 \mathrm{XRD}$ for metamorphic buffer layer grown material designed to operate at approximately $3 \mu \mathrm{m} .22$

16 Metamorphic Buffer Layer grown material designed at approximately $3 \mu \mathrm{m}$. . . . . . . 23

17 Improvements to wall-plug efficiency. . . . . . . . . . . . . . . 25

18 Standard type-II quantum well laser band schematic . . . . . . . . . . . . . . . . 26

19 Conceptual approach to the double-confined structure . . . . . . . . . . . . . . 27

20 Absorption and wavefunction calculations for type-II quantum well active region . . . . . 28

21 Influence of hole-well thickness . . . . . . . . . . . . . . . . . . . . . 29

22 Grating and field images from the design with $150 \times 4000 \mu \mathrm{m}^{2}$ stripe size and 7-quantum

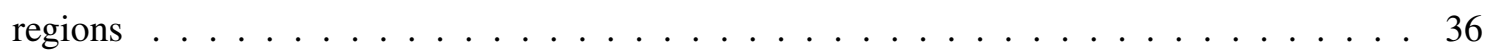

Simulated slit scans of the far field, near field and virtual waist . . . . . . . . . . . . 38

24 Proposed type-II SE-DFB processing steps. . . . . . . . . . . . . . . . . . . 39

25 Rayleigh-Sommerfeld diffraction intensity patterns for coherent proximity lithography . . . 42

26 Microscope image of best exposure result of e-beam lithography . . . . . . . . . . . . . . 43

27 Etch-rates and etch roughnesses of GaSb etched using ICP . . . . . . . . . . . . . . . 44

28 Schematic showing preparation of the graphene-poly vinyl alcohol (PVA) composites. . . . 46

29 Optical transmission of graphene-based solutions . . . . . . . . . . . . . . . . . . . . . . . . . .

30 Raman spectra of graphene composite films . . . . . . . . . . . . . . . . . . . . . . . . . . . . .

31 I-scan measurement technique schematic . . . . . . . . . . . . . . . . . . 48

32 Plots showing optical limiting in the dispersions developed using $1.7 \mathrm{mg} / \mathrm{ml}$ graphene-sodium dodecyl sulfate $($ SDS) solution. . . . . . . . . . . . . . . . . . . . . . . . 49

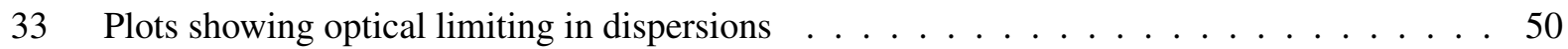

34 Evidence of variable optical limiting behavior based on path length . . . . . . . . . . . 51

35 The energy transmission and fluence out is plotted versus input fluence . . . . . . . . . 52

36 The energy transmission for various pulse-widths and wavelengths . . . . . . . . . . 52

37 Concentration dependance of linear and nonlinear material transmittance in films . . . . . 53 
38 Path length dependance of linear and nonlinear material transmittance (comparing film to

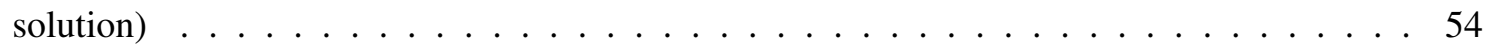

39 Demonstration of tunable nonlinear components with concentration . . . . . . . . . 55

40 Steps for graphene - DNA (G-DNA) composite processing . . . . . . . . . . . 56

$41 z$-scan characterization of G-DNA films of various concentrations $\ldots \ldots \ldots$. . . . . . . 57

42 Calculated field-confinement varying spacer layer . . . . . . . . . . . . . . . . 59

43 Calculated nonlinear reflectance for various GSAM designs . . . . . . . . . . . . 60

44 Linear and Raman graphene measurements from the visible through the near-infrared region 60

45 Nonlinear reflection measurement system . . . . . . . . . . . . . . . . . . 62 62

$46 \quad$ V-cavity schematic and power characteristics . . . . . . . . . . . . 63

47 Optical spectrum, autocorrelation, and RF spectrum f . . . . . . . . . . . 65

48 Narrowed RF spectrum indicating stable mode-locking . . . . . . . . . . . . . . . 66

49 Analysis of $\mathrm{Sb}$-nanowires on $\mathrm{GaSb} \ldots \ldots \ldots$. . . . . . . . . . . . . . . 68

50 AFM and Crossectional SEM of indium deposited above and below homologous temperature 70

51 T-VECSEL schematic, experiment image, and theory . . . . . . . . . . . 72

52 Theoretical/experimental DFG idler frequencies demonstrating tunability from 5.3 and $5.5 \mu \mathrm{m} .73$ 


\section{List of Tables}

Table

Page

1 IMF PL Decay Characterization . . . . . . . . . . . . . . . . 17

2 Wall plug efficiency characterization . . . . . . . . . . . . . 24

3 Calculated and assumed waveguide parameters for two structures sent to be grown. . . . . 34

$4 \quad$ Numerical results of laser modeling . . . . . . . . . . . . . . . . . . . 35 


\subsection{SUMMARY}

This final report on research included within the Tunable High Brightness Semiconductor Sources work unit [202D1100] includes several technology advancements. First, theoretical advances in mid-wave infrared (MWIR) type-I quantum well laser efficiency improvement are presented utilizing deep understanding of Auger recombination effects and how to change them through proper laser design. Experimental results are presented to confirm this effect at a wavelength of approximately $2 \mu \mathrm{m}$. Future directions are presented, including metamorphic buffer layer grown material. Second, a surface-emitting distributed feedback type-II quantum well laser is introduced and experimental advances are presented. Graphene-based nonlinear optical materials are introduced for their application to broad-band laser technologies, as well as associated functions such as sensor protection. Advances in solder technology for optoelectronics (including, but not limited to mid-IR lasers) is presented with some novel high density indium with extraordinary surface and crystal features. Finally, a unique intracavtiy difference-frequency generation (DFG) semiconductor laser is introduced and demonstrated. 


\subsection{INTRODUCTION}

There are few light-weight, efficient, optical sources available in the $\sim 2-4 \mu \mathrm{m}$ band. Presently, the two biggest competitors in this arena are Cr-doped solid-state lasers, and non-linear frequency converted sources (both of which are being pursued within Sensors Directorate (AFRL/RY)). The former, while reasonably efficient ( $\sim 15 \%$ wall plug efficiency, not including cooling), is bulky, and is most effective in the 2.3-3 $\mu \mathrm{m}$ band. The latter necessitates the complexity of a multi-element system, though does allow broad spectral tuning utilizing a single source. There are other solid-state approaches that may be suitable for direct longer wavelength generation, they are complicated by cryogenic cooling which adds to the package complexity and reduces overall efficiency. While semiconductor lasers have addressed some of this spectral region, with a few notable exceptions, they require cryogenic cooling, affecting the necessary power budget for the component.

There are important infrared countermeasures (IRCM)+ bands I, II and IV within this region that are presently addressed only through nonlinear frequency conversion. Sources operating in the MWIR region also are important for laser radar (LADAR) applications because the wavelength range between 3.5 and $3.9 \mu \mathrm{m}$ is where diffraction, scattering, and divergence are optimally balanced. For similar reasons, this same band would be critical to ground-based secure free-space communication systems; these applications remain critical to attain Department of Defense (DoD) needs. If these applications are enabled with compact, efficient, in-band mid-IR sources, it is possible to proliferate sensor protection (layered sensing: assured global access), search and target identification (layered sensing: engagement quality information), as well as the jamming (layered sensing: spectrum dominance and control) in these same bands, not only to the highest value assets within the theater of operations, but to lower value assets as well, assuring complete sensor coverage of the battlespace. Furthermore, with such sensor abundance, sensor networking and multi-modal sensing become an added benefit. This may be embodied by bi-static in-band illumination/sensing, chemical sensing, etc.

short-wave infrared (SWIR) and MWIR lasers are proving to have many applications in the sensing community, including remote chemical sensing of numerous organic compounds such as methane[1], an ideal spectral regime for low-altitude laser radar[2] and, if sufficiently bright, infrared countermeasures. Although there are several potentially successful approaches to developing semiconductor sources in the short-wave and mid-wave infrared, as one approaches the 2-4 $\mu \mathrm{m}$ band, challenges exist that must be overcome for successful adoption as a product. Type-I quantum well lasers are the mainstay of the semiconductor laser industry, having dominated early on and have been used to achieve a broad wavelength regime. However, performance of type-I quantum wells suffer at longer wavelengths, due primarily to an increased Auger recombination rate. We chose to approach the problem by addressing the Auger recombination mechanisms directly and demonstrate this capability in the region of $\lambda=2 \mu \mathrm{m}$.

In Section 5.0, we introduce graphene as a nonlinear optical element. We present the exploration of nonlinear behavior of graphene composites in Section 5.1, which has applications to sensor protection. For additional laser functionality, the "open cavity" nature of the vertical external cavity surface emitting laser (VECSEL) provides an extremely attractive platform as tunable, high power laser sources with nearly diffraction-limited beams. [3, 4] The VECSEL is considered to be a hybrid between diode pumped solid state laser and a semiconductor laser. Essentially it is comprised of a vertical cavity surface emitting laser (VCSEL) with the top distributed Bragg reflector (DBR) removed and replaced by an external curved mirror. The subsequent insertion of an appropriate saturable absorber with fast response time leads to the production of ultrafast, 
high power pulses. Section Section 5.2 presents design and experimental demonstration of graphene-based absorbers to mode-lock VECSELs. Graphene saturable absorbers have previously been used to mode-lock both solid state and fiber lasers. However, unlike a VECSEL, these are able to withstand reasonable absorption without any detrimental effects. VECSELs have been more challenging to integrate with graphene due to their high sensitivity to losses. In an attempt to solve this issue, we report the combine the saturable absorption demonstrated and studied by layer(s) of graphene with the antiresonant saturable absorber. [5, 6] This results in a low loss cavity with a high saturation fluences as well as ultrashort pulses.

Such a laser system is therefore quite attractive for several Air Force related applications such as LADAR, IRCM, and chemical sensing and identification. Air Force applications necessitate compact and efficient sources that address multiple functions since air platforms limit the accommodation of many individual components for each mission. Thus, VECSELs with tunable pulse widths, wavelengths, and repetition rates provide the necessary functionality to carry out several missions in one compact device.

Because VECSELs can access a wide range of wavelengths, their use in LADAR systems for acquiring, tracking, and classifying various targets is possible. By using pulsed transmission, which may help minimize thermal and environmental propagation effects, lasers operating in the ultraviolet (UV) to infrared can detect any scattered radiation and process them in a laser radar receiver to remotely determine target characteristics. Further, proactive IRCM using LADAR systems can allow for protection from weapon tracking systems. Various chemical and biological agents possess characteristic resonances which span over a wide wavelength range. Using a mode-locked laser in a cavity ring down spectroscopy, where the center wavelength may be tuned to the particular chemical of interest, can be of great advantage to rapid chemical analysis systems. This would allow one to probe necessary vibrational modes of the individual constituents of the molecular species in question. VECSELs designed for the mid-to-far infrared regime are especially attractive since many of the chemical and biological absorption characteristics exist in that region.

Additionally in Section 6.0, we report related work that, while may not have been associated with the physical principals proposed to develop tunable high-brightness semiconductor sources, do affect the general performance of lasers, or can be used in concert with semiconductor sources for the purposes of enhancing desired effects for specific applications. First, in Section 6.1 we present a unique method to produce metallic nano-particles that on a semiconductor surface that has unique polarization characteristics. We then introduce related work we've pursued in order to obtain very low-void, high quality solder layers in Section 6.2. In Section 6.3 we present the work of an AFRL/RY funded entrepreneurial research project on intracavity nonlinear down-conversion in which we demonstrated tunable MWIR lasers with a cavity-enhanced nonlinear conversion efficiency. Finally, we make some concluding remarks in Section 7.0. 


\subsection{MID-WAVE TYPE-I QUANTUM WELLS}

Short-wave and mid-wave infrared lasers are proving to have many applications in the sensing community, including remote chemical sensing of numerous organic compounds such as methane[1], an ideal source for low-altitude laser radar[2] and, if sufficiently bright, infrared countermeasures. Although there are several potentially successful approaches to developing semiconductor sources in the short-wave and mid-wave infrared, as one approaches the 2-4 $\mu \mathrm{m}$ band, challenges exist that must be overcome for successful adoption as a product.

Within the last 15 years, The quantum cascade laser (QCL) has proven to be a very capable semiconductor laser platform, owing to the ability to rely on an engineered approach to intersubband transitions, making them very attractive for lower energy lasers. However, these lasers are limited by band offset in the conduction band $[7,8]$, and the desire for higher energy sources is progressing toward more exotic material system $[9,10]$. Equally as promising are type-II quantum well lasers which rely on electrons and holes being confined in adjacent layers[11]. When these layers are sufficiently thin, their electronic wavefunctions have significant overlap, and are well suited for wavelengths above $\sim 2.8 \mu \mathrm{m}$, although somewhat restricted at the longer wavelengths, compared to QCLs due to band filling[12]. In the short-wave and mid-wave infrared wavelengths, their low $T_{0}$ necessitates low temperatures to achieve appreciable powers, even with 10 or more quantum wells[11].

Type-I quantum well lasers can address the 2-4 $\mu \mathrm{m}$ wavelength band with the gallium antimonide (GaSb) material system. Although immature relative to III-P and III-As systems, GaSb provides a reasonably flexible platform for the (AlGaIn)(AsSb) material system, and allows for various bandgaps, refractive index-, and strain-profiles within the active region. Ternary InGaAs quantum wells[13] are suitable for $\lambda \lesssim 2.1 \mu \mathrm{m}$, but are generally limited to low compositions of indium and thin layers to avoid excessive strain. At longer wavelengths, it becomes necessary to utilize the InGaAsSb quaternary quantum well to control strain and achieve higher indium concentration[14]. Utilizing a "quinternary" barrier material of InAlGaAsSb, room temperature $\mathrm{cw}$ operation well over $3 \mu \mathrm{m}$ has been demonstrated[15, 16]. Performance of this material system suffers at longer wavelengths, due primarily to increased Auger recombination.

We chose to approach the problem by addressing the Auger recombination mechanisms directly and demonstrate this capability in the region of $\lambda=2 \mu \mathrm{m}$. In Section 3.1, we address the principles of Auger recombination mitigation, and outline our approach. In Section 3.3, we describe the growth and fabrication of broad area lasers used to measure the Auger carrier losses, followed by our results and discussion in Section 3.5.

\subsection{Type-I Method Auger Engineering Principals}

Type-I quantum well lasers are attractive at wavelengths below about $2 \mu \mathrm{m}$, but at longer wavelengths, they tend to suffer from increased Auger losses, as the photon energies become resonant with higher subbands. This effect is nominally proportional to $N_{t h}^{3}$, where $N_{t h}$ is the threshold carrier density. A reduction in $N_{t h}$ has been the most common approach to achieving efficient lasers. Even then, it has been shown that the proportionality constant (know as the "Auger coefficient", and typically denoted as $C$ ) dramatically increases above $2 \mu \mathrm{m}[17]$.

Unfortunately, an increased differential gain (thus practically reducing $N_{t h}$ ) is often coupled with increased 
Auger coefficient, diminishing the returns of the lower operating carrier density. The carrier density dependence of the Auger coefficient is not commonly considered, which results in incomplete, and sometimes incorrectly designed structures. In our calculations, we avoid this empirical relation, and rather calculate the Auger processes with Simulase ${ }^{\circledR}$, a product of Nonlinear Control Strategies, Inc. (NLCSTR), which utilizes many-body theory, determining a carrier-dependent lifetime $(\tau)$ which we use to evaluate quantum well design. The typical approximations used for Auger scattering equations which utilize simplified Coulomb coupling matrix elements typically predict values for Auger recombination which deviate from actual values by an order of magnitude.

For the purposes here, we use semiconductor Bloch equations, quantum Boltzmann scattering to determine dephasing of $P$, the macroscopic optical polarization, calculated from the microscopic polarizability, as well as the lineshape of absorption/gain[18]. Spontaneous emission is determined with semiconductor

luminescence equations with higher order Coulomb correlation source terms. These rigors avoid issues with lineshape errors in $N$ and $T$ as well as spectral positions.

Critical for narrow bandgap structures, Auger recombination calculations utilize scattering equations in second Born-Markov approximation with fully resolved $k$-, spin-, and subband-summations as well as z-Integrals. This technique is more thoroughly discussed in Reference 19. This gain model requires only input defining structural layout (layer widths, compositions), and basic bulk bandstructure parameters (e.g. Luttinger parameters, band gaps, and band offset[20]). When comparing to experiments, the only free parameters are therefore inhomogeneous broadening, Shockley-Read-Hall (SRH) recombination, internal efficiency (wrapped into pump absorption) and internal modal optical loss. These "free parameters" may be measured independently, and will vary depending on e.g. interface quality and compositional control.

We then apply these to the steady-state values of a simple set of rate equations. Above threshold, these are approximately:

$$
\begin{aligned}
\frac{d N}{d t} & =\eta_{i} \frac{J}{M}-\left(\frac{1}{\tau_{a}}+\frac{1}{\tau_{b}}+\frac{1}{\tau_{c}}\right) N-v_{g} g N_{p}, \\
\frac{d N_{p}}{d t} & =-\frac{N_{p}}{\tau_{p}}+v_{g}(\Gamma g-\alpha) N_{p} .
\end{aligned}
$$

In these equations, $N$ is the 2-D carrier density, $\eta_{i}$ is dominated by the optical absorption, and is analogous to internal quantum efficiency. The carrier excitation by the pump is denoted as $J\left(J \equiv \Phi / \hbar \omega_{p}\right)$, and $M$ is the number of quantum wells in the structure, assuming generated carriers are distributed evenly throughout the wells. The SRH, spontaneous, and Auger lifetimes are given by $\tau_{a, b, c}$, respectively. The quantum well gain is $g, N_{p}$ is the 2-D photon density, $\tau_{p}$ is the geometric photon lifetime, $\Gamma$ is the 3-D mode overlap with all of the quantum wells, and $\alpha$ is the waveguide loss (composed of everything except the active region absorption). In arriving at Equations 1 and 2, we ignore carrier exchange between the barriers and wells due to the low barrier carrier density, accounting for these carriers as if they were generated directly in the well. We also ignore the contribution of spontaneous emission to photon density. Our detection scheme did not have sufficient sensitivity to discern this contribution near and below threshold, and spontaneous emission is negligible above threshold.

There are many types of Auger recombination that can occur in these structures. In this wavelength regime, Auger losses are dominated by processes in which an electron and hole recombine and the excess energy is used to excite another electron high into a conduction subband (CHCC process)[21], and depend strongly on details of the band structure. 

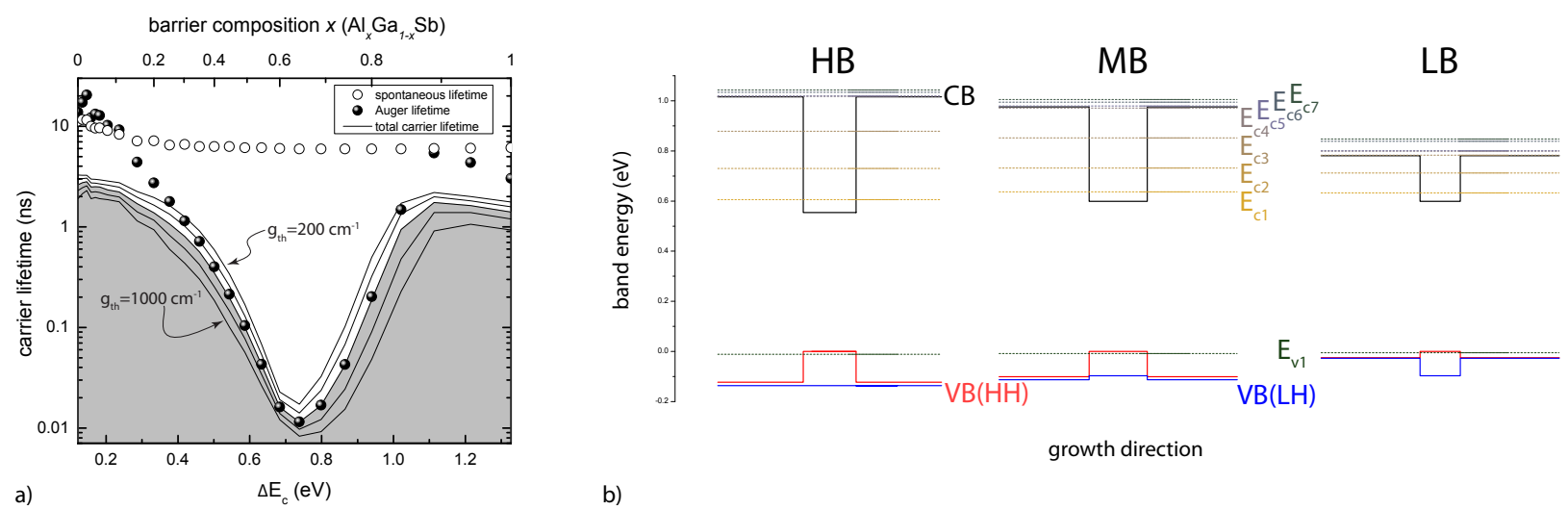

Figure 1. Carrier lifetime dependence on conduction band offset.

a) Contributions to room temperature carrier lifetime as a function of $\Delta E_{c}$. Spontaneous and Auger lifetimes are given for $g_{t h}=600 \mathrm{~cm}^{-1}$. Total carrier lifetimes (including an SRH lifetime of $4.5 \mathrm{~ns}$ ) are given for $g_{\text {th }}$ of 200,400,600,800, and $1000 \mathrm{~cm}^{-1}$. The total carrier lifetime associated with the spontaneous and Auger lifetimes plotted is emphasized with a shade underneath. b) transition energies between the lowest conduction band state and excited states. The drop of Auger lifetime in the region that $\Delta E_{c} \simeq E_{g}$ indicates the increased availability of states available for Auger transitions.

\subsection{Engineering Approaches to Auger Processes}

Generally, when varying the barrier potential, $\Delta E_{c}$, the dominant factor is the excess photon energy $\left(\sim E_{g}\right)$ with respect to the barrier level. Commonly, the approach is to design for a large $\Delta E_{c}$, improving carrier capture and reducing carrier density. The reduced carrier density is intended to improve the Auger lifetime through the empirical relation:

$$
C \equiv\left(\tau_{c} N_{t h}^{2}\right)^{-1},
$$

where $C$ is a proportionality constant known as the "Auger coefficient". The excited carrier transitions from a localized conduction band above the potential barrier to a bulk state.However, for a given wavelength, the Auger lifetime lifetime can vary dramatically, primarily depending on the $E_{g}$ relation to $\Delta E_{c}$.

This relation is summarized in Figure 1a, where we compute the lifetimes at $300 \mathrm{~K}$ holding the gain constant, adjusting carrier density to compensate varied differential gains. Carrier lifetime contributors (spontaneous and Auger lifetimes) are plotted as a function of barrier height in empty- and filled-symbols assuming a threshold of $600 \mathrm{~cm}^{-1}$, as will be seen to be a reasonable approximation for two of our devices. Assuming a SRH lifetime of $4.5 \mathrm{~ns}$ (also determined through fitting to experimental data, as shown in Section 3.5), the total carrier lifetime is also shown by solid black lines. These are calculated for $200 \mathrm{~cm}^{-1}-1000 \mathrm{~cm}^{-1}$, with the data corresponding to $600 \mathrm{~cm}^{-1}$ specifically identified through the grey fill beneath it. Because the Auger recombination is sensitive to subband positioning, and we are dominated by CHCC processes, conduction subband positions are plotted relative to the lowest electronic subband in Figure 1b. Finally, for reference, we plot the photon energy in Figure 1b, corresponding to the value of $E_{g}$ on this same scale to show where resonant transitions are found, causing increased Auger recombination wherein excess energy created from an electron/hole recombination are easily transferred from $E_{1}$ to an excited electron state in the conduction band $E_{x}$. 
In this wavelength regime, all practical structures are found in the region where $E_{g} \gg \Delta E_{c}$. At low values for $\Delta E_{c}$, the spontaneous emission lifetime (roughly proportional to inverse threshold carrier density), is long. Moreover, for Auger recombination in this region, the final electron is excited into states that are delocalized in the barrier, reasonably approximated by bulk-states, and a transition from a confined subband to this delocalized band requires a large transfer of momentum[22]. Because the Coulomb matrix elements that mediate the Auger transitions strongly decrease with a change in momentum, the Auger transition rate decreases (lifetime increases) with increasing excess energy above the barrier level.

At higher barriers $\left(\Delta E_{c} \gtrsim 0.35 \mathrm{eV}\right)$, Auger recombination dominates the carrier lifetime as the $E_{g} \simeq \Delta E_{c}$. This is due, in part to carrier excitations to more localized wavefunctions resulting in reduced momentum transfer. Within this region, coupling is very dependent on band-structure. Of note is the region where $E_{6}-E_{1}$ and $E_{7}-E_{1}$ are close to $E_{g}$, which promotes resonant transitions; the transition probability between these excited electronic states increases dramatically, causing the Auger lifetime to drop to around 10 ps. The effects can also be seen where $\Delta E_{c}>1.2 \mathrm{eV}$ in Figure 1 . In this region, $E_{5}-E_{1}$ is sufficiently resonant with $E_{g}$, reducing $\tau_{c}$ by a factor of 2 .

For values $x \gtrsim 0.45$ in $\mathrm{Al}_{x} \mathrm{Ga}_{1-x} \mathrm{Sb}$, the $X$ valley drops below the $\Gamma$ valley, causing the material to become an indirect bandgap[20]. This condition is generally avoided near the quantum-well, especially in electrically injected lasers, and where optically pumped lasers absorb significant pump in the barrier regime, typically to increase net absorption. However, we may computationally explore the region of strong electronic confinement, where the CHCC coupling is between two quantized states (where $E_{g}<\Delta E_{c}$ ), also addressed in Reference 22. In this case, the Auger lifetime is smallest when a subband is resonant with the lowest electronic subband, with a energy difference of $\sim E_{g}$, e.g. without requiring a transfer of in-plane momentum. As such, this relation is strongly dependent on the specifics of the structure, and generalities are significantly more difficult to make.

For comparison to the common $C N^{3}$ relation for the Auger recombination, Figure 2 shows the Auger lifetime (repeated from Figure 1) and how that relates to the analytical Auger coefficient $C$. The inverse relation dominates the general trend because $N_{t h}$ is relatively constant for most values of $\Delta E_{c}$. At very low values for $\Delta E_{c}, N_{t h}$ increases dramatically due to the poor carrier confinement, and this relation deviates significantly. We also show the approximation from Reference 22 for the CHCC coefficient for the case where $E_{g} \gg \Delta E_{c}$. It should be noted that the term $V_{c}$ is used in place of $\Delta E_{c}$ in Reference 22.

The three structures all represent a region where $E_{g}>\Delta E_{c}$, this ratio being 1.32, 1.62, and 3.35 for $\mathrm{HB}$, $\mathrm{MB}$, and LB, respectively. Only the LB structure is well approximated by the coupling from the discrete to the continuous spectrum in the conduction band[22].

\subsection{Growth and Fabrication}

\subsection{Method and Characterization}

An enabling tool for producing mid-infrared laser heterostructures as complicated as

$\mathrm{In}_{x} \mathrm{Ga}_{1-x} \mathrm{As}_{1-y} \mathrm{Sb}_{y} / \mathrm{GaSb}$ semiconductor lasers is molecular beam epitaxy (MBE), whereby monolayers of single crystals can be synthesized with atomic layer precision. A Gen-II molecular beam epitaxy reactor (in support of this research effort) was successfully transferred from Wright-Patterson Air Force Base (WPAFB) to University of Missouri, Columbia (UM). It was employed for the production of As-based quantum cascade 


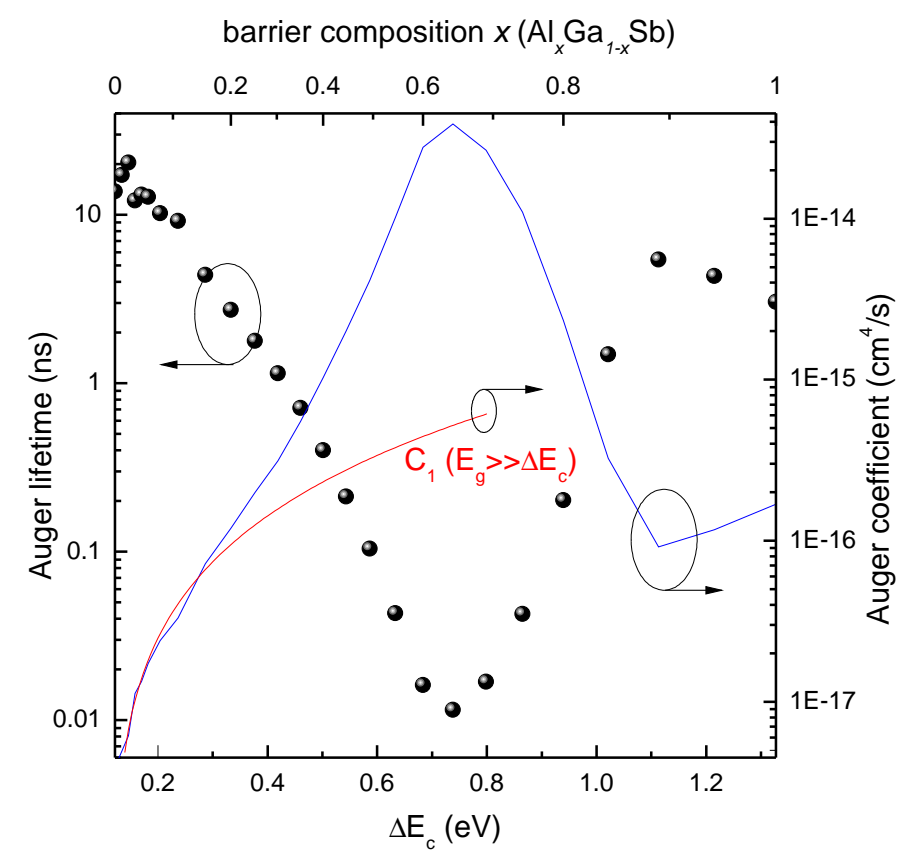

Figure 2. Auger lifetime and coefficients calculated at room temperature.

Comparison of calculated Auger lifetime vs. Auger coefficient, where the latter $(C)$ is taken to be equivalent to $\left(\tau_{c} N_{t h}^{2}\right)^{-1}$. $C_{1}$ for the CHCC process as given in Reference 22 .

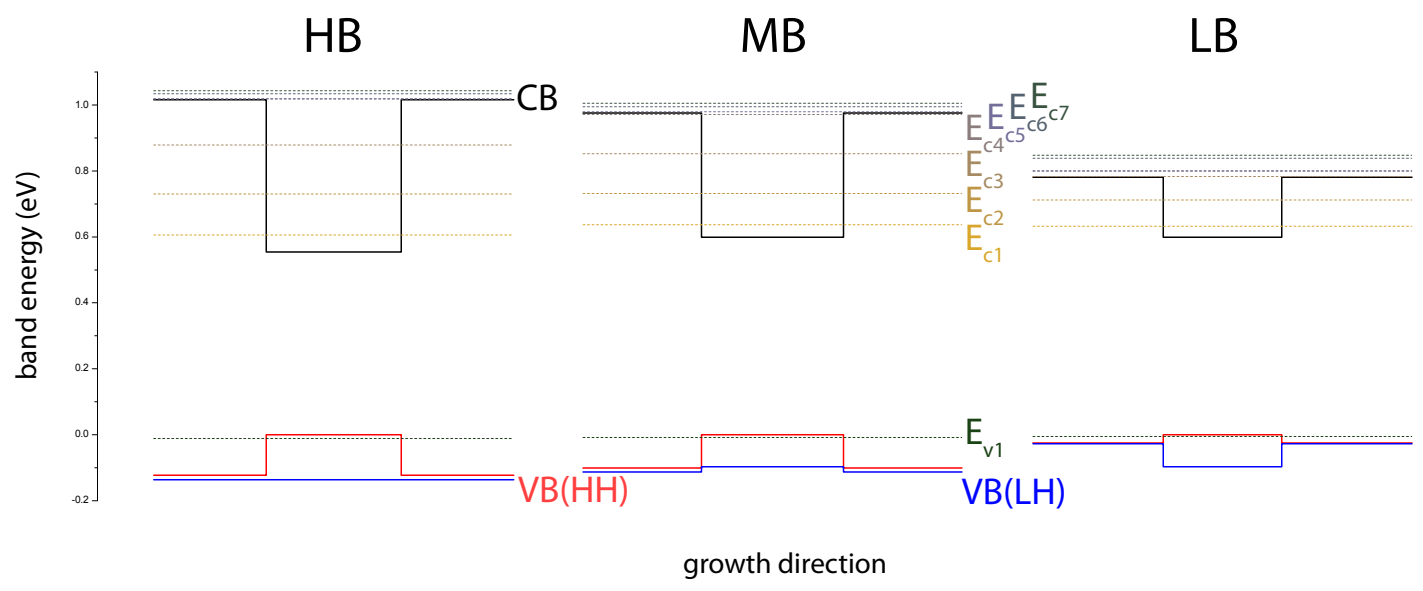

Figure 3. Energy diagrams of the three structures HB, MB, and LB structures.

Conduction band is marked " $C B$ ", the heavy-hole valence band is labeled " $V B(H H)$ ", while the light-hole valence band is marked " $V B(L H)$ ". The calculated electron energy bands $\left(E_{c n}\right)$ and hole energy bands $\left(E_{v n}\right)$ are also plotted for each structure. 


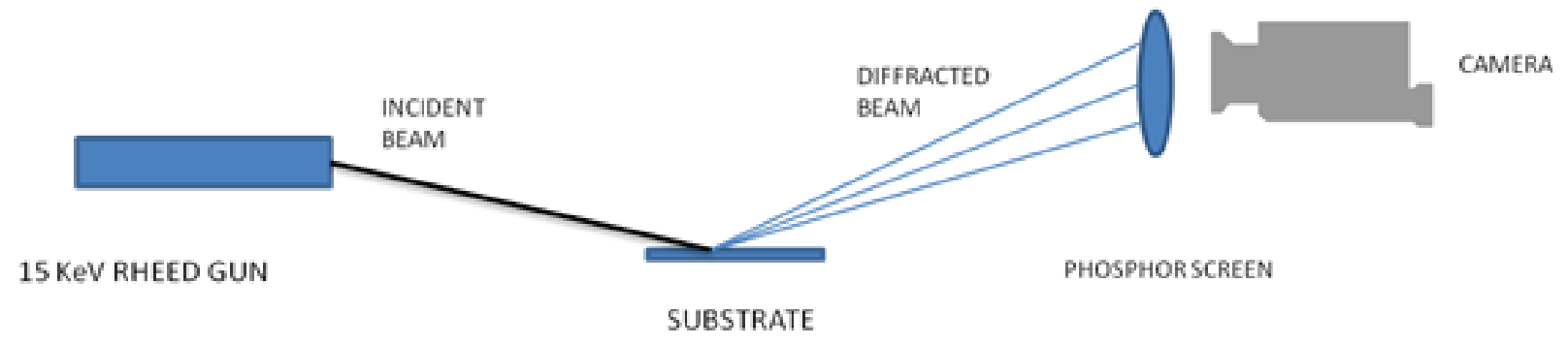

\section{Figure 4. RHEED schematic}

Staib Instruments RHEED system with a 12 bit digital camera and $K$-space 400 analytical system.

lasers and type-I vertical external cavity surface emitting laser (VECSEL) devices. Device structures were synthesized via molecular beam epitaxy (MBE) using detailed band structure engineering for the purpose of addressing optical power limitations in mid-infrared devices. Various VCSEL structures with various barrier heights were simulated, fabricated and analyzed.

$\mathrm{In}_{x} \mathrm{Ga}_{1-x} \mathrm{As}_{1-y} \mathrm{Sb}_{y} / \mathrm{GaSb}$ structures are produced using MBE, where several ovens of ultrapure materials were heated to produce an evaporated beam of molecules. The MBE process conditions necessary for producing high-quality devices include an ultra-high vacuum environment $\left(10^{-10}\right.$ Torr $)$ yielding material with impurity levels below ten parts per billion, precise control and reproducibility of composition and doping, and high-quality layers with very abrupt interfaces. At the facilities of one of our collaborators (University of Missouri), the MBE system consists of three separate, but connected vacuum chambers (i.e.a growth chamber, a buffer chamber and an intro chamber) with ion- and cryo-pumps to obtain requisite vacuum levels. The growth chamber also included a titanium sublimation pump (TSP), which was used to further enhance the vacuum environment.

The intro chamber is primarily for housing MBE-grown samples and for loading new substrates, while vacuum was maintained in the other two chambers via manual gate valves. A continuous supply of liquid nitrogen throughout the cryoshroud and substrate heater [along with university-supplied chilled water around the Knudsen cells (k-cells) and through bulk evaporator and cracker containing cells] are used to provide cooling, minimize outgassing of the vacuum chamber and minimize thermal contributions of adjacent cells. To maintain a specific process condition for each experiment, a cluster of proportional-integral-derivative (PID) closed loop control systems that monitored temperature zones within each oven are integrated along with computer controlled (pneumatic) shutters in front of each cell for precise control over the deposition rate. The MBE system contains arsenic, antimony, gallium, indium, aluminum, silicon (doping), GaTe (doping), and beryllium (doping).

For real-time, in-situ process monitoring, a Staib Instruments reflection high-energy electron diffraction (RHEED) system with a 6-in phosphor screen is used to analyze all device structures during growth. The use of RHEED to monitor the surface during changes in substrate temperatures, growth rates, and flux ratios is vital to the success of achieving good surface morphology. Apart from surface morphology characteristics, RHEED is also used in determining the substrate surface conditions. Figure 4 shows a typical setup and interaction of RHEED with the substrate surface and recorded using a camera. 
After loading, samples are transferred into the growth chamber and ready for growth after removal of the protective oxide layer. The laser structures in this research effort are grown on epi-ready (100) GaSb substrates from WaferTech. Beam equivalent pressures at possible growth temperatures for all k-cells cells are periodically monitoring using an ion gauge lowered directly in the path of where the substrate would be positioned during growth. These measurements are also used to calculate the activation energy of each k-cell. The RHEED patterns are used to monitor the surface morphology and growth kinetics at specific temperatures.

$\mathrm{In}_{x} \mathrm{Ga}_{1-x} \mathrm{As}_{1-y} \mathrm{Sb}_{y}$ remains an important semiconductor material for mid-infrared photonic devices. Conduction and valence band alignment of $\operatorname{In}_{x} \mathrm{Ga}_{1-x} \mathrm{As}_{1-y} \mathrm{Sb}_{y}$ quantum wells with other zinc-blende compounds (aluminum antimonide (AlSb), GaSb, indium arsenide (InAs)) provide advantageous performance enhancements to higher efficiency gain mediums in $\sim 3 \mu \mathrm{m}$ sources. Unfortunately, the ability to synthesize $\mathrm{In}_{x} \mathrm{Ga}_{1-x} \mathrm{As}_{1-y} \mathrm{Sb}_{y}$ pseudomorphically across the compositional range has been limited by substrate availability. Lattice mismatch with $\mathrm{GaSb}$ has prohibited the exploitation of the entire wavelength tuning range that $\operatorname{In}_{x} \mathrm{Ga}_{1-x} \mathrm{As}_{1-y} \mathrm{Sb}_{y}$ could potentially offer. There has been considerable effort to exploit high-indium content $\operatorname{In}_{x} \mathrm{Ga}_{1-x} \mathrm{As}_{1-y} \mathrm{Sb}_{y}$ for longer wavelength operation, yet high misfit dislocation densities are inevitable and the miscibility gap is a formidable barrier (see Figure 5b). To date, the ability to cover the compositional range of $\operatorname{In}_{x} \mathrm{Ga}_{1-x} \mathrm{As} 1-y \mathrm{Sb}_{y}$ as well as its range of properties remains unresolved. In this effort, we explore $\operatorname{In}_{x} \mathrm{Ga}_{1-x} \mathrm{As}_{1-y} \mathrm{Sb}_{y} / \mathrm{GaSb}$ structures, where the measured indium mole fraction $(x)$ varies from $x=0$ to $\mathrm{x}>0.50$. In addition to atomically smooth structures with growth two-dimensional growth fronts Figure 5a, we observed three-dimensional networks of quantum dashes and results reveal a self-organized composition modulation. Some physical features of the quantum dashes include near one-micron lengths, 90-deg flip in orientation, and uniformity across a $20 \times 20 \mu \mathrm{m}$ area. Samples of MBE-grown mid-infrared VCSEL structures under various process conditions are presented in Figure 6 to demonstrate the influence on the process conditions on wavelength tunability. While these devices demonstrate lower indium concentration than planned for the MWIR structures, the principals are identical and we're using the concepts transitioning to MWIR as well.

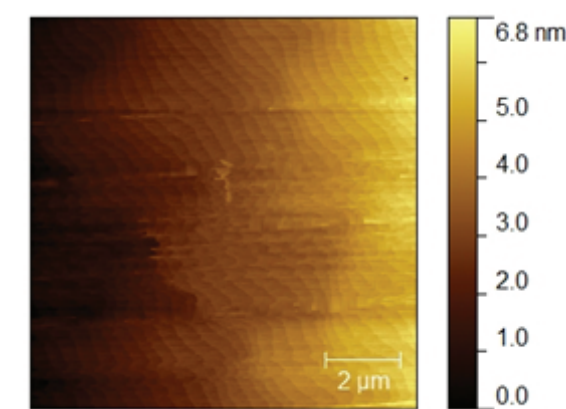

a)

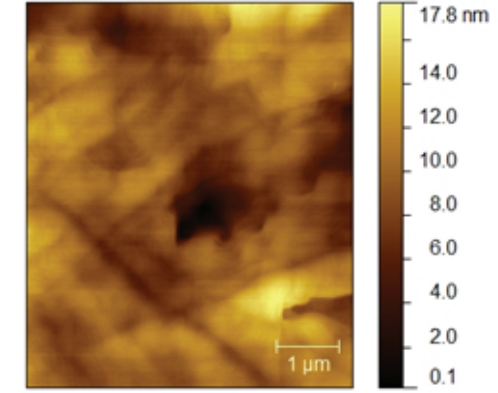

b)

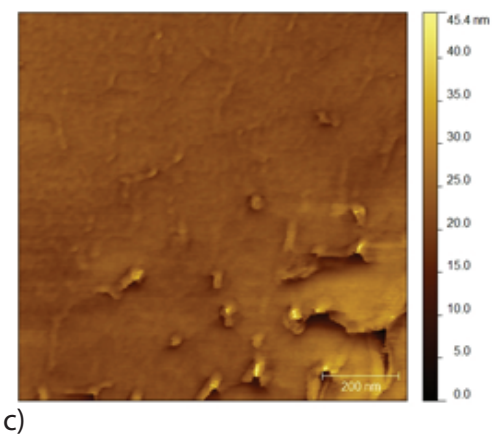

Figure 5. Planview AFM of InGaAsSb surface with varying concentrations a) $\left.\left.I n_{0.5} G a_{0.5} S b, b\right) I n_{0.9} G a_{0.1} S b, c\right) I n_{0.5} G a_{0.5} A s_{0.2} S b_{0.8}$ 


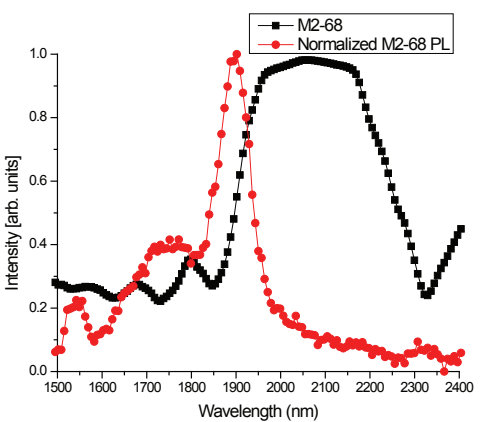

a)

\section{Figure 6. Reflectivity and photoluminescence of several samples}

a) InGaSb VECSEL structure (with DBR), b) InGaSb VECSEL structure with additional DBRs and modified growth conditions, and c) InGaSb VECSEL structure (modified growth conditions).

\subsubsection{Test Structures}

Prior to laser growth and to ensure precise alloy composition and thickness for each laser design presented, deposition rates are varied, measured in situ by RHEED, and confirmed post-growth using HR-XRD. Samples are also analyzed by atomic force microscopy (AFM), which demonstrates that these structures were indeed produced via step flow growth.

$\mathrm{In}_{x} \mathrm{Ga}_{1-x} \mathrm{Sb}$ structures are also produced to calibrate indium composition in the quantum well and to ensure $2 \mu \mathrm{m}$ emission, which is confirmed with photoluminescence. For each laser design, the MBE process conditions (flux ratio, substrate temperature, and deposition rate) are selected to ensure quality and composition of each binary and ternary layer. The shutter sequence and antimony valve-positioner are computer controlled, thus maintaining run-to-run consistency. Each quantum well/barrier combination is embedded in an $\mathrm{AlGaSb}$ waveguide that is adjusted to maintain a pump absorption of approximately $85 \%$, and a transverse mode overlap of about 5\%. In order to accomplish this, HB and MB structures have 3 repeats of the quantum wells, each separated by $100 \mathrm{~nm}$ to assure electronic isolation. To achieve the same overlap and absorption, while maintaining single transverse mode operation, the LB structure necessitates only two quantum wells.

Strained $\mathrm{In}_{x} \mathrm{Ga}_{1-x} \mathrm{Sb} / \mathrm{Al}_{y} \mathrm{Ga}_{1-y} \mathrm{Sb}$ quantum well lasers are produced by solid-source MBE system. Semi-insulating GaSb substrates are mounted in indium-free molybdenum blocks with diffuser plates (located between the substrate and the substrate heater) to achieve improved temperature uniformity across the wafer. During substrate outgassing, deoxidation temperatures are monitored using a $10 \mathrm{keV}$ RHEED system and are confirmed by a " $1 \times 3$ " reconstruction pattern. The growth temperatures are relative to the GaSb deoxidation temperature and also referenced by the " $1 \times 5$ " phase transition while under antimony over-pressure.

Each quantum well/barrier combination is embedded in an $\mathrm{AlGaSb}$ waveguide that is adjusted to maintain a pump absorption of approximately $85 \%$, and a transverse mode overlap of about $5 \%$. In order to accomplish this, HB and MB structures have 3 repeats of the quantum wells, each separated by $100 \mathrm{~nm}$ to assure electronic isolation. To achieve the same overlap and absorption, while maintaining single transverse mode operation, the LB structure necessitates only two quantum wells. 


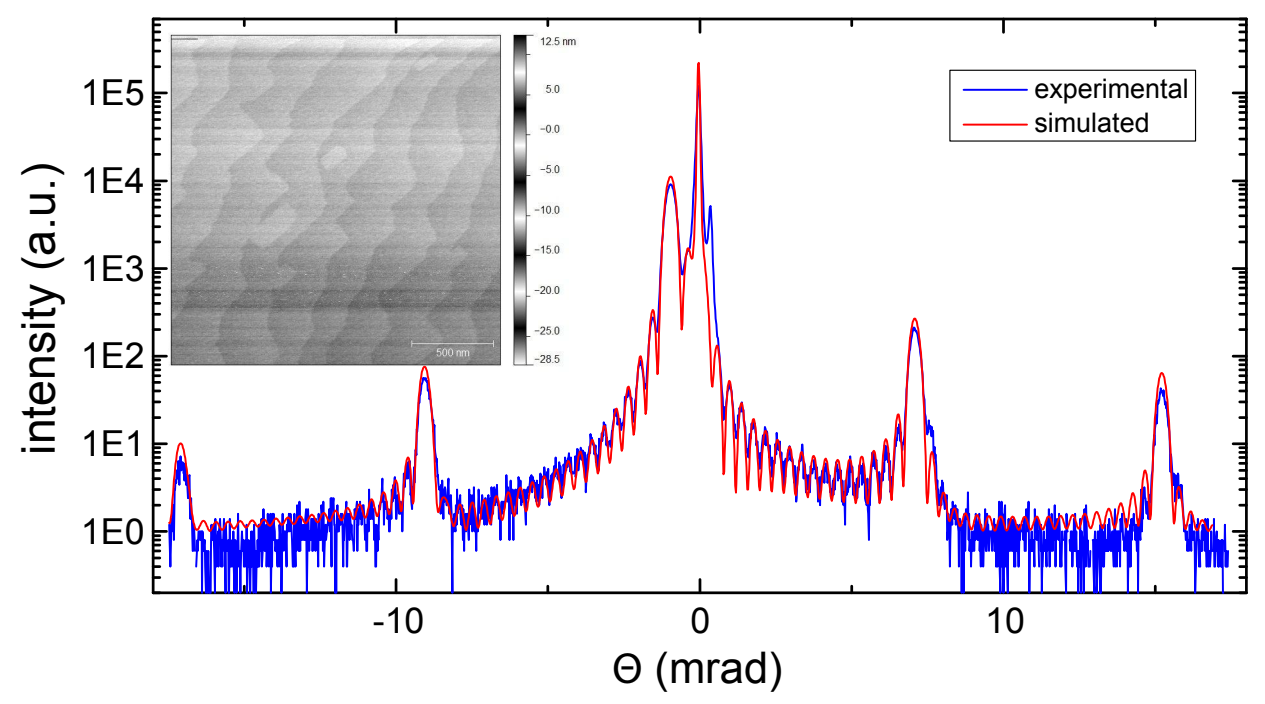

Figure 7. Example of HR-XRD $\Omega-2 \Theta$ rocking curves.

This data represents AlSb/GaSb superlattices along with calculated diffraction pattern, representing faithful reproduction material composition and layer thickness. Inset is as example surface profile measurement by AFM.

As the gain-guided structures are defined by the optical pump, no lithographic steps are necessary. The bare wafers are thinned to nominally $80 \mu \mathrm{m}$ using mechanical lapping and then cleaved into bars of various lengths $(375 \mu \mathrm{m}$ to $1500 \mu \mathrm{m})$. In this report, only $1500 \mu \mathrm{m}$ length cavities are used.

\subsection{Type-I Results and Discussion}

Laser bars are mounted epitaxial-side up using thermal paste to a low-oxygen copper heat sink temperature controlled using a two-stage thermoelectric cooler, allowing various temperatures at, and around room temperature (from about $265 \mathrm{~K}$ to nominally $325 \mathrm{~K}$ ). We maintain a slow dry nitrogen purge to avoid condensation at temperatures below the dew-point. The lasers are pumped using a $980 \mathrm{~nm}$ semiconductor laser bar with a spot $125 \mu \mathrm{m}$ wide and $3 \mathrm{~mm}$ long. Pulses are $2 \mu$ s long with a $1 \mathrm{kHz}$ pulse repetition frequency, avoiding thermal loading.

A fraction of the optical power is collected using a fiber-coupled extended-range InGaAs detector. Both the pump laser and device powers are time-gated to remove pulse transients. The pump signal is calibrated and compared to $\mathrm{CW}$ operation, integrating over multiple cycles, to determine the appropriate power vs. detector voltage. While the device power remains uncalibrated, lasing threshold is the most important metric to evaluate carrier losses and therefore device powers remain in arbitrary units, although the noise floor of this detector is $\sim 0.01$ on this arbitrary scale. Finally, we also measure spectra at each temperature and pump level to confirm the device is behaving as we expect.

We measure the power characteristics from approximately 350 lasers spanning the three growth designs mentioned in Section 3.1, and report these in Figure 8. The mean data from each of the three structures are indicated by the data points, while the error bars signify standard deviation from the collected data. 


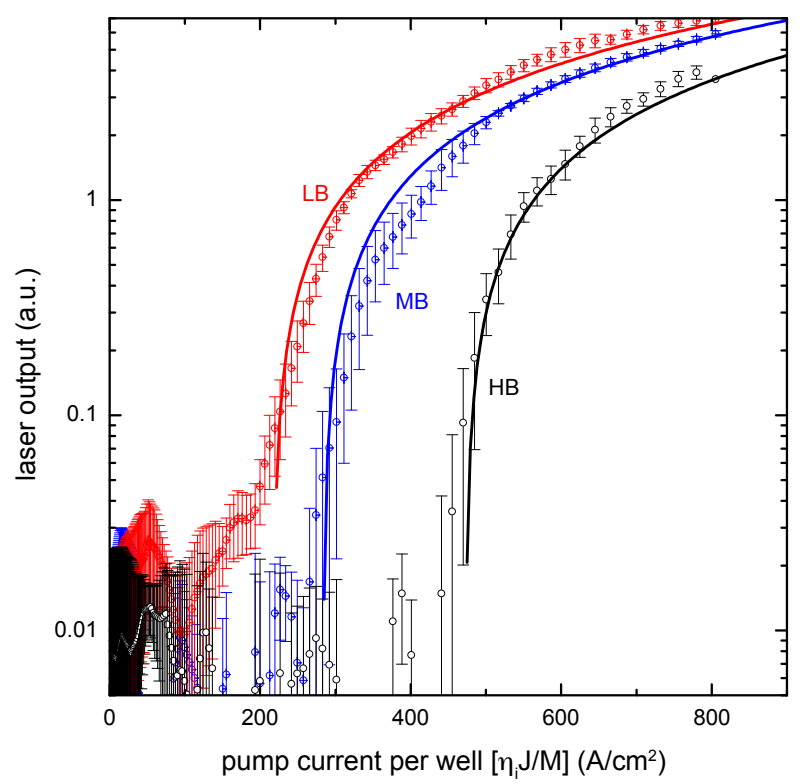

Figure 8. Laser power characteristics comparing devices with various barrier heights. Laser power characteristics given for the three designs outlined in Section 3.1, as a function of equivalent pump current density. Data is shown on a logarithmic plot to emphasize the threshold.

Although we pulse the pump to avoid thermal loading, we ignore transients allowing us to set the left sides of Equations 1 and 2 to zero to find the steady state. In this case, we find the threshold condition of the laser:

$$
\frac{\eta_{i} J}{M}=\frac{N}{\tau} \equiv \frac{N}{\tau_{a}}+\frac{N}{\tau_{b}}+\frac{N}{\tau_{c}},
$$

where $M$ is the number of wells, and $\tau$ represents the combination of all three carrier loss terms, and is commonly referred to as the "carrier lifetime." Figure 8 shows the power characteristics of this laser. The absorbed pump fluence per well is equivalent to $\eta_{i} J \hbar \omega_{p} / M$ in Equation 1, and is, of course, the driving term of the laser. It is immediately evident that the HB structure has the highest threshold per well, a result that is not unpredictable due to the large Auger recombination as discussed in Section 3.1 and calculated in Figure 1.

From Figure 8, we see the threshold per well is 480, 283, and $255 \mathrm{~A} / \mathrm{cm}^{2} /$ well, which corresponds to equivalent pump fluences of 606,358 and $255 \mathrm{~W} / \mathrm{cm}^{2} /$ well for the $\mathrm{HB}, \mathrm{MB}$, and LB structures, respectively. Because $\eta_{i}$ is used as a fit parameter, we only find relative changes to the carrier lifetimes. Experimental results compare favorably to theory in the MB and HB structures for an internal absorption $\alpha_{i}=10 \mathrm{~cm}^{-1}$ and a SRH lifetime $\tau_{a}=4.5 \mathrm{~ns}$. In contrast, the LB structure had a larger internal loss of $\left\langle\alpha_{i}\right\rangle=20 \mathrm{~cm}^{-1}$, but the SRH lifetime was larger as well (20 ns), offsetting some of this additional loss. This is not altogether surprising because the LB structure, with only two wells, contains fewer interfaces within the high carrier density region, where SRH recombination typically presents itself. However, the transverse mode profile contains longer tails and therefore could incur higher losses due to optical scattering.

From these parameters, we can determine the threshold carrier densities $\left(N_{t h}\right)$ are $1.15 \times 10^{12} \mathrm{~cm}^{-2}$, $1.67 \times 10^{12} \mathrm{~cm}^{-2}$, and $2.12 \times 10^{12} \mathrm{~cm}^{-2}$ for the $\mathrm{HB}, \mathrm{MB}$, and LB structures, respectively. From these, we can compute the carrier lifetimes for the MB and LB structures as $2.46 \times$ and $6.56 \times$ that of the HB "standard" 


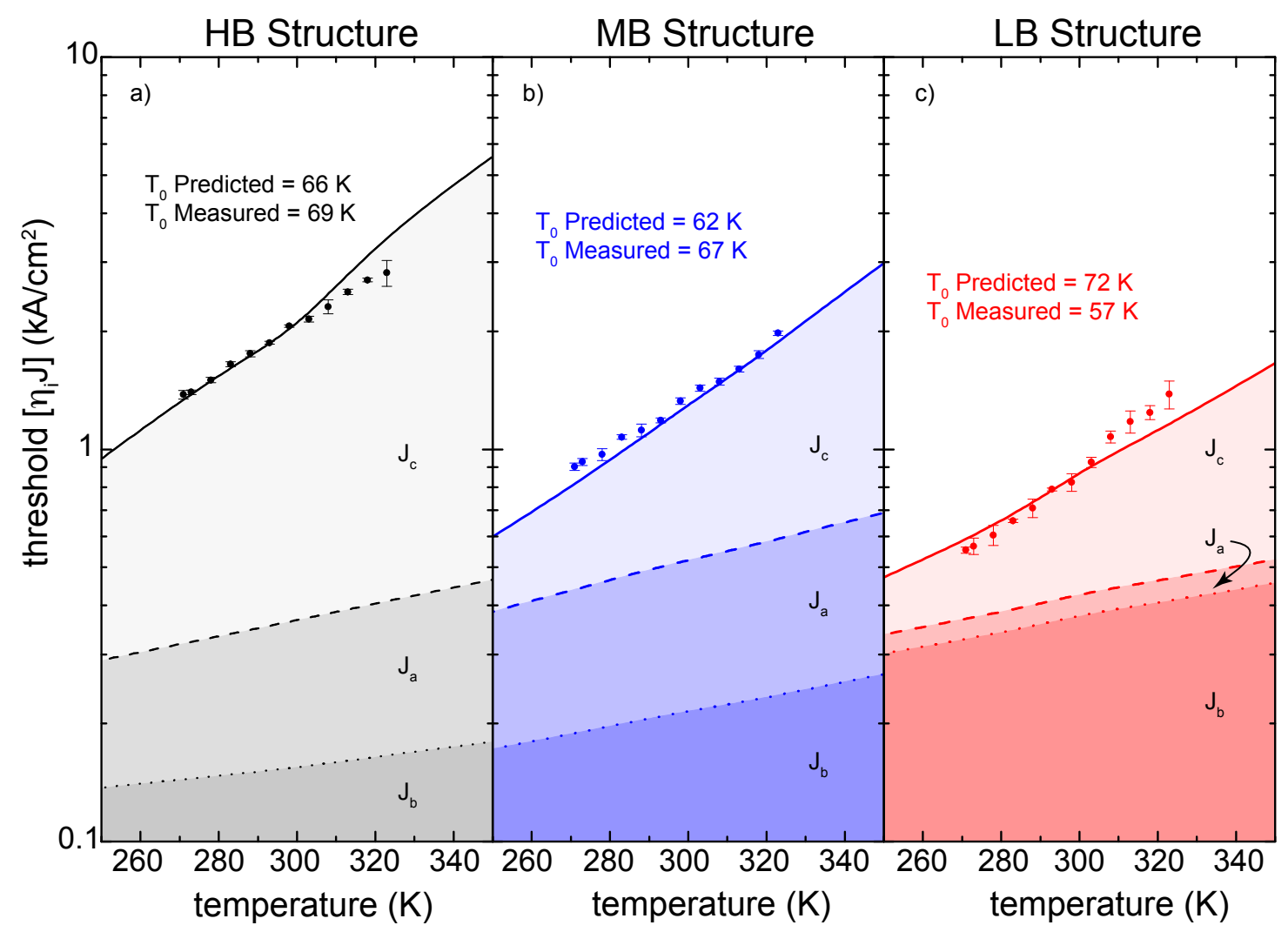

Figure 9. Measured and calculated threshold for various temperatures.

Threshold (in equivalent current) and $T_{0}$ and contributions from the three carrier loss contributors $J_{a, b, c}$ for a) the HB structure, $b$ ) the MB structure, and c) the LB structure.

structure. This represents a significant $(>6 \times)$ improvement of the carrier lifetime, resulting in a significantly lower threshold density.

A more definitive test of the model and the device improvement is the temperature dependance of the threshold, and therefore loss mechanisms. This dependence is commonly denoted as $T_{0}$ and empirically behaves as an exponential growth[21]. Although this relation is not completely accurate[19], it is often a suitable fit for a wide variety of temperatures.

Figure 9 shows the temperature-dependance of the HB (Figure 9a), MB (Figure 9b), and LB (Figure 9c) structures. These curves, while including the $\eta_{i}$ term, are not normalized by the number of wells as in Figure 8. We also show in Figure 9 the contributing terms to the total threshold fluence. In each case, the relative contribution of the SRH, spontaneous, and Auger are denoted by $J_{a, b, c}$, respectively, and are computed as a function of temperature. The same terms for $\left\langle\alpha_{i}\right\rangle$ and $\tau_{a}$ are used for this graph as those used to compare in Figure 8.

We can see in each of these examples, the measured $T_{0}$ is closely predicted by the model. In the best case, these deviated by $3 \mathrm{~K}$ for the HB structure. We expect the kink at about $300 \mathrm{~K}$ should have been more evident. This kink is due to increased Coulomb matrix elements as $E_{g}$ drops at higher temperatures approaching $\Delta E_{c}$, where less momentum is required for the transition. In the worst case, this deviation was as high as $15 \mathrm{~K}$ for the LB structure, although this effect could be attributed to carrier transport (e.g.thermionic emission). 
For each of these cases, we can compare the fraction of Auger recombination to that of the standard HB structure. Indeed, despite the increased threshold carrier density, $J_{c}$ is reduced from $1.13 \mathrm{kA} / \mathrm{cm}^{2}$ to $0.25 \mathrm{kA} / \mathrm{cm}^{2}$, a 4-fold decrease in carrier losses due to Auger recombination. However, due to the threshold carrier density disparity, this is actually a $5.7 \times$ increase in Auger recombination lifetime, reflecting a significant improvement to Auger losses.

As stated previously, the metric is the quasi-empirical Auger coefficient $(C)$, related to $\tau_{c}$ by Equation 3 . Although this relation is not strictly held, we can calculate the value of $C$ relative to the HB structure for reference; this value is found to be $6.4 \times$ lower for the MB structure, and a significant $19.2 \times$ lower for the LB structure. We show then that $C$, most often considered constant for a given bandgap, to be easily varied by $20 \times$, and we can widen this more dramatically with stronger electron confinement (as high as $\sim 0.55 \mathrm{eV}$ with barriers composed of $\mathrm{Al}_{0.45} \mathrm{Ga}_{0.55} \mathrm{Sb}$ ), although this would not improve the device any more, but rather make the lower performing devices less suitable.

We therefore show this concept for directly engineering the Auger recombination rate is supported by experimental results. This approach and methodology is not only appropriate at $2 \mu \mathrm{m}$, but can be expanded to longer wavelengths. Such a numerical study is outlined in Section 3.7.

\subsection{IMF-grown Materials}

One of the promising techniques for MWIR lasers was the use of interfacial misfit (IMF) to grow III-Sb low-energy active regions on III-As high thermal conductive DBRs. Fig. 1(a) shows a schematic illustration of the fabricated VECSEL structure on a gallium arsenide (GaAs) substrate. The bottom DBR is comprised of 25 pairs of $\mathrm{Al}_{0.95} \mathrm{Ga}_{0.05} \mathrm{As} / \mathrm{GaAs}$. The active-region consists of $9 \mathrm{In}_{0.2} \mathrm{Ga}_{0.8} \mathrm{Sb} \mathrm{QW}$ separated by pump absorbing $\mathrm{Al}_{0.25} \mathrm{Ga}_{0.75} \mathrm{Sb}$ barriers. An AlSb confinement layer on each side of the active region ensures carrier confinement in the structure, and a thin GaSb capping layer is added on top to prevent oxidation. A low-resolution TEM image of the structure is shown in Figure 10a, the structure matches the epitaxially grown structure (only a few DBR-pairs are captured in image). Figure $10 \mathrm{~b}$ also shows the $\vec{E}$-field profile for the lattice-matched VECSEL. The key feature of the antimonide VECSEL based on the GaAs/AIGaAs DBRs is the ability to achieve lasing despite the $7.78 \%$ mismatched interface between the antimonide active region and the arsenide based DBR. The first quantum well is located within $500 \mathrm{~nm}$ of this highly mismatched interface. Thus for such a VECSEL to work the growth has to be optimized to achieve minimal threading dislocations in the active region and to achieve a reproducible relaxation of the metamorphic buffer.

\subsubsection{Limitations of the IMF for SWIR active regions}

Unfortunately, what was found was that when compared to the same active region on lattice-matched GaSb DBR, the structure grown on IMF had a much higher threshold power density than that of the lattice-matched VECSEL (nominally $13 \times$ higher) and the slope efficiency is approximately $1 / 4$ that of the structure grown on III-Sb. It can be speculated that the deterioration in the performance of the VECSEL when grown mismatched on GaAs compared to when it is grown lattice matched on GaSb can be attributed to the presence of threading dislocations in the active region when it is grown on the GaAs/AlGaAs DBR. In Equation 1, the second term on the right-hand side, taken alone, represents the resulting decay response when 


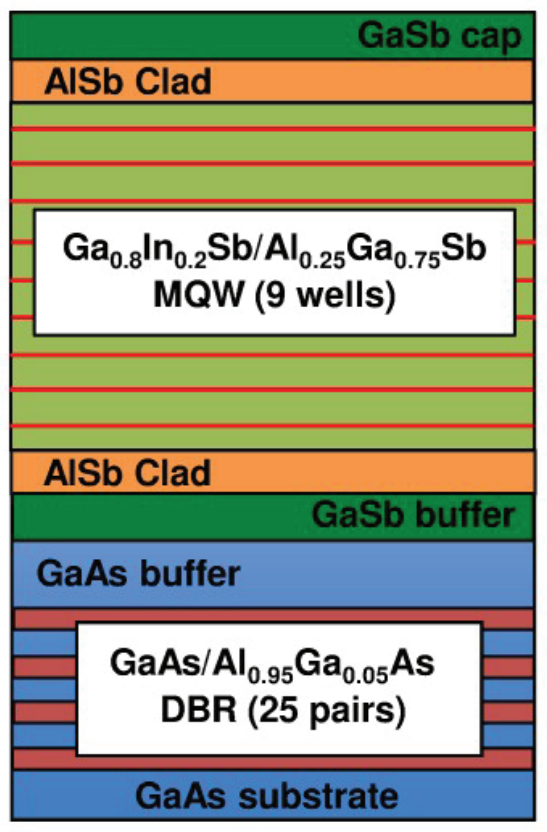

(a)

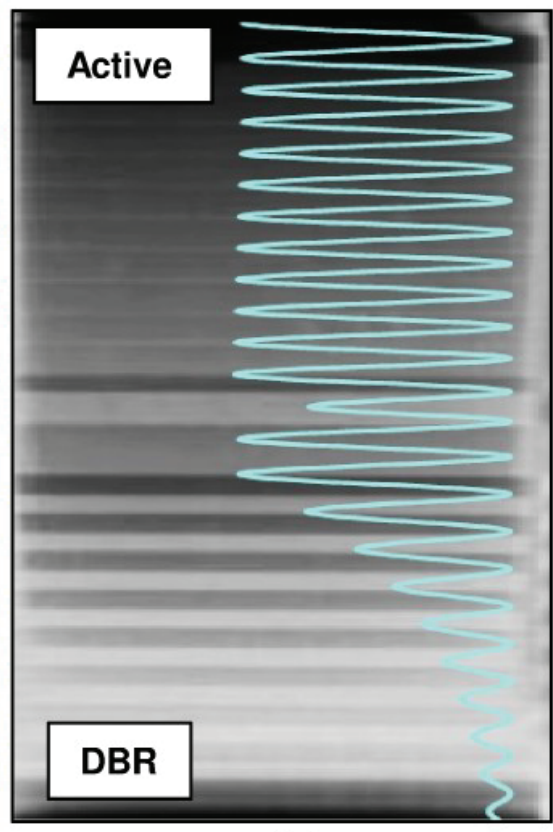

(b)

Figure 10. Layer structure and image of IMF structure

a) Schematic, b) cross-section TEM image and plot of $|E|^{2}$-field for lattice-mismatched $2 \mu \mathrm{m}$ III-Sb VECSEL grown on GaAs/AlGaAs DBR.

carriers are excited. If the lifetimes $\left(\tau_{a, b, c}\right)$ are constant, an exponential results ${ }^{1}$. Through the spontaneous emission term of the form $\beta_{s p} \Gamma \tau_{b}$ (where $\beta_{s p}$ is the rate at which spontaneous photons are coupled to the optical cavity modes, and $\Gamma$ is the modal overlap), this carrier decay is imprinted on the photon generation. As is custom in such laser systems, this term is neglected in Equation 2, an equation which is appropriate only above threshold where the spontaneous emission contributes insignificantly to the photon generation.

It is a reasonable to approximate the carrier decay terms by observing the time-resolved photoluminescence signal. Figure 11a shows several examples of the spectrally integrated PL signal as a function of time, after excitation time (occurring at approximately $2 \mathrm{~ns}$ ). The two sets of data are displayed for the average excitation power densities of 30 and $3000 \mathrm{~W} / \mathrm{cm}^{2}$. The measured transient photolumiscence (PL) intensities were generally well fitted with the double exponential function of $I(t)=I_{\text {dark }}+A_{1} e^{-t / \tau_{1}}+A_{2} e^{-t / \tau_{2}}$, where $I_{\text {dark }}$ is the dark counts without the pulsed laser excitation, $A_{1}$ and $A_{2}$ are the amplitudes of each term, and $\tau_{1}$ and $\tau_{2}$ are the decay time constants. These parameters were determined by the fittings shown by the solid lines in Figure 11a and are summarized in Table 1.

To identify the origin of the two decay time constants of $\tau_{1}$ and $\tau_{2}$, we have studied the time decay by spectral integrations in limited ranges. For example, the PL decay measured on the quantum well with IMF was spectrally separated into the two regions of 1367-1405 nm and 1406-1444 nm and integrated in each region. This spectral separation resulted in single exponential decays and the decay time constants determined in the lower and higher photon energy regions very nicely reproduced the $\tau_{1}$ and $\tau_{2}$ values given

\footnotetext{
${ }^{1}$ As discussed previously, these carrier lifetime contributions are considered constant only in the small carrier density modulation scheme - that is, they, themselves, are carrier density dependent, causing a "multi-exponential" decay. This complex decay can aid in the distinction between multiple non-radiative effects.
} 


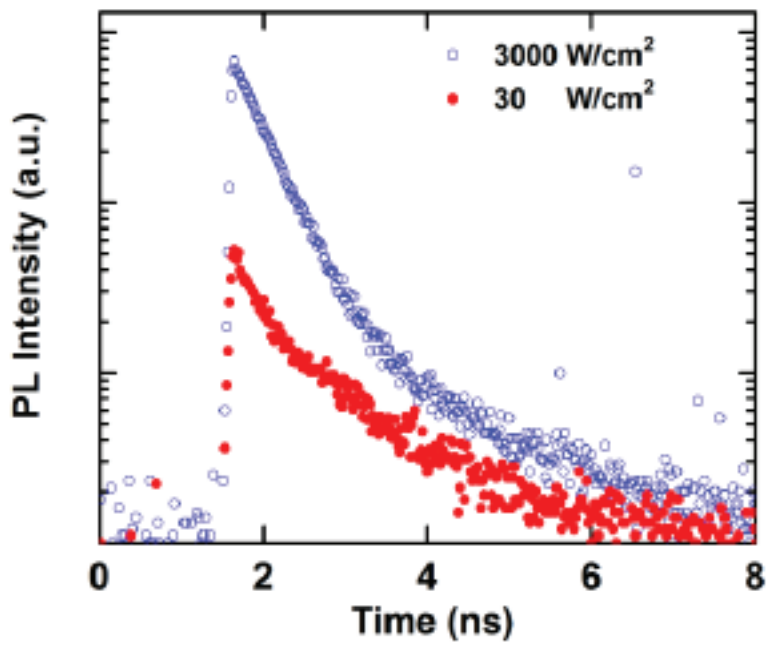

a)

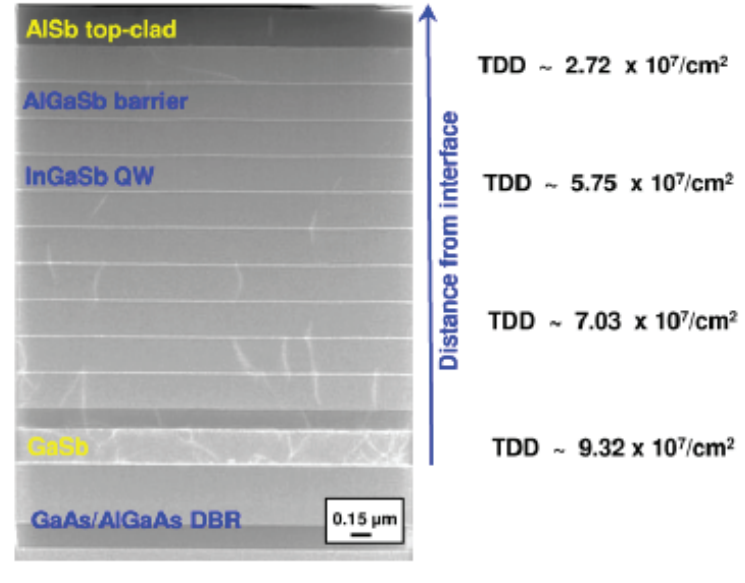

b)

Figure 11. Time-resolved photoluminescence and STEM images of structure

a) Time-resolved photoluminescence measured on $Q W$ grown with IMF with the average excitation poiwers of $30 \mathrm{~W} / \mathrm{cm}^{2}$ and $3000 \mathrm{~W} / \mathrm{cm}^{2}$, b) STEM image of lattice mismatched III-Sb VECSEL on GaAs/AlGaAs showing the source of the threading dislocations at the IMF array and subsequent reduction in the density as a function of distance from the mismatched interface

Table 1. IMF PL Decay Characterization

Time-resolved photoluminescence characterization

\begin{tabular}{c|ccc|ccc}
\hline \hline Excitation & \multicolumn{3}{|c}{ QW with IMF } & \multicolumn{3}{c}{ QW without IMF } \\
${\mathrm{W} / \mathrm{cm}^{2}}^{2}$ & $A_{1} / A_{2}$ & $\tau_{1}(\mathrm{ps})$ & $\tau_{2}(\mathrm{ps})$ & $A_{1} / A_{2}$ & $\tau_{1}(\mathrm{ps})$ & $\tau_{2}(\mathrm{ps})$ \\
\hline 30 & 4.47 & 1006 & 222 & 16.78 & 1560 & 250 \\
150 & 3.46 & 1000 & 260 & 6.44 & 1530 & 243 \\
750 & 3.55 & 1050 & 310 & 6.23 & 1450 & 248 \\
1550 & 2.98 & 1004 & 330 & 6.79 & 1550 & 258 \\
3000 & 2.69 & 1019 & 351 & 6.58 & 1590 & 257 \\
\hline \hline
\end{tabular}


in Table 1, respectively. Therefore $\tau_{1}$ and $\tau_{2}$ are the decay time constants related to the quantum well ground states and the excited states, respectively. The comparison of the two sets of data in Table 1 on the quantum wells with and without IMF shows a clear difference of the excitation power dependence of the $\tau_{2}$ value. With IMF the $\tau_{2}$ value and the amplitude $A_{2}$ increased with the excitation power, while they were independent of the excitation power without IMF. After the pulsed laser excitation of the quantum well samples, the excited electrons and holes experience energy relaxation. Therefore the $\tau_{2}$ value of the excited states includes this energy relaxation process and is much shorter than the $\tau_{1}$ value of the ground states. The increase of the excitation power generally enhances the band filling of the quantum well ground states with the photo-generated carriers, and this reduces the energy relaxation rate in both the conduction and valence bands due to the filling of the energy states above the band edges. This will result in the increase of the $\tau_{2}$ value for the quantum wells with IMF. The enhanced steep decay in Figure 11a for the higher excitation corresponds to the increase of the amplitude $A_{2}$. The fact that the quantum wells without IMF did not show any excitation power dependence indicates the presence of residual defects that prevent the band filling effect in the quantum well ground states.

This band filling effect is consistent with threading dislocations originating from the IMF. Figure 11b shows an transmission electron microscopy (TEM) micrograph of the III-Sb VECSEL that demonstrates a significant density of dislocations, which is reduced farther away from the IMF interface (at the $\mathrm{GaSb} / \mathrm{GaAs}$ junction). Carrier lifetimes due to defect recombination can be approximated via techniques described more in depth in References 23 and 24:

$$
\frac{1}{\tau_{\text {dislocation }}}=\frac{\mu k T}{q} \frac{l}{V}
$$

where $\mu$ is the carrier mobility, $q$ is the electron charge, $l$ is the projected dislocation length (estimate), $V$ is the volume being analyzed. In this way, we estimate the dislocation density $N_{D}$ as a function of distance from the $\mathrm{GaSb} / \mathrm{GaAs}$ interface (this information is presented in Figure 11b). The dislocation density reduces from $\sim 10^{8} \mathrm{~cm}^{-2}$ to about $10^{7} \mathrm{~cm}^{-2}$ at a distance of $3 \mu \mathrm{m}$ from the interface. Thus it is evident from the relationship between carrier lifetime and dislocation density that the higher threading dislocation density found in the III-Sb VECSELs grown lattice mismatched on GaAs/AlGaAs DBRs is detrimental to the VECSEL performance. Furthermore this high threading dislocation density in the III-Sb on GaAs/AlGaAs can also be linked to the high threshold density, lower efficiency and reduced maximum output power compared to a lattice matched III-Sb VECSEL.

\subsection{Longer wavelengths}

Following on our success with devices operating at $\lambda \sim 2 \mu \mathrm{m}[25,26]$, we have proceeded to structures designed to operate at $3.5 \mu \mathrm{m}$, which is much longer than type-I lasers are typically shown to operate. In order to do this, we must rely on quaternery material (InGaAsSb quantum wells), and compensate the strain with quaternary barriers ( $\mathrm{AlGaAsSb}$ ). Figure 2 shows a device that is nominally similar to Reference 14, where the $14 \mathrm{~nm}$ wide quantum well is made of $\mathrm{In}_{0.5} \mathrm{Ga}_{0.5} \mathrm{As}_{0.19} \mathrm{Sb}_{0.81}$, flanked by $\mathrm{Al}_{x} \mathrm{Ga}_{1-x} \mathrm{As}_{y} \mathrm{Sb}_{1-y}$ barriers. In Figure 2a, the aluminum concentration in the barrier material is altered to adjust the $\Delta E_{c}$, while the arsenic is adjusted to maintain a constant strain throughout the simulated structures.

All practical devices for such a structure are found for $0.4 \lesssim \Delta E_{c} \lesssim 0.82 \mathrm{eV}$. The lower bounds $(\sim 0.4 \mathrm{eV})$ is where barrier material is GaSb. At the upper bounds (for values $x \gtrsim 0.45$ in $\mathrm{Al}_{x} \mathrm{Ga}_{1-x} \mathrm{AsSb}$ ), the $X$ valley 

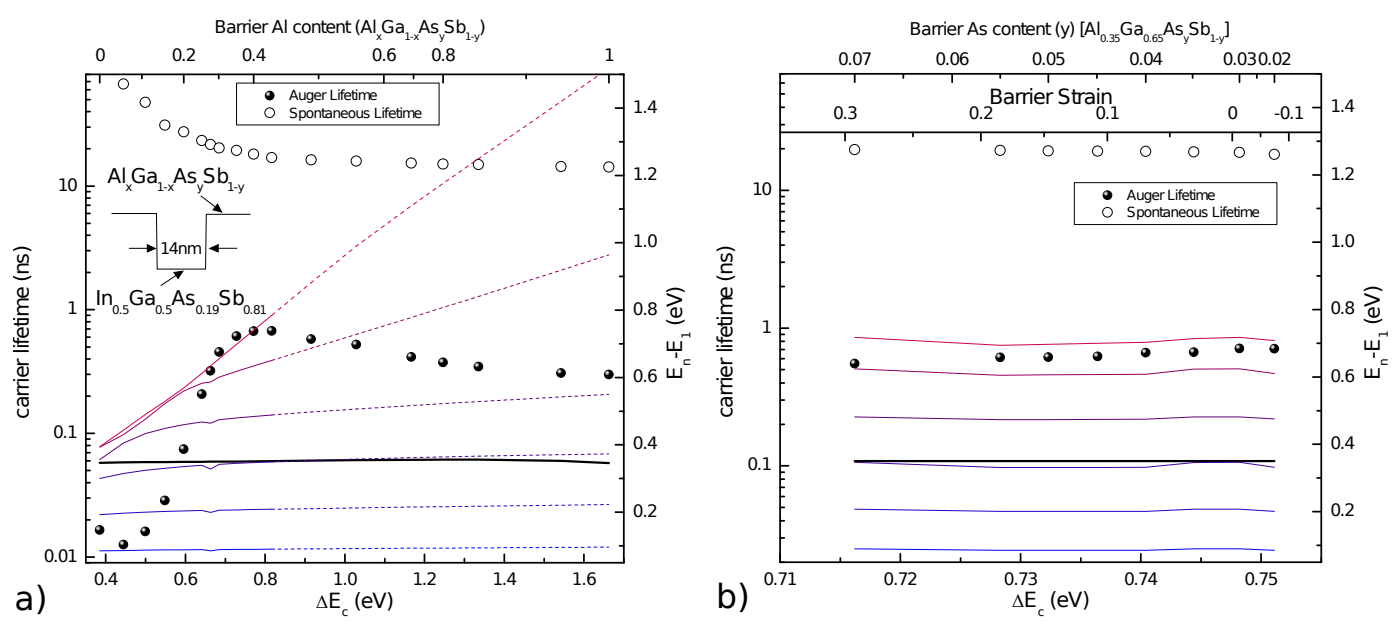

\section{Figure 12. Simulated Auger and spontaneous lifetimes for $\sim 3.5 \mu \mathrm{m}$ designed quantum wells.}

a) Varied $\Delta E_{c}$ and constant strain, and b) at the largest Auger lifetime of (a), varying strain through arsenic content.

drops below the $\Gamma$ valley, causing the material to become an indirect bandgap[20]. This condition is generally avoided near the quantum-well, especially in electrically injected lasers, and where optically pumped lasers absorb significant pump in the barrier regime, typically to increase net absorption. There is a significant inefficiency in this regime due (known as the "Richardson Factor") to electron momentum mismatch upon transition from the barrier to the well region.

The devices numerically investigated and reported in Figure 12 is in a distinctly different regime than that previously reported[25], in that the final state in the CHCC Auger process is confined within the quantum well. In this region, Within this region, coupling is very dependent on band-structure. Of note is the region where $E_{3}-E_{1}$ is close to $E_{g}$, which promotes resonant transitions; the transition probability between these excited electronic states increases dramatically, causing the Auger lifetime to drop, thus reducing $\tau_{c}$ by a factor of 2 .

In Figure $2 \mathrm{~b}$, we hold the bandgap approximately constant $\left(\mathrm{Al}_{0.35} \mathrm{Ga}_{0.65} \mathrm{As}_{y} \mathrm{Sb}_{1-y}\right.$ bariers), and adjust the arsenic concentration of the barriers, which has the largest on strain. We can adjust the strain in the barrier from positive to negative values by varying the amount of As. We see that influence of the band energies within the quantum well remains small, and this reflects little change in the Auger and spontaneous lifetimes. We conclude these structures are not dramatically strain driven.

We intend to grow several structures at University of Missouri (much like the previous investigation at $2 \mu \mathrm{m}$ ), to confirm these predictions in the confined excited state structures. This will also hopefully provide a suitable platform for demonstrating high power devices, at an unusual wavelength for type-I quantum well lasers. 


\subsubsection{Type-I Method: Strain Engineering}

Instead of being bound to preset strains based on the quantum well chosen and the lattice constant, relative to a binary compound (e.g. GaAs, GaSb, indium phosphide (InP), etc.), design-space would be dramatically opened up if the strain were considered another free parameter. While there are problems, which will be identified, it is possible to accomplish this through use of metamorphic buffer layers, which allow for nearly full relaxation of a ternary crystal on a binary substrate. This concept is based on Reference 27, in which a peak power of several hundred $\mathrm{mW}$ has been demonstrated, relying on metamorphic buffer layers to adjust the semiconductor crystal lattice constant to allow for different material composition and strain profiles to be grown on it.

NLCSTR performed a systematic optimization strategy and found regions which should improve the performance by more than about a factor of 20 when compared to Reference 27 and should allow for Watt-level room temperature cw performance. NLCSTR used its fully microscopic many-body models to calculate the intrinsic material properties of various quantum well structures for target wavelengths of about 2.9-3.2 $\mu \mathrm{m}$. All intrinsic properties relevant for laser performance such as gain and absorption as well as carrier losses due to radiaitive and Auger processes were calculated. The wells are assumed to be grown on metamorphic buffers that lead to an unstrained $\mathrm{Al}_{0.64} \mathrm{In}_{0.36} \mathrm{Sb}$ cladding.

Calculations were performed for target wavelengths of $2.9 \mu \mathrm{m}$ (that of Reference 27), and $3.2 \mu \mathrm{m}$, showing scaling to longer wavelengths. The well widths were varied between $6 \mathrm{~nm}$ and $13 \mathrm{~nm}$. Five different barrier compositions were assumed. Data were calculated for temperatures ranging from $250 \mathrm{~K}$ to $400 \mathrm{~K}$ and intrinsic carrier densities from the low absorptive to the high gain regime - in total 27 different structures were analyzed.

Type I quantum well structures for this wavelength regime are limited by strong carrier losses due to Auger processes. Thus, NLCSTR focused on looking for structural variations that lead to reduced Auger losses. Figure 13a shows the Auger loss time as function of the intrinsic carrier density at $300 \mathrm{~K}$ for the wells that were found to yield record performance in Reference 27 (dashed line), as well as for structures of various level of optimization. It was found that by varying the well width and barrier height, the Auger loss time can be reduced by almost three orders of magnitude for a given carrier density. This dramatic dependence is rather surprising and will be explored experimentally to confirm these results. Even if the reasoning is found to be sound, total non-radiative carrier lifetimes will be shorter, driven by terms such as SRH losses.

Figure 13b compares the threshold current density for the optimized structures from Figure 13a to that of the previously grown device[27]. The Auger losses are reduced with increasing optimization. However as discussed above, this also increases the threshold carrier density and changes the radiative loss current. Thus, despite a reduced Auger loss in "Optimization 1" as compared to the well in the previous study the threshold current is not reduced in that well.

This also leads to the fact that the threshold current reduction is not as strong as the reduction of the Auger losses. However, although the reduction of the threshold is about one order of magnitude smaller than the reduction of the Auger losses, the threshold reduction is still very significant - up to about a factor 25 for "Optimization 4." Further study seems to indicate the carrier loss is dominated by spontaneous emission; this situation is similar to the one typically observed in lasers for wavelengths of $1.3 \mu \mathrm{m}$ and shorter. This leads to high $T_{0}$ values and should allow for multi-watt level operation at room temperature.

University of New Mexico, Center for High Technology Materials (UNM) studied the growth of 

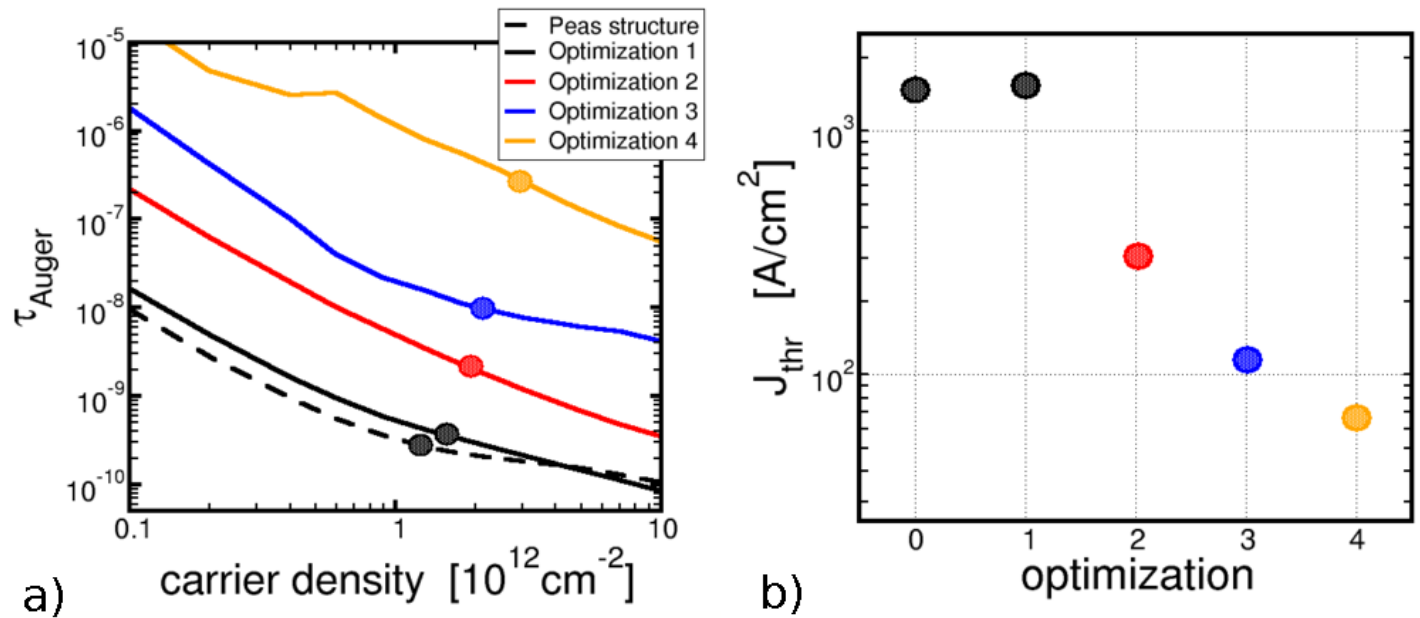

Figure 13. Equivalent thresholds and simulated Auger lifetimes for $\lambda \sim \mathbf{2 . 9} \mu \mathrm{m}$ devices a)Simulated Auger lifetimes as a function of sheet carrier density for structures grown latticematched to $A l_{0.64} I_{0.36} S b$. These include the previously grown structure (black dash) operating at $2.9 \mu \mathrm{m}$, and perturbations (colored) which amount to simply lower barrier heights. The dots mark the threshold carrier density for typical intrinsic optical losses. b) (Equivalent) threshold current densities for optimized structures.

metamorphic buffers on GaAs and GaSb substrates. It was found that substrates based on GaSb show less cross-hatching. This indicates a better growth quality with less defects due to strain mismatch between the top buffer layer and the substrate. UNM continued this study investigating improved growth conditions for growing on GaAs. Lower substrate temperature and higher Sb-flux were found to yield improved growth quality.

Growth results were also compared to results obtained by using an IMF layer to relax the strain between substrate and buffer instead of a direct buffer growth, a technique introducted in Section 3.6. Characterization of the IMF result as well as the direct buffer growth on GaAs and GaSb using AFM and Nomarski showed virtually no change in the surface quality between the three techniques. Both characterizations showed extremely rough surfaces (greater than $20 \mathrm{~nm}$ root-mean-squared (RMS) via AFM). The X-ray diffraction begins to differentiate the techniques, with the IMF interface technique clearly showing the GaSb peak next to the broad metamorphic peak and the straight metamorphic buffer approach showing a single broad metamorphic peak. The major consideration when moving an antimonide-based structure to GaAs is the optical properties. Using cryogenic PL to measure the intensity of a pair of InGaSb quantum wells grown on the different metamorphic buffers showed that the technique that yields the best PL is the straight buffer growth on GaSb substrate in Figure 14.

In general, the concept relies on the fundamental premise that lower barrier structures, which do little to confine the carriers, also have a exceedingly weak involvement from Auger recombination due to the difficulty in meeting the necessary momentum change in order to make the appropriate conduction band transition, introduced in Section 3.2. In such structures the carriers are not strongly confined in the wells and significant numbers of holes and/or electrons can spill from the well into the barrier, resulting in a leakage current, thus reducing the quantum well efficiency. 

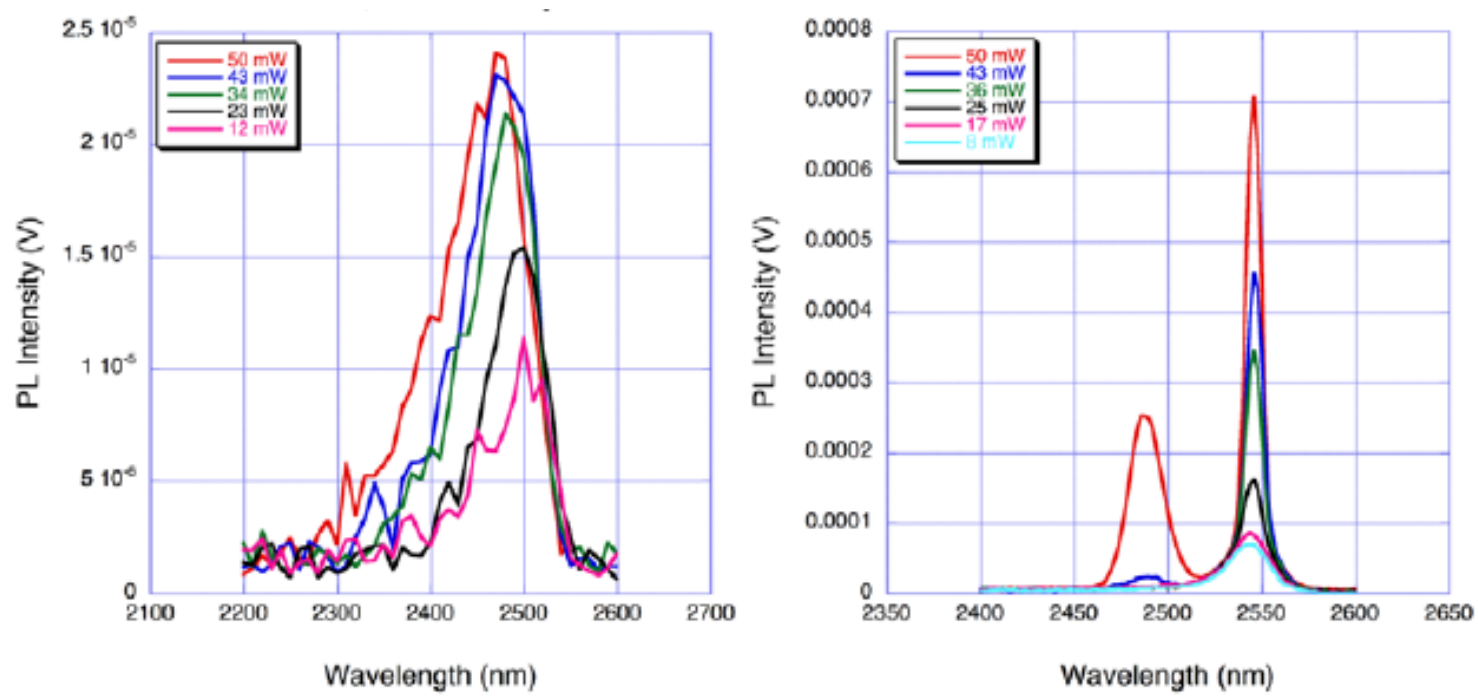

Figure 14. Measured PL from $77 \mathrm{~K}$ to $300 \mathrm{~K}$ for devices operating at approximately $3 \mu \mathbf{m}$.

a) $300 \mathrm{~K}$ PL at different pump powers, $b$ ) $77 \mathrm{~K}$ PL at different pump powers for $I n_{0.5} G a_{0.5} S b$ $Q W s$ on $A l_{0.64} I n_{0.36} S b / G a S b$ metamorphic buffer layer on $\mathrm{GaSb}$.
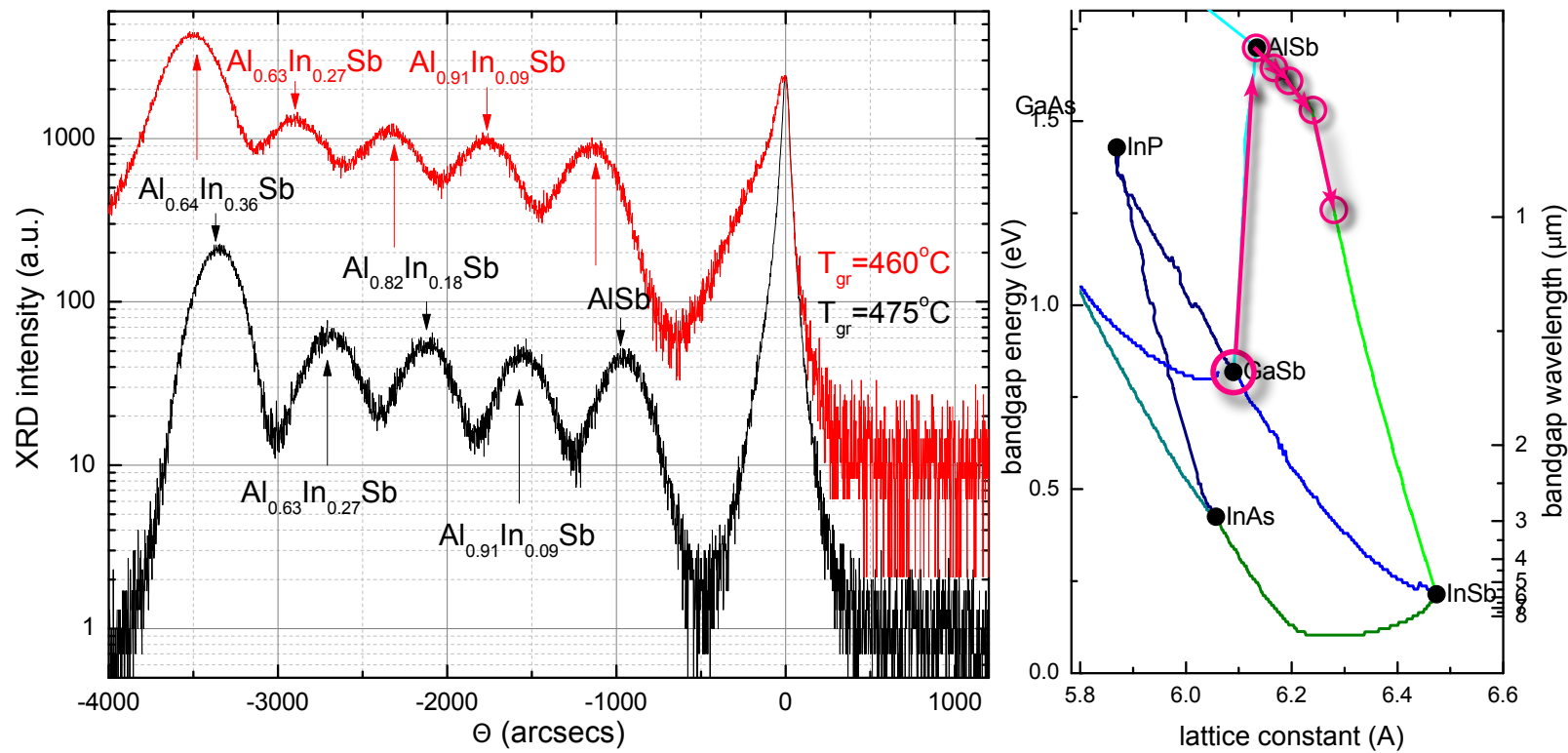

Figure 15. XRD for metamorphic buffer layer grown material designed to operate at approximately $3 \mu \mathrm{m}$.

From The structure is converted from GaSb to AlSb with layers slowly introducing increasing amounts of indium into the material. The lattice-constant is also given for each layer step. 

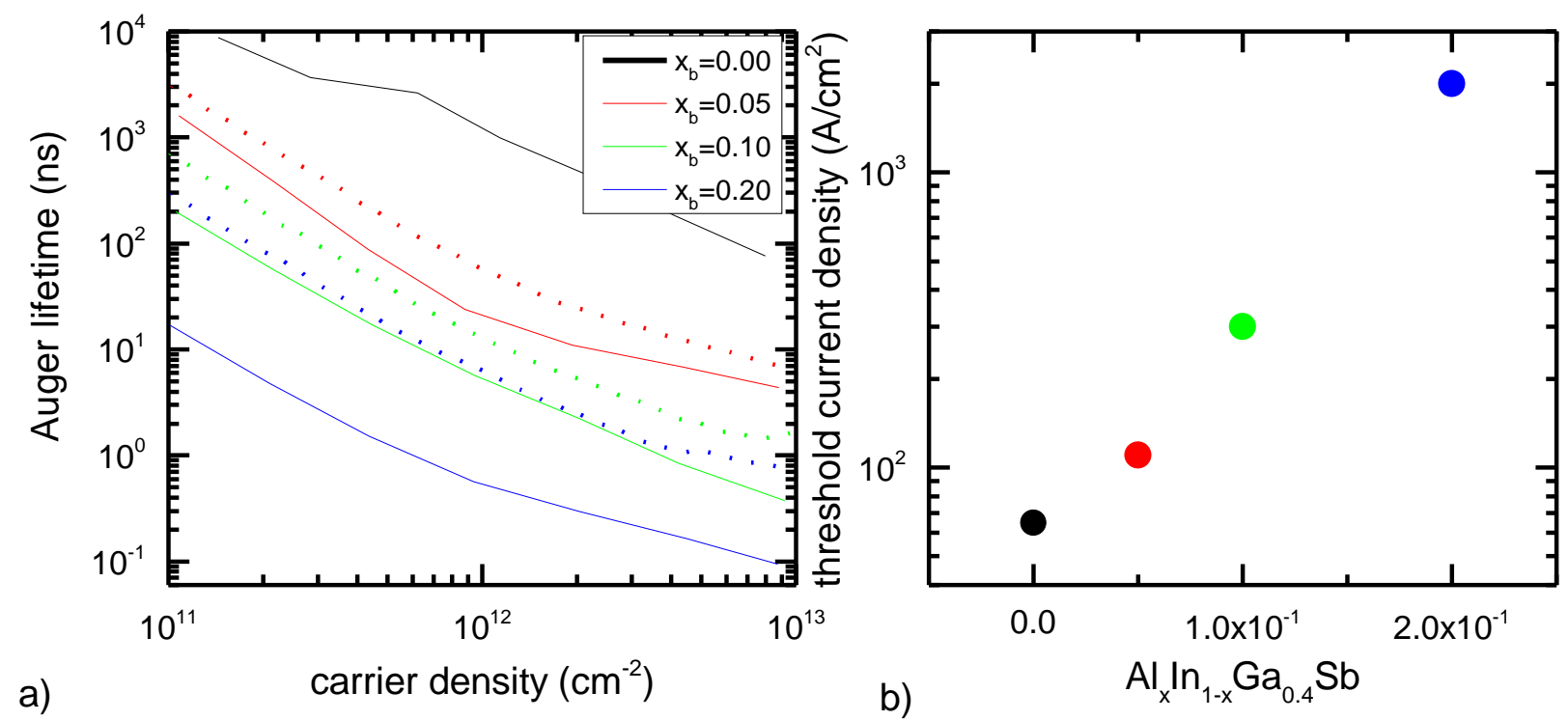

Figure 16. Metamorphic Buffer Layer grown material designed at approximately $3 \mu \mathrm{m}$. a) Auger lifetime calculated as a function of sheet carrier density which include a bulk treatment of the barrier material (solid lines) as well as a quantized subband model of the barriers (dashedlines). b) Calculate threshold current density as a function of barrier aluminum composition.

To simulate these structures, a thorough model similar to to that introduced in Section 3.1 is used to calculate gain and losses on this structure. Because the calculations are built up from basic principals, there is little need to alter the theoretical approach to calculating the gain on a non-binary substrate. Besides Auger losses, free carrier absorption (intraband absorption) losses are generally expected to limit the performance of devices in this wavelength regime. Additionally, for realistic applications, the far field broadening has to be limited, as previous designs would result in impractically high broadening[27].

Beginning from designs presented in Reference [27], devices were simulated adjusting the barrier material composition, which adjusts the $\Delta E_{c}$ and $\Delta E_{v}$. The initial design is similar to the "deep-well" structure presented in Section 3.1, although at a longer wavelength. Figure 16a shows the increase in Auger lifetime as a function of carrier density .

Typically, the use of a bulk model is used to simulate barrier states, thus easing the calculation requirements. However, it seemed that based on results in Figure 16a, there is a significant difference in the Auger-calculated when including subbands in barriers. Such a discrepancy between the results for both models had not been encountered in testing the codes for materials at shorter wavelengths - around 1 micron. In the latter case, the results of the subband model where found to depend somewhat on the barrier width and converged toward the results of the bulk model in the limit of wide barriers. The reason for the discrepancy between the results of the two models for the $3 . x$-micron materials is yet to be determined. Tests of the subband model for various barrier widths have shown an insignificant dependence and did not indicate a convergence toward the bulk model results. However, when comparing to literature[14], it seems that the barrier subband model more faithfully predicts the published results. One should note this is only as good as the reported growth structure matches what is grown. 


\section{Table 2. Wall plug efficiency characterization}

Tabulated laser performance for typical published results at around $2 \mu \mathrm{m}$ and $3 \mu \mathrm{m}$.

\begin{tabular}{c|c|cc}
\hline \hline & & $\sim 2 \mu \mathrm{m}$ & $\sim 3 \mu \mathrm{m}$ \\
$\eta_{D}$ & $\mathrm{~W} / \mathrm{A}$ & $0.3[28]$ & $0.15[14]$ \\
$V$ & $\mathrm{~V}$ & $1[28]$ & $1.5[14]$ \\
$\tau_{c}$ (pre-refined) & $\mathrm{ns}$ & 1 & .02 \\
$J_{t h}$ (pre-refined) & $\mathrm{A} / \mathrm{cm}^{2}$ & 200 & 3200 \\
$\tau_{c}$ (post-refined) & $\mathrm{ns}$ & 10 & 1 \\
$J_{t h}$ (post-refined) & $\mathrm{A} / \mathrm{cm}^{2}$ & 80 & 106 \\
\hline \hline
\end{tabular}

\subsubsection{Practical Implications}

Within this discussion, it is easy to lose sight of how this reduction in parasitic carrier losses affects the device. Figure 8 shows that reducing Auger currents directly affects the lasing threshold of the device. For one-dimensional devices, and excluding changes to thermal conditions, once the threshold gain has been achieved, the slope-efficiency is only dependent on the fraction of out-coupling loss to total loss. As such, wall plug efficiency (WPE) may be presented as:

$$
\eta_{W P E}\left(J>J_{t h}\right)=\frac{\eta_{D}\left(J-J_{t h}\right)}{J V},
$$

where $\eta_{D}$ is the differential efficiency of the laser, and $V$ is the device voltage. Typical values for these parameters are given in Table 2.

When analyzing improvements to a device's wall plug efficiency, we'd like to compare the efficiency of two devices at the same output power. Therefore, it helps if we restate Equation 6 as a function of output power:

$$
\eta_{W P E}(\text { Pout }>0)=\frac{P_{\text {out }}}{\left(P_{\text {out }} / \eta_{d}+J_{\text {th }}\right) V},
$$

We then plot a ratio of Equation 7, for two different values of $J_{t h}$ wherein the parasitic recombination rates change the threshold current. This improvement can be dramatic for smaller powers, and asymptotically reaches unity for large powers. The lasers defined in Table 2 are plotted in Figure 17. 


\subsection{TYPE-II QUANTUM WELL SURFACE-EMITTING DISTRIBUTED FEEDBACK LASER}

To counter the Auger problem introduced in Section 3.0 and extend to longer wavelengths, it becomes advantageous to look to a non-homogeneous quantum-well structure. The type-II "interband" cascade laser achieves this by confining the holes in a center "well" while confining the electrons in layers immediately adjacent. If the layers are sufficiently thin, the wavefunction overlap can be considerable, as much as $\sim 70 \%$ that of a type-I quantum-well.

Carriers may be optically[29, 30] or electrically[31] excited. Optically pumped lasers have demonstrated outstanding results and are predicted to be able to scale from 2.5-9 $\mu \mathrm{m}[32]$ at cryogenic temperatures. At low temperatures, these devices exhibit multi-Watt performance, but this drops off dramatically at higher temperatures[30]. The electrically injected lasers are most often employed in a cascade structure (quantum wells excited serially), and this realization has been termed the interband cascade laser (ICL). ICLs have been employed at temperatures up to $340^{\circ} \mathrm{C}[31]$ and have demonstrated over $0.5 \mathrm{~W}$, cw operation.

In this AFRL/RY type-II quantum-well laser project, the goal was to investigate the employment of type-II quantum wells with a surface-emitting distributed feedback (SE-DFB) cavity embodiment. Two of the most important steps to develop the SE-DFB laser are to:

1. develop an appropriate exposure technique and process for the grating features

2. develop an effective technique to transfer this pattern from photoresist into semiconductor

\subsection{Type-II Method: Active Region Modeling}

In discussions with NLCSTR, we are pleased to discover that Simulase ${ }^{\circledR}$, the software we are using for rigorous many-body gain and carrier loss calculations in type-I structures (outlined in Section 3.0) is also

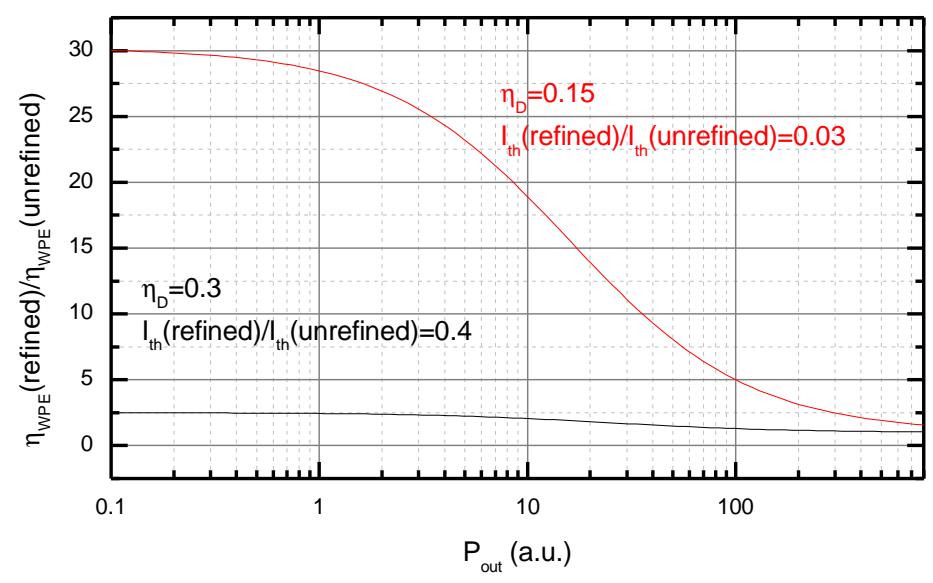

Figure 17. Improvements to wall-plug efficiency.

Analytical wall-plug efficiency improvements based on reasonable values for reduction in parasitic currents such as Auger and spontaneous recombination. The black curve represents

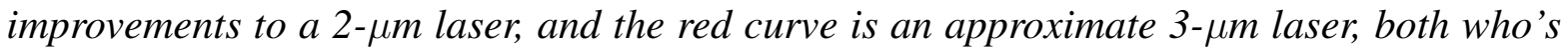
values are given in Table 2. 


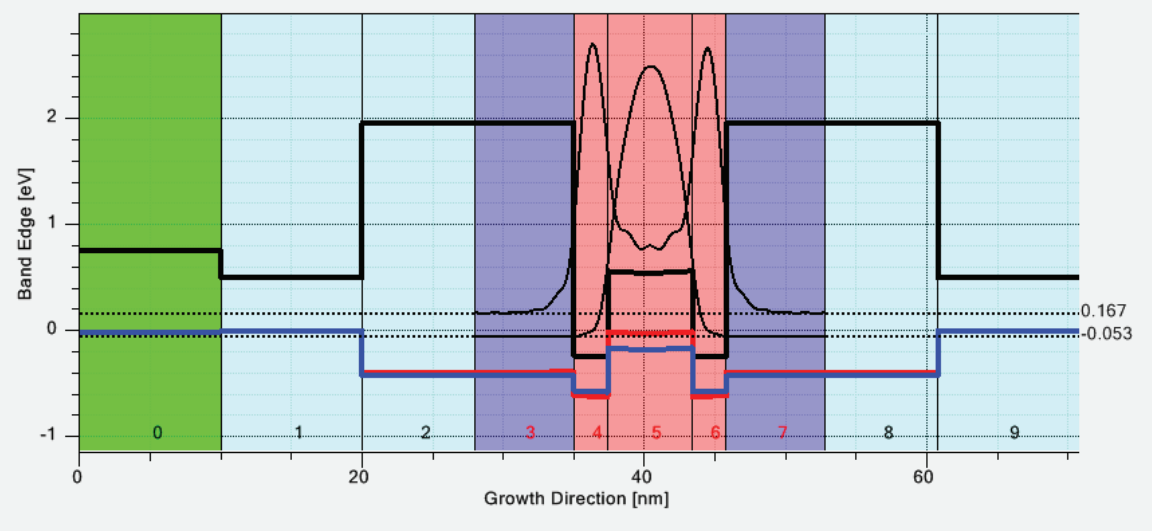

Figure 18. Standard type-II quantum well laser band schematic.

The three-layer structure creating the quantum well is composed of a hole confinement layer flanked by electron-confinement layers. When these are sufficiently small, the wavefunctions overlap significantly, aiding in device efficiency.

able to adequately predict gain in type-II quantum wells. The first step is to compare published active regions in the literature to those predicted by the software. This effort is somewhat challenged because the reports of ICLs are rarely thorough and often do not provide sufficient detail of the active region to simulate faithfully.

The primary concern in type-II quantum-well lasers is wavefunction overlap. The quantum-well is a three-layer structure, flanked by barriers. Hole confinement (valence-band vacancies) is typically accomplished in a small central layer, and is most often flanked by two narrow electron confinement layers (electrons confined in the conduction band). Figure 18 shows a typical structure designed for electrical injection after Reference 11. The design often relies on strong confinement of the carriers within the three wells through use of wide-bandgap barriers made of aluminum arsenide (AlAs). While this is a very effective approach for electrically excited devices, optically excited devices require narrower gaps in which the pump light undergoes single-pass absorption. Unfortunately, as one reduces the bandgap of the barriers to absorb pump photons, both the electron- and hole-confinement becomes more bulk-like and the overlap (as well as probability of locating the carrier within the well) drops dramatically.

\subsubsection{Double-Confined Structure}

Potentially, to utilize narrow-bandgap barrier material, it becomes advantageous to implement a narrow-band absorbing layer within a secondary confinement region (notated as "secondary confinement" in Figure 19) which confines both the holes, and to a lesser extent the electrons upon weak confinement of the narrow-gap "barriers" of the absorption layers. Figure 20a-d show the wavefunctions of the lowest excited wavefunctions of the conduction and valence bands as a function of the secondary confinement layer material. The spacer layer was the traditional AlAs material. In the case of small indium compositions (Figure 20a,b), the secondary confinement structure is very influential in defining the wavefunction, where the center hole well is readily coupled to the secondary confinement. For large indium concentrations, the smaller bandgap (Figure 20c,d) promotes hole wavefunctions which are mostly confined within the center hole well. although 


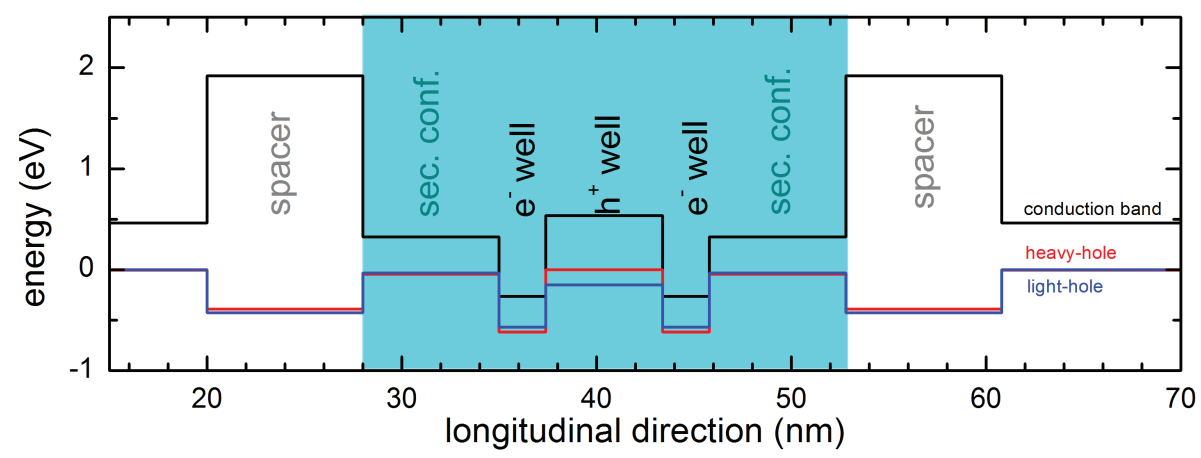

Figure 19. Conceptual approach to the double-confined structure

the electron wavefunction remains strongly influenced by the secondary confinement structure. Not surprisingly, as the wavefunctions become more localized (e.g. Figure 20d) the gain increases. This is depicted in Figure 20e, which shows the calculated absorption of such structures given in Figure 20a-d.

It should be noted these structures are very influenced by the width of the secondary confinement structure. In order to achieve sufficient pump absorption, the secondary confinement layer must be too wide (Beer-Lambert law says absorption $\propto 1-e^{-\alpha d}$ where $\alpha$ is the material absorption and $d$ is the material thickness). The designs in Figure 20 have pump absorptions on the order of $1 \%$ because the total thickness is too small, even for 10 repeats of the structure. We must, therefore utilize a more traditional structure utilizing extremely thick absorber layers without the double-confined structure.

\subsubsection{Single-Confined Structure}

The weakly confined structures created with the narrow-bandgap barrier materials limit the short-wavelength limit because at some point, the electrons cease to remain confined. Figure 21 shows result of parametrically adjusting the hole well thickness (reported in monolayers) and its influence on absorption/gain.

A few notes as a result of this. The thicker hole-well decreases the $\Delta \mathrm{E}_{g}$, therefore this results in a longer wavelength. The model has a systematic error (a structure we know should emit at $4.3 \mu \mathrm{m}$ is predicted numerically to emit at $6.98 \mu \mathrm{m}$ ). Although the actual source of this error is still being investigated, we suspect the exact subband positions are not being calculated accurately. While these provide a less relevant effect for type-I lasers, type-II lasers are particularly sensitive to these positions. At thinner hole well thicknesses, the holes are no longer confined as they couple to the $\mathrm{In}_{0.4} \mathrm{Ga}_{0.6} \mathrm{AsSb}$ material.

\subsection{Device Design}

We have focused on two structures, one with five wells, and one with seven. These should provide sufficient pump absorption and gain. The designs result in decent modal gain overlap $(\sim 4 \%)$, and single transverse mode operation. The most difficult design parameter is the modal grating overlap which will provide the "mirror" in the distributed feedback laser. This overlap would ideally be about $1.5 \times 10^{-4}$, although our present designs are limited to about a $\Gamma_{\text {grat }}$ of $5 \times 10^{-5}$. These structures have been sent to be grown 


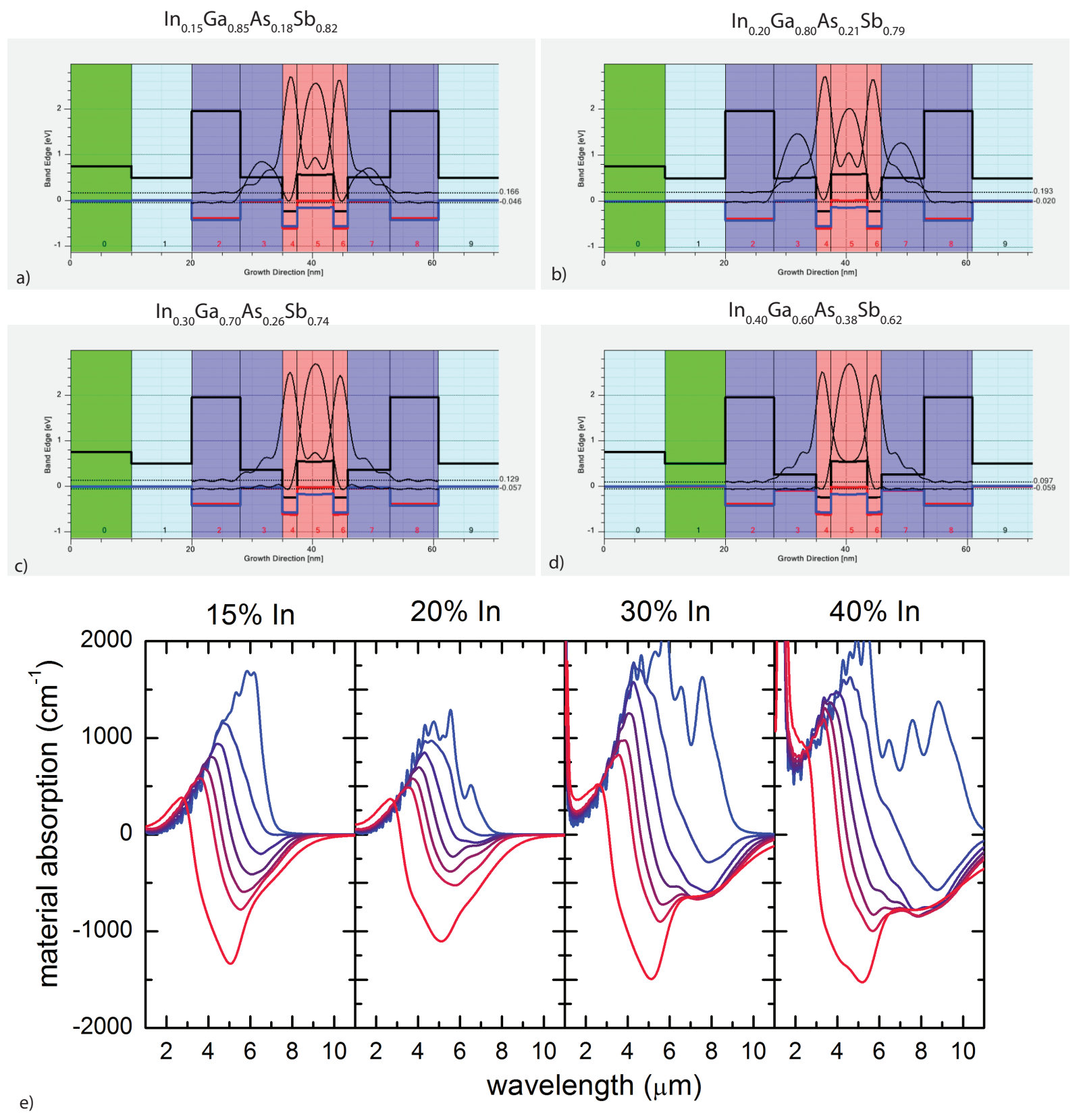

Figure 20. Absorption and wavefunction calculations for type-II quantum well active region

Calculations showing wavefunction calculations along with absorption (negative gain) calculations utilizing many-body theory.The curves in (e) represent a different carrier density: 0.1 (blue), $1,2,3,4,5$, and $10($ red $) \times 10^{12} \mathrm{~cm}^{-2}$. 


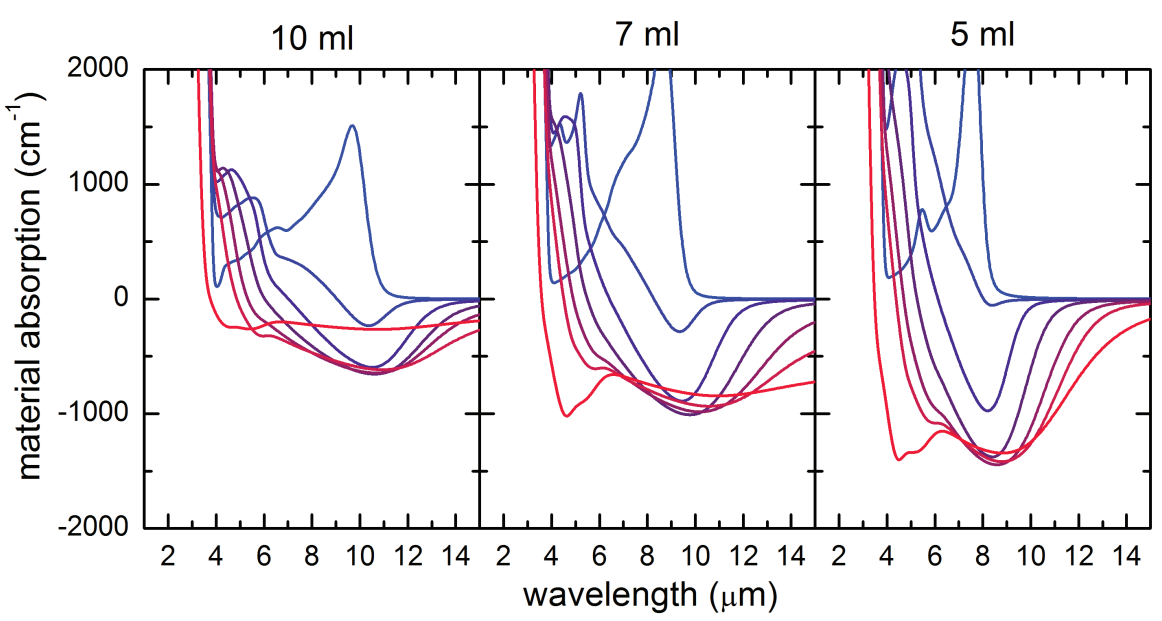

Figure 21. Influence of hole-well thickness

Calculations showing absorption and influence of the hole-well thickness. The curves represent a different carrier density: 0.1(blue), 1, 2, 3, 4, 5, and $10($ red $) \times 10^{12} \mathrm{~cm}^{-2}$.

commercially and returned to fabricate, mount, and test.

\subsection{Type-II Device Modeling Results and Discussion}

\subsubsection{Introduction}

When designing SE-DFBs, the most critical influence over the device are the grating-waveguide parameters $\zeta, \kappa$, and $\alpha_{\text {surf }}$ in the coupled-wave equations. Their derivation begins with approximations for estimating these parameters. Rough rules of thumb for approximating their values are derived. This is followed by an outline of a potential technique for computing the parameters for general grating-waveguides (not just rectangular gratings).

\subsubsection{Coupled Differential Equations}

The differential equations suitable for coupled distributed feedback (DFB)s which include surface emission are given by the seminal paper of Noll, and Macomber[33]. First, the total field is assumed to be of the form:

$$
E(x, z)=R(z)\left[\frac{E^{+}(x, z)+E^{-}(x, z)}{2}\right]+S(z)\left[\frac{E^{+}(x, z)-E^{-}(x, z)}{2}\right]
$$

where $E^{+/-}$are considered the right- and left-going waves, and $R$ and $S$ are defined as normalized amplitude functions. With these considered, along with the coupled wave equations, the coupled partial differential equation (PDE) set for $R$ and $S$ are solved by the same manuscript to be:

$$
\begin{aligned}
\frac{\partial R(z)}{\partial z}-[\alpha(z)+i \delta+i \zeta] R(z) & =i \kappa S(z) \\
-\frac{\partial S(z)}{\partial z}-[\alpha(z)+i \delta+i \zeta] S(z) & =i \kappa R(z)
\end{aligned}
$$


where $\delta$ defines the finite length of the resonant wave vector, $\alpha$ is the waveguide propagation loss (mentioned below), and $\zeta$ is defined relative to the infinite length problem.

\subsubsection{Approximate Estimates of Grating Parameters}

A general approach to finding the grating-waveguide parameters in a manner consistent with rigorous boundary conditions was described by Noll for transverse electric (TE) modes of rectangular gratings[33]. A later paper by Finger et al. adopted the Noll approach for transverse magnetic (TM) modes of rectangular grating waveguides[34] - a formalism that would be necessary if deploying the SE-DFB for QCLs. Both papers solve for the two infinite-length solutions (i.e.have exact periodicity along the $z$-axis). The infinite-length gain-frequency eigenvalues are then used to find the grating parameters for the coupled-wave equations that describe finite-length devices.

Although no code is available for calculating the grating parameters, we are able to extrapolate from calculations that were tabulated in Noll. What we do have available are planar-waveguide codes. A planar waveguide code represents the grating layer with a duty-cycle weighted average of the dielectric constant values (i.e. $n^{2}$ ) on each side of the grating layer. What planar-waveguide codes can find is the optical confinement in the metal as well as a value of the metal loss.

An approximate estimate of the surface loss coefficient $\alpha_{\text {surf }}$ is given by

$$
\alpha_{\text {surf }} \approx 8 \Gamma_{g} / \Lambda
$$

where $\Gamma_{g}$ is the optical confinement fraction of the waveguide mode in the grating layer and $\Lambda$ is the grating periodicity. This formula is a rough fit (within a factor of two) to past modeling analysis results of 830-nm devices. Extrapolation to longer wavelength is included in the grating periodicity denominator ineq:alpahsurf as per the wavelength-coordinate scale invariance of the wave equation. The effect of varied metal index is less clear.

To gain insight into the coupled-wave equation parameters $\kappa$ and $\zeta$ it is useful to review their derivation. They are found from the mode gain-frequency eigenvalues $\alpha+i \delta$ of the two solutions $E^{+}$and $E^{-}$from idealized infinite-length problem (equivalent to exact periodic conditions). These values are related to the grating parameters as follows

$$
\kappa=\frac{1}{2}\left(i \alpha^{-}-i \alpha^{-}+\delta^{-}-\delta^{+}\right)
$$

and

$$
\zeta=\frac{1}{2}\left(i \alpha^{-}+i \alpha^{-}-\delta^{-}-\delta^{+}\right)
$$

The imaginary part of the eigenvalues can be partitioned into metal loss and surface loss parts

$$
\alpha^{+}=\alpha_{\text {metal }}^{+}+\alpha_{\text {surf }}
$$

whereas the $E^{-}$solution has no surface loss

$$
\alpha^{-}=\alpha_{m e t a l}^{-}
$$

The two metal loss parameters cannot be calculated without a complete code but their magnitude in Noll is typically on the order of the mode loss from planar models.

$$
\alpha_{\text {planar }} \approx \frac{1}{2}\left(\alpha_{\text {metal }}^{+}+\alpha_{\text {metal }}^{-}\right)
$$


The $\alpha^{+}$metal loss was always found to be greater than $\alpha^{-}$, although the two values tend to be closer together for weak gratings, which will be the case for the type-II SE-DFB designs created for this effort.

The real part of $\kappa$ and $\zeta$ are derived from the $\delta$ part of the eigenvalues and cannot be estimated from a planar model. However, it turns out that when the grating duty cycle is around 50 percent, which coincidentally gives the strongest surface output, $\delta^{-} \sim \delta^{+}$and Equations 12 and 13 can be adjusted accordingly.

Putting all these approximations together we arrive at the rule of thumb for $\kappa$ :

$$
\kappa \approx \frac{i}{2} \alpha_{\text {surf }}
$$

The phase of $\kappa$ is dependent on the coordinate system. It rotates over $2 \pi$ radians when the $z$-coordinate origin shifts over $\Delta z=\Lambda / 2$. The exact coordinate origin is not important in most analysis problems, therefore neither is the absolute phase of $\kappa$. The insignificance of the phase of $\kappa$ is verified in device models. It only becomes important in cases where devices with facet reflectors are modeled.

The value of $\operatorname{Im}(\zeta)$ is estimated from loss parameters whereas $\operatorname{Re}(\zeta)$ is unknown without the $\delta^{ \pm}$ eigenvalues. However, $\operatorname{Re}(\zeta)$ can be ignored because it simply redefines the zero of the $\delta$ axis. Hence, we arrive at the approximation for $\zeta$ :

$$
\zeta \approx \frac{i}{2} \alpha_{\text {surf }}+i \alpha_{\text {planar }}
$$

Equations 11, eq:kappa and eq:zeta provide a basis to proceed with device calculations. However, there is still uncertainty in the $\alpha_{\text {surf }}$ estimator in Equation 11 because it is based on a different metal-index value than occurs at $4 \mu \mathrm{m}$ wavelength.

\subsubsection{General Computation Method}

The single-layer rectangular gratings models in Noll and Finger led to difficult analytical and numerical problems. The root of the difficulty arises from the classical theory of deep gratings where the Rayleigh method breaks down. The field in the so-called "selvedge" region must have its own form and must be boundary matched to the adjacent layers. For example, a separate grating-layer eigenfunction method was employed in Noll. But just finding the eigenvalues within the grating-layer was difficult. The additional problems of matching boundary values at the edges of the grating layer and finding the laser-resonance eigenvalues made automated computation problematical.

Instead, we would like to have a method that efficiently and reliably models any grating-waveguide structure not just single-layer rectangular gratings. It would then be possible to analyze structures with mixed layers of dielectric and metal that are achievable with modern micro-fab technology. With such an analytical tool it may be possible to design practical grating-waveguides that achieve both strong coupling and low metal loss.

One possibility examined in the past is to put the problem on a grid. Steady-state solutions can be found by iteration or simulation of time evolution. While such techniques can work, they have a problem in that the frequency eigenvalue should be accurate to $\delta \sim 0.01 \mathrm{~cm}^{-1}$ out of $n k \sim 1 \times 10^{4} \mathrm{~cm}^{-1}$ or one part in $10^{6}$. But errors from finite grid size and iteration noise can be on this order of magnitude. Moreover, grid techniques are computationally intensive (i.e.very slow to run). 
Floquet-expansion methods such as used in Noll and Finger can find the resonance eigenvalues to high precision. The issue is instead how to accurately and reliably solve the equations for each order function with arbitrary grating shapes. The order functions are defined by the set of coupled differential equations

$$
\frac{d^{2}}{d x^{2}} E_{m}(x)-K_{M}^{2} E_{m}(x)+k^{2} n^{2}(z) E_{m}(x)=k^{2} \sum_{q=-\infty, \neq 0}^{\infty} \epsilon_{q}(z) E_{m-q}(x)
$$

The grating structure is contained in the dielectric function $\epsilon(x, z)$ the Fourier transform of which $\epsilon_{q}(x)$ which appears in the previous equation. More formally, the problem is to solve the coupled set of differential Equation 19 subject to the boundary conditions that there are outgoing waves only - whether radiating or evanescent. All of the non-grating layers have the general form of solution

$$
E_{m}(x)=A e^{i p x}+B e^{-i p x}
$$

so that the outgoing-wave boundary conditions can be expressed as

$$
E_{m}(x)=\left\{\begin{array}{cl}
A e^{i p_{m} x} & , \quad x>x_{\max } \\
B e^{-i p_{m} x} & , \quad x<x_{\min }
\end{array}\right.
$$

where $x_{\min }$ and $x_{\max }$ are the interfaces to the outer-most layers and where

$$
p_{m}=+\sqrt{n^{2} k^{2}-K_{m}^{2}} .
$$

The eigenvalue problem of second-order, ordinary differential equation (ODE) with boundary conditions on both sides of the field generally have well-defined solutions.

\subsubsection{Shooting-Method Numerical Technique}

In the fully self-consistent solution, each equation for $E_{m}(x)$ in Equation 19 constitutes a non-homogenous ODE. The zero-order equation $(m=0,-2)$ is just the standard planar- waveguide equation when the other $E_{m}(z)=0$. Only specific values of $\beta=\widetilde{n} k$ allow the zero-order equation to satisfy the outward-going boundary conditions on both sides. These are the usual waveguide modes.

Planar modes are found by testing trial $\beta$-values (also be expressed as $\delta \equiv \delta \beta$ value) to see if they satisfy the boundary conditions. The classical method is to evaluate the determinant of the boundary-matching equation matrix. This works for simple layers of uniform index. Alternately, a "shooting" method can integrate the differential equation to the opposite side of the structure given two starting points at one edge. These two points can easily be generated because the form of solution is analytic, the initial amplitude is arbitrary, and the $\mathrm{p}$-value is known for any given trial- $\beta$ value. In general, the solution of the far side has the form given in Equation 21 and - depending on which side you start - the value of the $A$ or $B$ coefficient should vanish when a mode is found. Thus one can construct a test function such as

$$
\mathcal{T}=\frac{|B|^{2}}{|A|^{2}+\gamma}
$$

which blows up when $A \rightarrow 0$. The steepness of the test function makes it easy to zero in on precise eigenvalues. The small parameter $\gamma$ avoids division-by-zero errors. The normalization of $E_{0}(x)$ is arbitrary but it is conveniently chosen to be power integrated to unity. 
The shooting method for finding $E_{0}(z)$ can still be applied when the $E_{m}(z)$ functions are non-zero. The gain-frequency values will just shift a little. In fact, the gain-frequency eigenvalues of the zero-order equation when self-consistent order functions are found are the very values of $\alpha_{i} \delta$ that we seek for computing $\kappa$ and $\zeta$.

The above discussion outlines how the zero-order problem is handled. That still leaves the other orders $m \neq 0,-2$. But the problem is still the same-finding solutions to the non-homogeneous ODE subject to out-going-wave boundary conditions. The difference is that the amplitude of the fields cannot be subjected to normalization but must be consistent with the non-homogenous term in the ODE. A shooting method can be used again except in this case there is no eigenvalue search. Instead a search can to be done on the real and imaginary part of the $A$ or $B$ coefficient launched on one side of the structure such that the outgoing wave boundary condition is satisfied on the opposite side of the structure. This is done for each order and the whole process is iterated until it converges. We can anticipate that an iterative method will converge quickly because the grating layer is only a perturbation on the main mode.

The above outlines the method. There are many other details such as use of analytic solutions whenever possible to speed up the integration and the method of numerically integrating across the metal-semiconductor interface. The TM problem gets a little more complicated but can still be expressed as an ODE (with a new derivative term) and also solved using a shooting method. While we choose to apply this type of cavity to the type-II quantum well, where TE calculations are the most appropriate, quantum cascade lasers are entirely TM polarized, and therefore the coupled mode solution would be required for creating a QCL SE-DFB.

\subsubsection{Optical Gain Parameters}

The material gain parameters including the internal-quantum efficiency $\left(\eta_{i}\right)$, the current- gain coefficient $A_{j}$, and the gain offset $b$ are used in the unsaturated gain formula:

$$
g=\Gamma\left(\frac{\eta_{i} A_{j} J}{d}-b\right)
$$

where $J$ is the equivalent current density, and $d$ is the quantum-well thickness. These and other parameters can be inferred from a publication on optical pumping of type-II semiconductor lasers[35], which described an epitaxial structure similar to that in our project. These devices had optical-to-optical efficiency of $18 \%$ at $83 \mathrm{~K}$, which corresponds to differential quantum efficiency (DQE) of 0.37 after correcting for the quantum defect from $1.85 \mu \mathrm{m}$ to $3.8 \mu \mathrm{m}$. Other parameters given in the paper include: the front- and back-facet reflectivity $=0.02$ and $=0.3$, respectively; the mode loss $\alpha=3 \mathrm{~cm}^{-1}$ and the optical pumping "stripe" area $W \times L \approx 250 \times 2500 \mu \mathrm{m}$. The authors do not estimate the internal quantum efficiency $\eta_{i}$ but one can back out a value from the DQE formula for front-facet emission

$$
D Q E=\eta_{i} \frac{\frac{1}{2 L} \ln \left(\frac{1}{R_{f}}\right)}{\frac{1}{2 L} \ln \left(\frac{1}{R_{f} R_{b}}\right)+\alpha}
$$

Equation 25 and the data in Reference 35 imply $\eta_{i}=0.63$. The threshold mode-gain coefficient can be calculated from the standard threshold gain condition (gain equaling losses):

$$
g_{t h}=\frac{1}{2 L} \ln \left(\frac{1}{R_{f} R_{b}}\right)+\alpha
$$


Table 3. Calculated and assumed waveguide parameters for two structures sent to be grown.

\begin{tabular}{|r||r|r|}
\hline & KM8_060_WG3_7x & KM8_060_WG3_5x \\
\hline \hline$N_{w}$ & 7 & 5 \\
\hline$n_{\text {eff }}$ & 3.45205 & 3.28149 \\
\hline$\alpha_{\text {metal }}$ & $3.0333 \mathrm{~cm}^{-1}$ & $6.1513 \mathrm{~cm}^{-1}$ \\
\hline$\Gamma$ & .005007 & .03233 \\
\hline$\Gamma_{\text {grat }}$ & $4.81 \times 10^{-5}$ & $1.01 \times 10^{-4}$ \\
\hline
\end{tabular}

which predicts $g_{t h}=13.2 \mathrm{~cm}^{-1}$. The threshold pump power reported is $P_{t h} \approx 2 \mathrm{~W}$, and may be converted to an equivalent threshold current density using the following equation:

$$
J_{\text {equiv }}=\frac{e \lambda_{p}}{h c} \times \frac{P_{t h}}{W L}=480 \mathrm{~A} / \mathrm{cm}^{2}
$$

where $\lambda_{p}$ is $1.85 \mu \mathrm{m}$ used in Reference 35. The optical confinement and active thickness were not given in the paper. However, we have analysis of similar epitaxial structures in the early part of this project with seven active zones[25]. That design had and $\Gamma=9 \times 10^{-3}$ and $d=46.2 \mathrm{~nm}$, referenced to $66 \mathrm{~nm}$ combined thickness of the electron and hole confining layers in each quantum region. The paper had ten quantum regions but the ratio should be about the same, and therefore we will assume $\Gamma=0.0129$ and $d=66 \mathrm{~nm}$ for the reported structure. The $b$ parameter is unknown as we have only one data point to fit a straight line, but we do know that $b>0$. We therefore guess that $b \approx 300 \mathrm{~cm}^{-1}$ (comparable to practical values for GaAs). Then from Equation 24 we estimate $A_{j}$ as:

$$
A_{j}=\frac{d}{\eta_{i} \Gamma J_{t h}}\left(g_{t h}+\Gamma b\right) .
$$

\subsubsection{Grating Parameters}

There are two epitaxial designs to be considered in the device modeling runs. They will be assumed to have the same $A_{j}$ and $b$, gain and free-carrier mode-loss parameters as estimated above. The waveguide parameters for the two epitaxial designs are known. Other parameters will be assumed to have properties that were estimated in Reference 36. The prediction in the device modeling runs that follows are an attempt to be more predictive of actual performance. Particular waveguide parameters are given in Table 3.

\subsubsection{Device Designs}

With estimates of the basic material parameters established, we can now turn to the simulation of specific device designs. Three stripe sizes were considered: $300 \times 2000,200 \times 3000$ and $150 \times 4000 \mu \mathrm{m}^{2}$ (the stripe area is designed to be the same in each case). For each stripe design, both the 7- and 5-quantum region materials were modeled. Other design details include the unpumped grating dimensions, the grating curvature, and the grating chirp. Particular parameters of grating curvature will depend on real waveguide designs as well as thermal profiles. 
Table 4. Numerical results of laser modeling

\begin{tabular}{|c||c|c|c|c|c|c|c|c|c|}
\hline $\begin{array}{c}\text { stripe size } \\
\left(\mu \mathrm{m}^{2}\right)\end{array}$ & $\begin{array}{c}\text { epi } \\
\text { epi }\end{array}$ & $M$ & $\begin{array}{c}g_{\text {th }} \\
\left(\mathrm{cm}^{-1}\right)\end{array}$ & DQE & $\begin{array}{c}J_{\text {th }} \\
\left(\mathrm{A} / \mathrm{cm}^{2}\right)\end{array}$ & O-O eff & $\begin{array}{c}\theta_{\text {lat }} \\
(\mathrm{deg})\end{array}$ & $\begin{array}{c}\theta_{\text {long }} \\
(\mathrm{mrad})\end{array}$ & Strehl \\
\hline \hline $300 \times 2000$ & 5 & 1.77 & 19.4 & 0.20 & 620 & 0.088 & 15.6 & 1.56 & 0.11 \\
\hline $300 \times 2000$ & 7 & 1.77 & 12.8 & 0.21 & 666 & 0.088 & 15.8 & 2.50 & 0.05 \\
\hline $200 \times 3000$ & 5 & 1.91 & 18.3 & 0.21 & 556 & 0.093 & 8.0 & 0.61 & 0.61 \\
\hline $200 \times 3000$ & 7 & 1.91 & 11.3 & 0.22 & 591 & 0.098 & 7.7 & 0.55 & 0.51 \\
\hline $150 \times 4000$ & 5 & 2.04 & 17.1 & 0.20 & 521 & 0.088 & 5.2 & 0.48 & 0.85 \\
\hline $150 \times 4000$ & 7 & 2.04 & 10.4 & 0.22 & 519 & 0.093 & 5.3 & 0.43 & 0.81 \\
\hline
\end{tabular}

The total grating length was chosen to be long enough to capture most of the emitted radiation. However, the total grating length and magnification are constrained in that the in-plane virtual sources should be located outside the grating. This can occur when the unpumped part of the grating is very long or the magnification is very high.

The grating curvature is adjusted to attain a nominal stripe magnification of $M \approx 2$. This rough rule of thumb gives strong enough curvature to suppress filamentation (the amplitude-phase coupling prevalent in many semiconductor lasers), yet is small enough to make the beam quality easy to achieve (ultra-high curvature designs usually have poor beam quality). The grating chirp was used to optimize the beam quality in each case.

The grating was made wide enough to avoid reflections from the edges. It was found that an insufficiently wide grating led to filamentation. A similar effect was found in a study of electrically-pumped SE-DFB devices. The softly extended edge of the Gaussian pump beam as compared to a sharply-defined electrical contact stripe makes this issue even more important for optically-pumped devices.

It is important to note that facet reflection can destabilize devices both spatially and spectrally. Past GaAs-based devices used absorbers zones to suppress facet reflections. An alternative method may be where the laser longitudinal mode is intentionally misaligned with respect to the crystal cleave direction (tilted) so that end-reflections are deflected off-axis.

\subsubsection{Modeling Results and Discussion}

Table 4 summarizes the results from the numerical modeling of the six designs. The gratings in all designs had to be extended $2.5 \mathrm{~mm}$ beyond the end of the stripe in order to reduce end losses. In comparison, they only need to extend $0.4 \mathrm{~mm}$ for typical $1-\mu \mathrm{mGaAs}$-based SE-DFB devices, essentially scaling with wavelength. The longer end-length results from the smaller surface-diffraction coefficient.

The $300 \times 2000 \mu \mathrm{m}$ "stripe" designs lased, but had much lower beam quality and more instability than the other stripe designs. The grating in that case had to be very wide to avoid outer-edge reflections, otherwise filamentation would set in. The narrower designs were better behaved and had good beam quality, especially the $4 \mathrm{~mm}$ long designs, which achieved a Strehl of 0.8 , performing very nicely for lasers of this type.

The field and beam characteristics from one of the $4 \mathrm{~mm}$ stripe designs are shown in Figures 22 and 23. 
stripe \& grating

Gain field

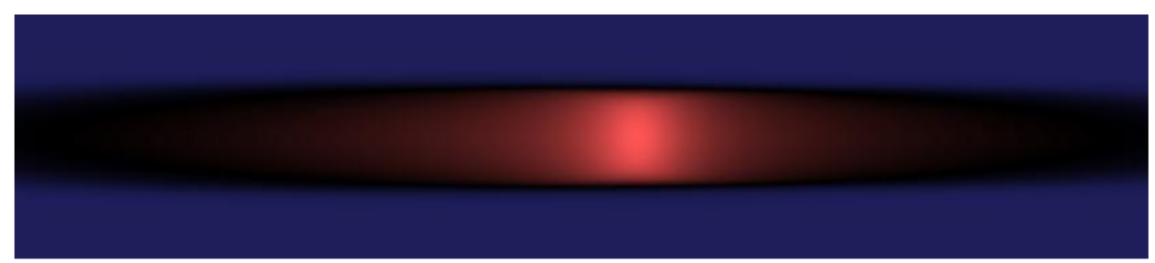

$R(z)$

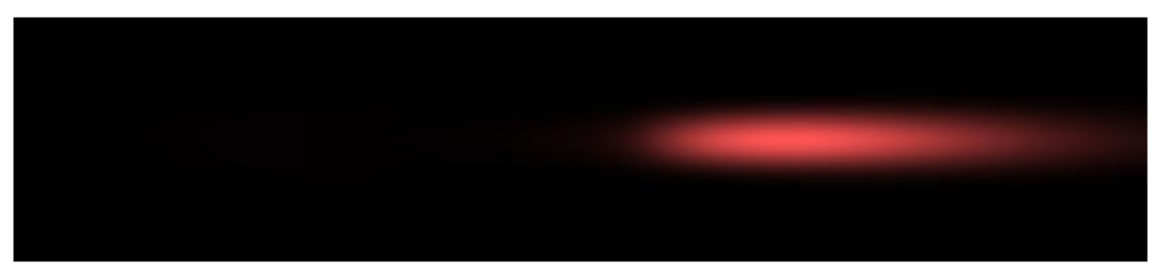

$S(z)$

Near field at grating
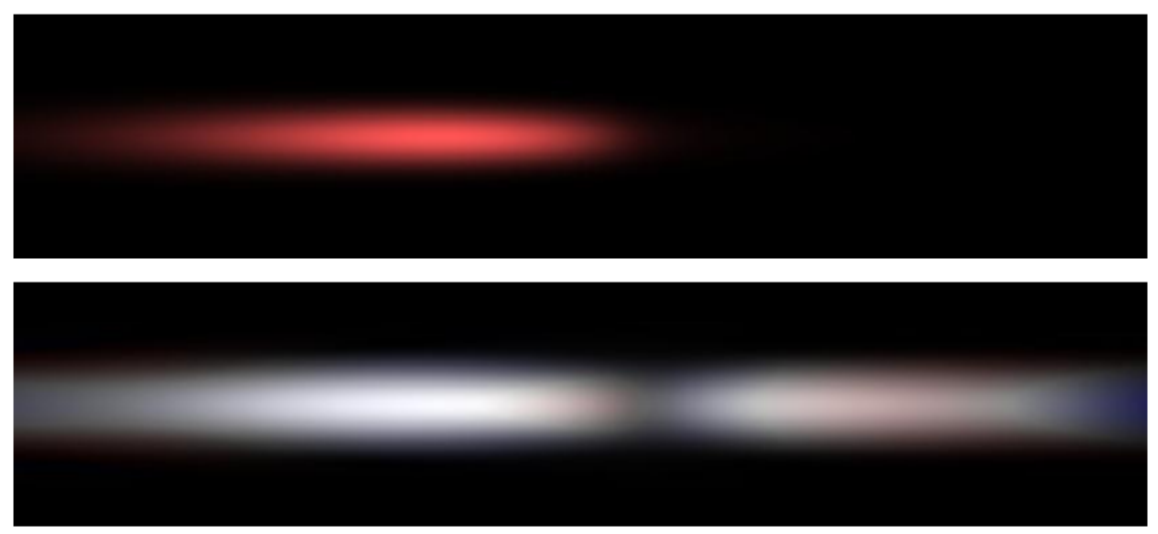

virtual waist plane

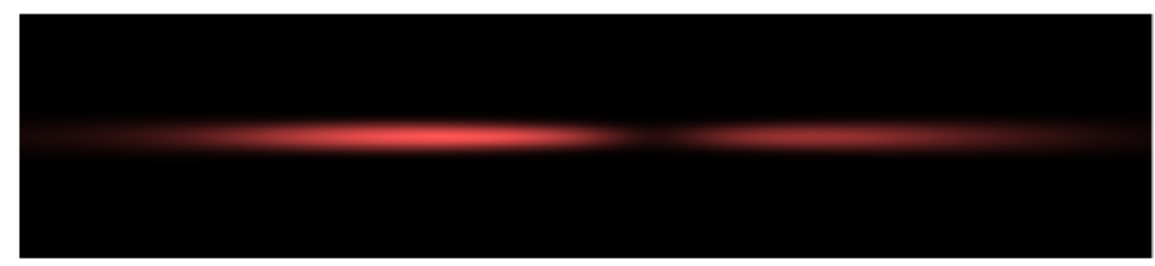

Figure 22. Grating and field images from the design with $150 \times 4000 \mu \mathrm{m}^{2}$ stripe size and 7-quantum regions

The overall field dimensions are $770 \mu \mathrm{m}$ by $9000 \mu \mathrm{m}$. The z-axis points to the right. The nearly colorless near field indicates good beam quality (phase-represented by color). $R(z)$ and $S(z)$ are the right- and left-propagating field slowly varying amplitude functions, respectively, 
Owing to the longer wavelength, the virtual waist size is roughly four times larger than with 1- $\mu$ m devices. The narrow 5-degree lateral far-field full width half maximum (FWHM) for the 4-mm length design is associated with its high aspect-ratio.

Thermal influences to the refractive index were not included. Their inclusion would slightly lower the beam quality but can be compensated by fine tuning higher-order terms in the grating phase expansion. Such refinements would be necessary after experimental devices and estimates of heat load based on experiment are available.

A stability test was performed on the $150 \times 4000 \mu \mathrm{m}^{2}$ design with the 7 -quantum region material design. The test consisted of incrementally increasing the antiguiding until the fields filamented. The antiguiding value reached $\alpha_{B}=4.5$ at filamentation, which is considerably higher than typical values measured in GaAs-based devices $\left(\alpha_{B} \approx 2\right)$. This numerical test result does not guarantee that the actual device will not filament but it does at least indicate that non-filamentation is possible. The lower sensitivity is likely due to the larger diffraction-limited spot (again due to wavelength scaling). The predicted $\sim 9 \%$ optical-to-optical efficiency is somewhat lower than that obtained in Reference 35. Two item underlie the low efficiency: non-radiative recombination (as parameterized by $\eta_{i}$ ) and losses from absorption, both in the metal and from free-carriers. The non-radiative recombination is a materials issue and will not be addressed here, although we predict there is some error in our gain prediction that will hopefully be refuted by experiment.

To see how different parameters affect the efficiency, a few trial cases were run. First the 5 quantum well $150 \times 4000 \mu \mathrm{m}^{2}$ (stronger grating) was run using the same $5 \mathrm{~cm}^{-1}$ absorption coefficient as in the 7 quantum-well $\sim 150 \times 4000 \mu \mathrm{m}^{2}$ modeling. This produced a DQE of 0.28 versus 0.20 with $10 \mathrm{~cm}^{-1}$ loss. This shows that grating strength is in part limiting the efficiency. To see an extreme case, grating and absorption parameters for a typical $980 \mathrm{~nm}$ SE-DFB device were run $\left(\alpha_{\text {surf }} \sim 50 \mathrm{~cm}^{-1}\right)$. This run produced a DQE of 0.45 - a very dramatic improvement. Yet the combined metal and free-carrier loss was $6 \mathrm{~cm}^{-1}$, actually larger than for the 7 quantum well $150 \times 4000 \mu \mathrm{m}^{2}$ case. Thus, it is clear that grating strength is in part limiting the efficiency, not just absorption. Conceptually, a stronger grating diffracts light out before it has propagated very far in the medium thereby avoiding loss. Ideally, we should have $\alpha_{\text {surf }} \gg \alpha_{\text {metal }}+\alpha_{f c}$, where $\alpha_{f c}$ is the free carrier loss.

But these observations are only parametric. The metal loss is expected to increase dramatically as the grating is made stronger, thus offsetting the gain from increased grating strength. Ideally, we would have grating that is both stronger $\left(\alpha_{\text {surf }} \sim 50 \mathrm{~cm}^{-1}\right)$, and has a very low loss $\left(<10 \mathrm{~cm}^{-1}\right)$. But given the optical properties of $\mathrm{Au}$ and $\mathrm{Ag}$ the simple etch-relief, metal-coat grating approach may never achieve this goal. This points out the need for a grating layer structure that could achieve sufficiently strong coupling. One thought is air-gap grating, which has a large index contrast

\subsection{Fabrication and Packaging}

\subsubsection{Fabrication Steps}

Figure 24 presents the proposed steps to fabricate the SE-DFB outlined and described herein:

initial epitaxy The epitaxy is grown by MBE on a GaSb substrate. This epitaxial structure is on the order of five microns thick, and includes an optical waveguide in the growth direction, acting as a slab 

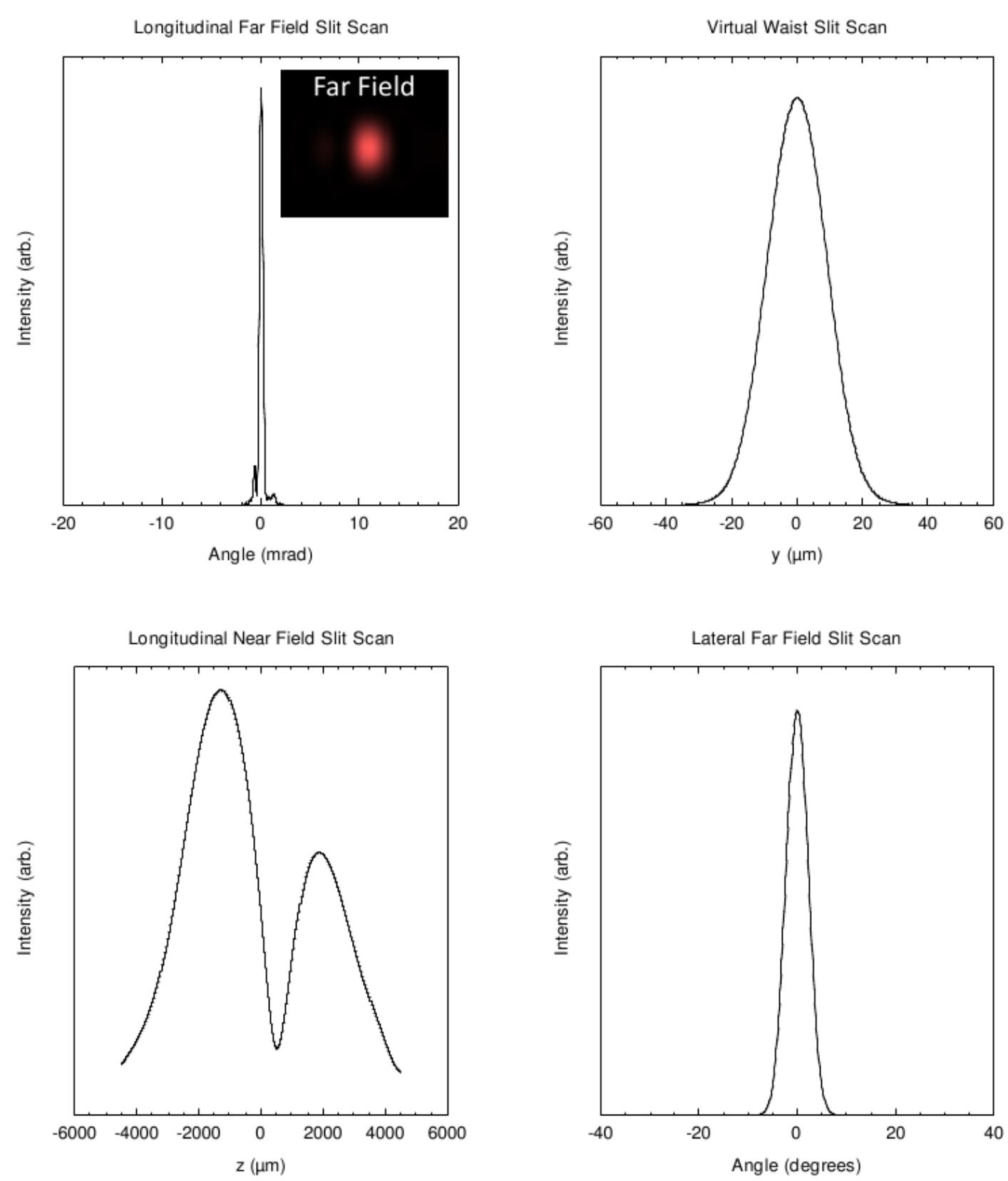

Figure 23. Simulated slit scans of the far field, near field and virtual waist Devices are the $150 \times 400 \mu \mathrm{m}^{2}$ design with and 7-quantum regions 


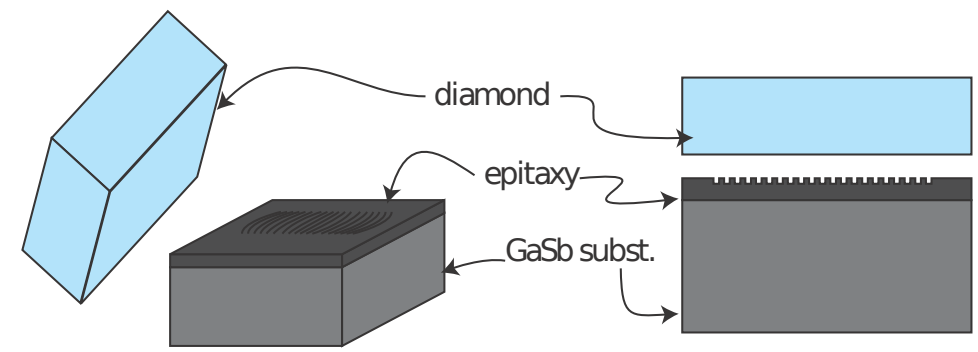

a)

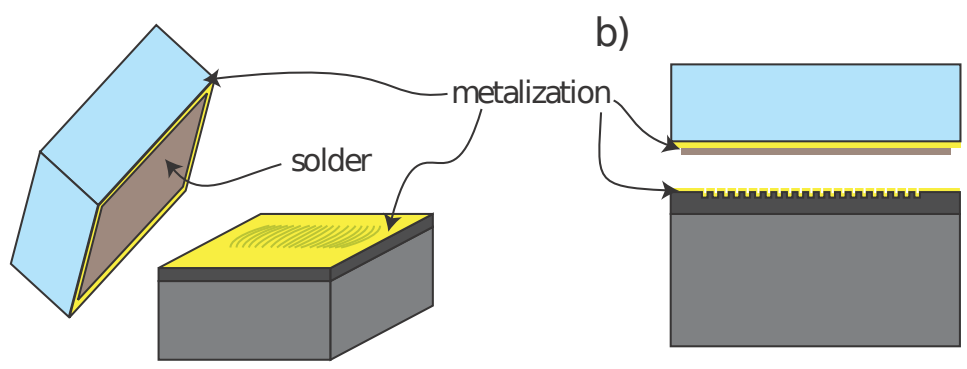

C)

d)

e)

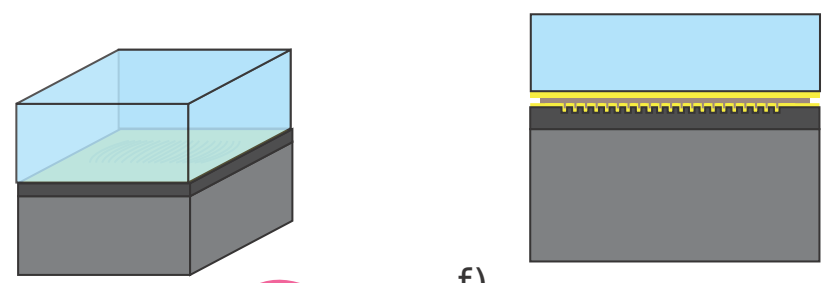

f)

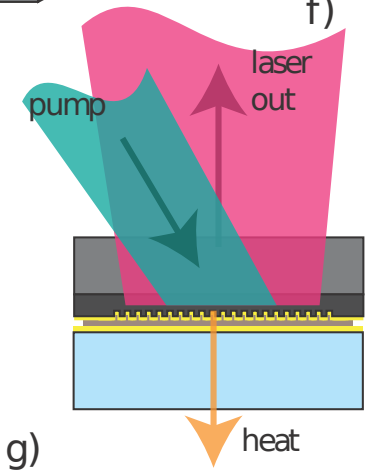

Figure 24. Proposed type-II SE-DFB processing steps.

$a, c, e)$ show 3-D renderings of structures, while b,d,f) show cross-sectional views. g) shows the notional embodiment of the final device under test. NOT TO SCALE. 
waveguide (no guiding in the lateral direction). Within the optical waveguide there are several (nominally five) type-II quantum wells. The optical core absorbs the pump laser frequency, and these excited carriers relax into the quantum wells, recombining and giving off stimulated emission.

patterning the grating Figure 24a,b shows the epitaxial structure on the GaSb substrate (gray) as well as a commensurate diamond structure (blue), which will facilitate heat spreading and removal under the laser. The first step is to create a surface-grating structure into the epitaxial structure. When the period is properly selected, this will provide feedback (from a "forward" to a "backward" traveling wave), as well as some portion emitting from the surface, and into the substrate. The "surface-emitting mode" will ultimately be evanescent, and all of the power will be emitted through the substrate. The mode interaction with the grating teeth (previously described in Reference 36) was previously calculated to necessitate grating teeth on the order of $0.4 \mu \mathrm{m}$ deep with a period slightly larger than $1 \mu \mathrm{m}$ and a nominal duty cycle of $50 \%$. While this feature size is large for electron-beam lithography, and small for photolithography, we have determined that at least the first experiments will rely on electron-beam lithography, owing to the flexibility of the technique, allowing for multiple periods and active control over the duty cycle. We chose to transfer the patterned e-beam resist into the semiconductor through dry-etching, relying on $\mathrm{BCl}_{2}: \mathrm{Cl}_{2}$ etch chemistry. This process yields smooth surfaces and vertical side-walls, and should be compatible with capably achieved in-house. While we have some previous unimpressive results[37], we think the ending results (with low $\mathrm{Cl}_{2}$ fraction) indicate a direction forward for patterning this grating structure.

metallizing the structure To assure the surface-emitting mode is suppressed (thus not wasting power), we may metallize the surface of the grating. Because this surface is interacting with the intra-cavity mode, and is distributed throughout the cavity, this metal surface has to be of high quality and purity.

Moreover, because the surface is patterned, the metal must coat conforming to the surface, making it within the teeth and coating the side-walls. We numerically investigated multiple metals, but either gold or silver were deemed to be similar to each other, and superior to other metals[36]. Figure $24 \mathrm{c}, \mathrm{d}$ shows both the epitaxial structure and the diamond heat-spreader coated with metal. To deposit the metal, we either rely on sputtering, or evaporation. The benefit of the sputtering is that it is known as a naturally conformal deposition technique, although the resulting metal quality is slightly lower than evaporation. Evaporation, on the other hand, is directional, and will not deposit conformally without some planetary motion of the substrate, varying the line-of-site from the deposition source (crucible). Whether the metal is gold or silver, a thin metallic blocking-layer should be put above the metal in contact with the semiconductor. The purpose of this layer (likely nickel) is to avoid an intermetallic forming from the solder layer (e.g.indium solder forms $\mathrm{InAu_{2 }}$ which is an optically lossy metal). Above this layer, we likely want a gold layer which would prohibit oxidation of the Ni layer, and provide a metal which indium will wet to.

The other step in this process is solder deposition. We have the option to use indium preforms, however we have been developing thin solder layers for the VECSEL project (see Section 6.2). Because this solder is within the thermal path for our lasers, and its thermal conductivity is low, thinner solder will improve device operation. A reasonable indium thickness for high power devices is around $5 \mu \mathrm{m}$, While it is easier to deposit thinner layers, they don't bond as well because of non-planarity of the diamond, and to a lesser extent, the semiconductor.

bonding the laser The laser is bonded near or slightly above the solder reflow temperature (in the case of indium, we often use $180^{\circ} \mathrm{C}$, though the reflow temperature is $157^{\circ} \mathrm{C}$ ). We most often perform this 
on a hot plate in our test lab using our bonding-control-unit . If more precise control of temperature or pressure is necessary, or relative positioning is essential, we can use out flip-chip bonder within the cleanroom.

final manifestation Figure $24 \mathrm{~g}$ shows a cross-section of the finalized structure. The pump $(\lambda \sim 2 \mu \mathrm{m})$ comes in at a slight angle relative to the substrate, which is transparent to the pump and the lasing wavelength. The pump light is absorbed in the waveguide core and excited carriers result in spontaneous and stimulated emission from the type-II wells. The stimulated emitted photons are guided and amplified in the optical waveguide, and fed back within this waveguide using the second order of the grating. A fraction of this light is diffracted normal to the substrate and is the laser output. The heat is removed from the "surface" of the epitaxial structure, which at this point is solder-bonded to a large thermal-conductivity diamond and subsequently removed through conduction cooling, or some other means.

\subsubsection{Grating Patterning}

As outlined in previous updates, the grating pitch for a second-order grating necessary to provide reflection and surface-emission is $\Lambda \sim 1.1 \mu \mathrm{m}$, and the duty cycle should be around $50 \%$. Features of this size lay between resolution typically achieved by the easy, and manufacturable photolithography, and the more difficult, although flexible electron-beam lithography.

While we discount this approach for development, selecting a more flexible approach such as electron-beam lithography, one technique that may be used for more manufacturable grating patterning may be proximity photolithography. Photolithography is the technique of choice for microfabrication, primarily due to ease, variety of photoresist, and ease of manufacturing, because wafers may be exposed en masse, instead of individual patterns written. While there are many different types of photolithography (contact, proximity, projection, deep-UV), to some extent, they are all limited to the resolution allowed by the incoherent, UV light utilized in the system. Mercury lamps are most typical and often have a filter about i-line $(\lambda=365 \mathrm{~nm})$ of mercury. This, combined by system optics, intimacy of mechanical contact of mask with the wafer, limits "manufacturing features" to about $0.9 \mu \mathrm{m}$ and "research features" to about $0.5 \mu \mathrm{m}$. For us, then, the features achievable by photolithography are very close to the necessary resolution to create our patterns, but because we have the advantage of a quasi-one-dimensional periodicity, we may rely on phenomena such as Talbot imaging to aid us in transferring photomask images into the photoresist. In truth, because of photoresist edge-bead effects, it is difficult to get true contact between the mask and the photoresist-covered wafer, so we have to be prepared to have some amount of air-gap between the two. Practically, if we can control the air gap distance (our Karl Suss MA6 has positioning accuracy of about $1 \mu \mathrm{m}$ ), proximity exposure is preferable because it minimizes any contact of the mask with surfaces that may result in mask feature degradation.

To analyze the proximity exposure, we use the scalar Rayleigh-Sommerfeld diffraction integral[38]. This approach allows for finding even near-field solutions to a thin mask, which includes evanescent fields. This is manifested in solutions to the following integral:

$$
U(x, y, z)=\frac{1}{2 \pi} \iint_{\text {aperture }} U\left(x^{\prime}, y^{\prime}, 0\right)\left[\frac{z}{R}(1-i k R) \frac{\exp ^{i k R}}{R^{2}}\right] d x^{\prime} d y^{\prime}
$$

where $U(x, y, 0)$ is the field immediately after the mask (incident field multiplied by the aperture function), $k$ is the wavenumber, and $R$ is the magnitude of the distance between any point $\left(x^{\prime}, y^{\prime}, 0\right)$ in the aperture and a 

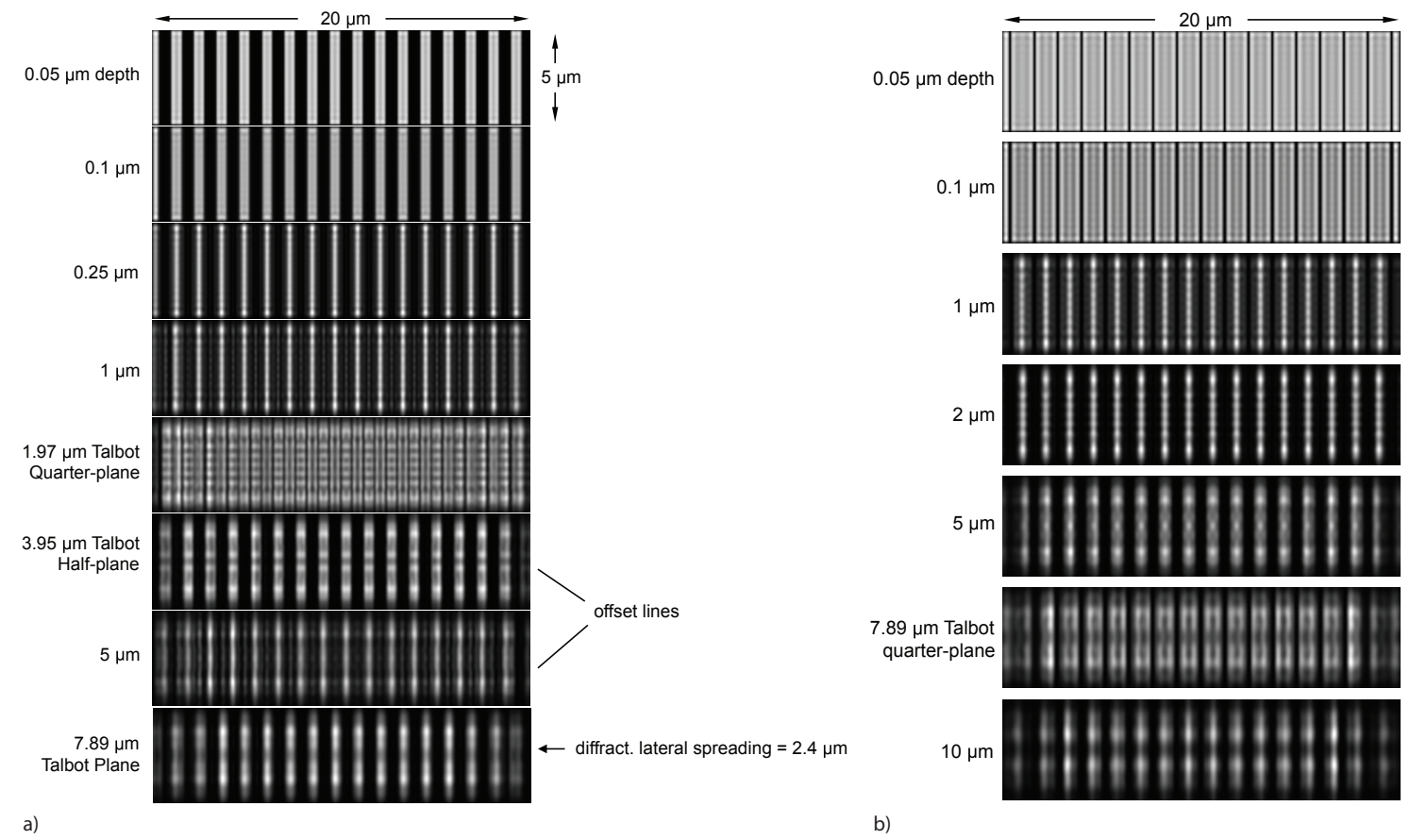

b)

Figure 25. Rayleigh-Sommerfeld diffraction intensity patterns for coherent proximity lithography

Computed Rayleigh-Sommerfeld diffraction intensity patterns of a) amplitude mask, and b) $\pi$-shift phase mask with a period of of $1.2 \mu \mathrm{m}$. The computation window is $20 \mu \mathrm{m} \times 5 \mu \mathrm{m}$, and the Talbot Plane is at $7.89 \mu \mathrm{m}$ below the mask, defined by the i-line illumination.

point in the final plane $(x, y, z)$. Equation 29 can be thought of as the Huygen's principal, wherein the first Rayleigh-Sommerfeld solution, which utilizes the Green's function $G(\vec{r})=\exp (i k R) / R-\exp \left(i k R^{\prime}\right) / R^{\prime}$. The radiation pattern associated with this source is given by:

$$
u_{p h}(x, y, z)=-\frac{1}{2 \pi} \frac{\partial}{\partial z}\left(\frac{e^{i k r}}{R}\right)=-\frac{1}{2 \pi}\left(i k-\frac{1}{R}\right) \frac{e^{i k R}}{r} \frac{z}{r}
$$

While this scalar solution is fairly straight forward, it still requires very large amount of computation, typically $\sim 10^{10}$ inner loop computations which grow as total number points squared.

We see in Figure 25a, which has a plane-wave illuminated amplitude grating (aperture is either 0 or 1), that this mask has about 1-micron of depth accuracy in tolerance that will reliably create the mask. In Figure 25b, the amplitude mask is replaced by an equivalent phase mask, where the aperture either transmits 1 or -1 . The latter example is more tolerant to depth errors, accuracy of about $4 \mu \mathrm{m}$ around $3 \mu \mathrm{m}$ depth. This depth of field increase is mainly due to the period doubling a result of the requirements of phase masks.

The features fade near the edges of the computation window. This fading, evident in Figure 25, is because Talbot theory relies on infinite periodicity. However our calculations don't assume reflective boundary conditions, the edge of the computation window is also the edge of the aperture. We will therefore see this in the actual mask patterns, although because they will be so close to the edge of the laser cavity that we believe this won't result in deleterious effects within the laser cavity. 


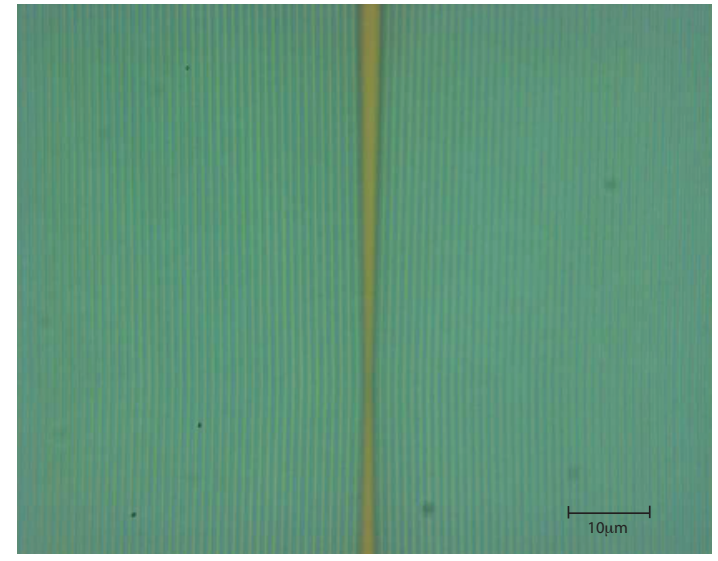

a)

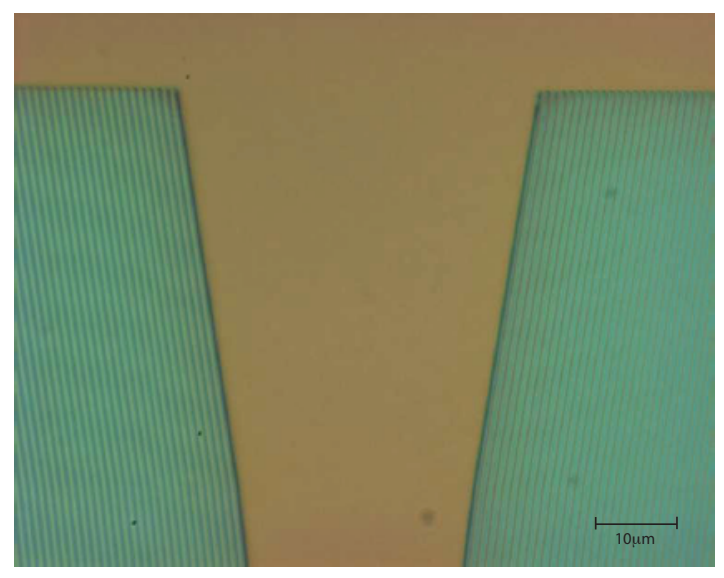

b)

\section{Figure 26. Microscope image of best exposure result of e-beam lithography}

Dose of $300 \mu \mathrm{C} / \mathrm{cm}^{2}$. At this rate, the approximate exposure of complete devices is just under 1 hour.

As an alternative, these patterns may be written by direct means like electron-beam lithography. This technique is best suited for small patterns of "reasonable" size $(100 \mu \mathrm{m} \times 100 \mu \mathrm{m})$, and critical dimensions in the range of $10 \mathrm{~nm}$. We don't question that this technique can achieve features of $0.6 \mu \mathrm{m}$, but the large laser dimensions may be complicated by the following:

stiching Because e-beam lithography is optimized for writing small features, the typical field of view is $500 \mu \mathrm{m} \times 500 \mu \mathrm{m}$. Because our lasers are larger (about $4 \mathrm{~mm} \times 1 \mathrm{~mm}$ ), this means we must stitch together several fields of view, keeping the grating in each field in phase relative to its nearest neighbors. This should be possible, although the mechanical move between fields has a certain amount of error associated with it that must be evaluated.

write time The large, high-density pattern means we run the risk of very long pattern write times. Moreover, because the grating pattern has curved lines, and the grating software breaks the software into Cartesian boxes, the curved lines may take longer to write.

Figure 26 shows our initial test pattern done to determine the approximate dose necessary to create the SE-DFB patterns we are after. ZEP-520A e-beam resist was spun onto GaSb substrate samples of $25 \mathrm{~mm}^{2}$, and then test patterns with doses of $200,300,400,450,500$, and $550 \mu \mathrm{C} / \mathrm{cm}^{2}$ were exposed. After developing in HR ZEP developer, the patterns were inspected with optical microscope. While doses of 200-450 $\mu \mathrm{C} / \mathrm{cm}^{2}$ were all acceptable, $300 \mu \mathrm{C} / \mathrm{cm}^{2}$ had the best fidelity, while 500 and $550 \mu \mathrm{C} / \mathrm{cm}^{2}$ patterns had sufficient undercutting, a result from back-scattered electrons, which caused large portions of the patterns to be washed away. This calibration was necessary for the approximate patterns we plan on using, but because different substrates have different electron back-scatter patterns, this calibration was essential. Moreover, we are pleased to now know that each pattern approximate write time is about 1 hour, which is actually significantly less than feared.

The next step is to transfer these patterns into the wafer using inductively-coupled plasma reactive ion etch (ICP). Progress on this effort is reviewed in Section 4.4.3. 


\subsubsection{Dry Etching GaSb Materials}

To remind the reader, for the two designs found thus far, we would like to etch grating teeth (each being approximately $600 \mathrm{~nm}$ wide) down into GaSb about $400 \mathrm{~nm}$. For a controllable an repeatable etch, we would like this etch to be 1-3 minutes, resulting in an etch rate of nominally $0.15 \mu \mathrm{m} / \mathrm{min}$. As evidenced by Figure 27, we have taken a typical dry etch (begun from an existing recipe in our chamber). While the etch-rate is quite fast, the fractional roughness (RMS surface roughness, as determined by AFM, as a fraction of the etch depth) is very large. The resulting etch surface roughness is within the optical cavity and will act as scattering centers which create a loss-term, as well as deteriorate the beam resulting laser quality. We perimetrically changed the chamber pressure, as it also imparts characteristic features controlling the etch rate and roughness. At low chamber pressures, there are insufficient chemicals to etch, so the etch rate drops. At large chamber pressures, the reactive species mean-free path is too short, and etch rates again suffer. Between these extremes, there is a maximum etch rate. Because below this maximum point, etching is often ballistic (physical), the surfaces can be quite rough, and the fractional roughness generally drops off at larger chamber pressures because there are sufficient surface interaction times to allow for chemical "clean-up". Perhaps we see these features in Figure 27, but it is difficult to discern. The etch rate peak may be likely found at $40 \mathrm{mT}$, but the roughness does not drop as dramatically as one may expect.

Follow-ons to this work indicate that lower fractions of $\mathrm{BCl}_{3}$ gas (including no $\mathrm{BCl}_{3}$ ) have shown much more promising results. While the etch-rate drops, so does the surface roughness.

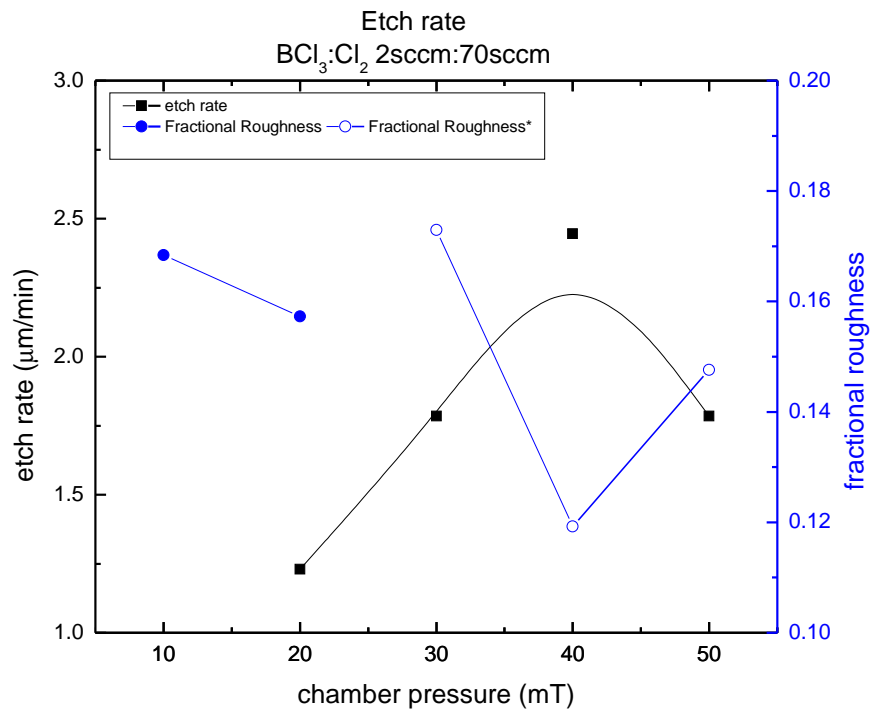

Figure 27. Etch-rates and etch roughnesses of GaSb etched using ICP

Recipe using $\mathrm{BCl}_{3}: \mathrm{Cl}_{2}$ etch. This recipe has control over the etch-rate, although the roughness variation is inadequate for a sufficient grating etch. The unfilled data indicates approximate values. 


\subsection{BROADBAND NONLINEAR COMPONENTS}

Currently VECSELs employ semiconductor-based saturable absorbers which have a narrow tuning range and require complex fabrication procedures. By developing a graphene-based saturable absorber, one can take advantage of its zero bandgap structure and therefore its frequency-independent absorption as well as its thermal and mechanical stability to passively mode-lock lasers over a wide frequency range - potentially through the terahertz regime. Here we report on recent developments in graphene mode-locking of VECSELs, specifically for high power operation. Further, we present work done on the nonlinear optical properties of graphene, specifically pertaining to the development of saturable absorbers as well as optical limiters.

From a practical standpoint, semiconductor-based saturable absorbers require complex fabrication techniques, careful band engineering and have a very narrow bandwidth. To overcome these issues graphene has emerged as a promising saturable absorber material to mode-lock lasers. Although significant work has been done to integrate graphene into fiber and solid state lasers, there has been limited headway in integrating graphene in a VECSEL due the cavity's sensitivity to losses. [39-44]

Single layer graphene consists of a layer of hexagonally packed carbon atoms, $0.3 \mathrm{~nm}$ thick with a linear absorption of $2.3 \%$.[45, 46] This single layer of carbon shows a nearly featureless absorption spectrum from the visible to the Terahertz due to its zero-bandgap and his linear dispersion which thereby allows intraband transitions at potentially all wavelengths These carriers once excited, thermalize extremely fast (in the order of tens of femtoseconds) to the bottom of the band thus preventing additional absorption. Hence when irradiated with high fluences graphene undergoes an optically induced transparency which aids in the creation of ultrashort pulses when integrated into a laser cavity. On the other hand, functionalized graphene-based materials display broadband optical limiting due to two-photon absorption (TPA), reverse saturable absorption (RSA) and nonlinear scattering (NLS).[47-51] Suppression of the output fluence has been shown mostly in graphene-based solutions which allow the possibility of a strong nonlinear component due to scattering. However since such materials in suspension form are not practical components for to integrate into a structure the challenge has been to develop graphene films in solid matrices [52] which are better suited for a device platform.

Over the last decade, graphene has emerged as an ideal material in optoelectronic devices ranging from saturable absorbers for mode-locking lasers to optical modulators and photo-detectors.[46, 53-55] Further graphene-based materials exhibiting tunable system parameters have been shown to exhibit enhanced electrical and thermal conductivity, mechanical stability and strong optical phenomena. A graphene-based material will hence be especially attractive as a saturable absorber in VECSELs due to its fast relaxation times, and strong nonlinearity over a wide wavelength range potentially from visible to the terahertz regime. However since a single layer of graphene has a significantly high absorption when taking into account its thickness its integration into a VECSEL cavity which is unable to sustain high loss has been a challenge. Broadband optical limiters on the other hand would be able to suppress high fluences at various wavelengths while being optically transparent. Optical limiting materials are extremely useful for systems vulnerable to laser damage such as sensors, eye protection etc. In this effort we therefore aim to study various graphene materials which exhibit a strong nonlinear optical response together with thermal stability. The aim is to try and manipulate these remarkable optical properties to realize nonlinear devices, particularly saturable absorbers and optical limiters. 


\subsection{Graphene Polymer Composites}

An overview of the optimized processing techniques employed to obtain a functionalized graphene solution and nanocomposite are described. In addition, we report on preliminary optical studies on various graphene-based samples. The samples discussed here consist of a water-based graphene solution, graphene-polymer composite solution and thin films. Dependence of factors such as concentration, deposition techniques and surface quality on linear and nonlinear optical properties are studied. Furthermore we demonstrate the ability to tune and/or control linear and nonlinear optical properties by varying concentration, host matrix and path length.

\subsubsection{Preparation Techniques}

Our first step was to create a graphene-polymer nanocomposite. This entailed optimizing processing techniques to ensure complete solubility of graphene in water. A schematic outlining preparation steps is shown in Figure 28. The first step was to dissolve a dispersant solution SDS in water. Graphene was then added and the solution was further sonicated until a visibly dissolved solution was obtained. Two concentrations of graphene in SDS-water are studied (a) $1.7 \mathrm{mg} / \mathrm{ml}$ and (b) $6.4 \mathrm{mg} / \mathrm{ml}$. Solubility was confirmed when the graphene did not settle at the bottom of the container for weeks.

Simultaneously, the polymer solution was prepared by dissolving PVA in water at a concentration of $24 \mathrm{mg} / \mathrm{ml}$. We then prepared various solutions with different concentrations of graphene in the PVA solution. As a starting point three solutions were prepared where the ratio of the graphene-SDS solution to the PVA solution varied. This was done to study the effect of graphene concentration on the linear and nonlinear optical properties of the composite. In addition to the solutions, spin coated and dropcoated samples were also prepared using the graphene-polymer composite solutions. These were spin/drop coated on a substrate and heated at $100^{\circ} \mathrm{C}$ for ten minutes to evaporate any remaining solvent. Furthermore a graphene-oxide solution was also prepared by dissolving graphene oxide in an SDS-water solution at a concentration of $0.8 \mathrm{mg} / \mathrm{ml}$. The graphene-oxide due to its -O- bonds dissolved in the water fairly readily[56, 57].
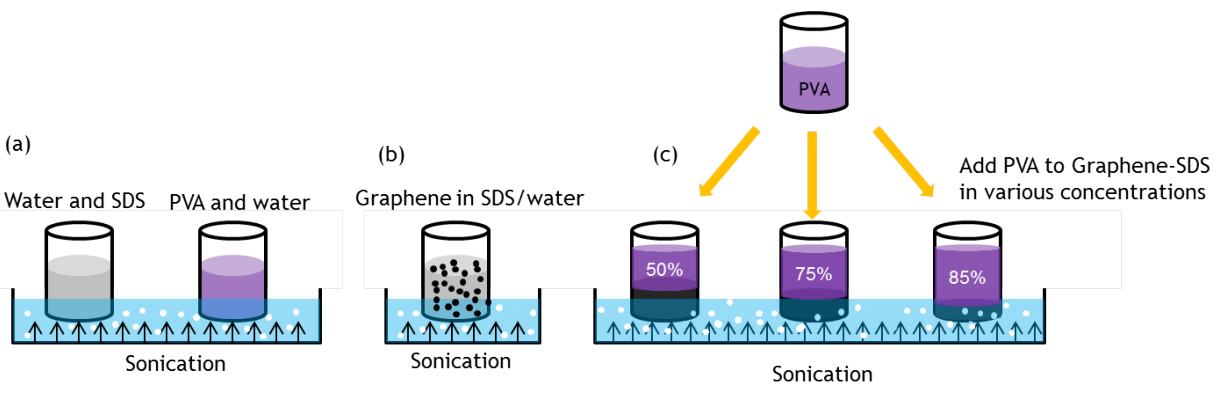

Figure 28. Schematic showing preparation of the graphene-PVA composites. (a) Two separate solutions of SDS in water and PVA in water are prepared, (b) graphene is added to the SDS/water mixture and sonicated (c) PVA is then added to the graphene-SDS/water mixture in various amounts to produce three different graphene-PVA concentrations. 

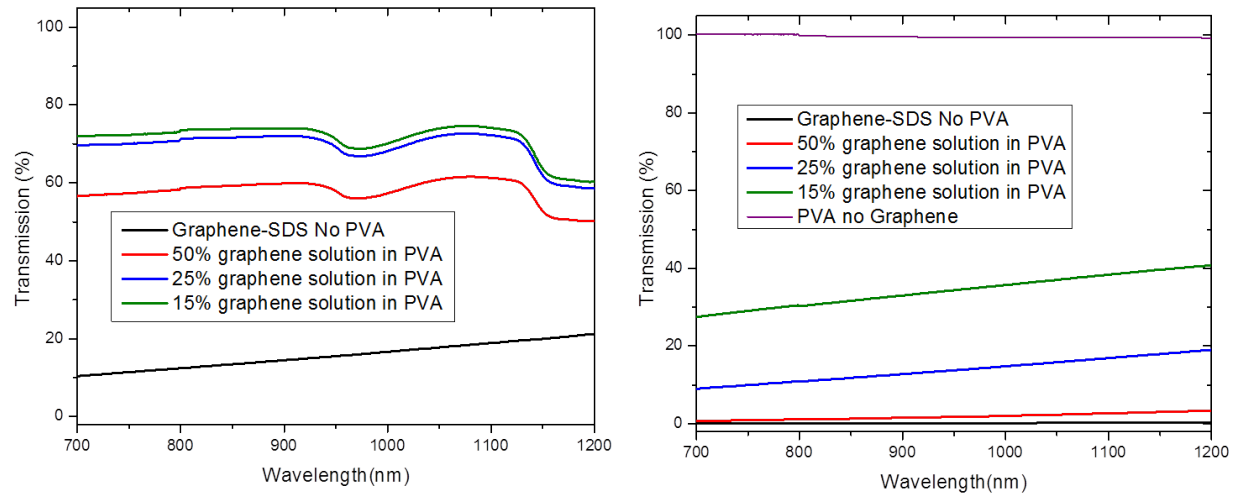

\section{Figure 29. Optical transmission of graphene-based solutions}

Graphene-SDS concentration of (a) $1.7 \mathrm{mg} / \mathrm{ml}$ in $5 \mathrm{~mm}$ path length cuvettes and (b) $6.4 \mathrm{mg} / \mathrm{ml}$ in $2 \mathrm{~mm}$ path length cuvettes. It can be seen that by increasing the concentration of graphene in our solutions we can tune the transmission of our samples.

\subsubsection{Graphene Optical Characterization Methods}

Transmission measurements are first carried out on the graphene-based solutions. The measurements were carried out using $5 \mathrm{~mm}$ and $2 \mathrm{~mm}$ path length cuvettes and are shown in Figures 29a and 29b respectively. Due to graphene's universal absorption $[46,57,58]$ we can see that over a wide range both the spectra of graphene composites remain fairly featureless. Also, as expected we observe that as the amount of graphene increases, so does the absorption. Therefore by increasing the concentration of graphene we can manipulate the linear transmission of our samples.

Solid samples were also prepared by spin coating the graphene-PVA composites on a GaAs substrate. The
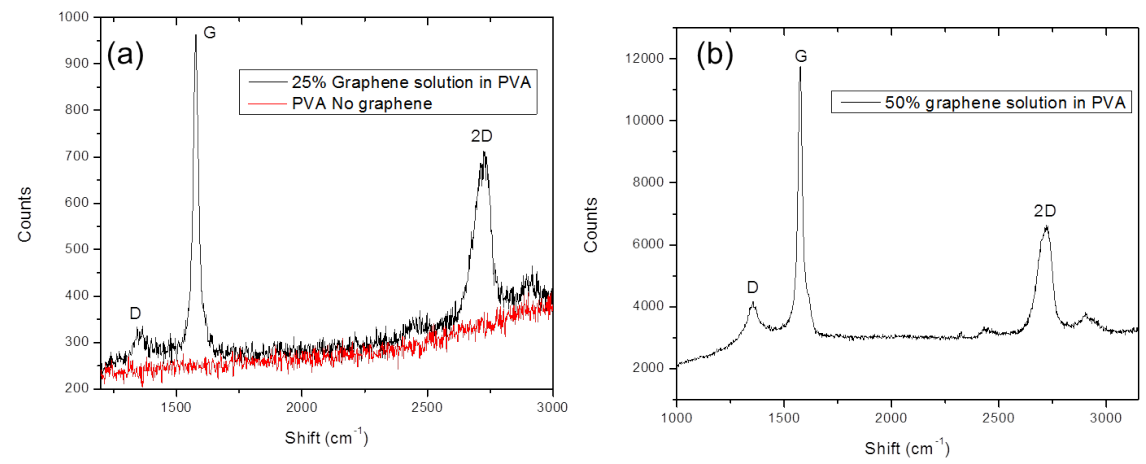

Figure 30. Raman spectra of graphene composite films

Raman spectra of (a) 25\% graphene-SDS solution in PVA (black) and PVA with no graphene (red) and (b) 50\% graphene-SDS solution in PVA (black) is shown. For both samples we can deduce good surface quality due to the low intensity of the D peak 


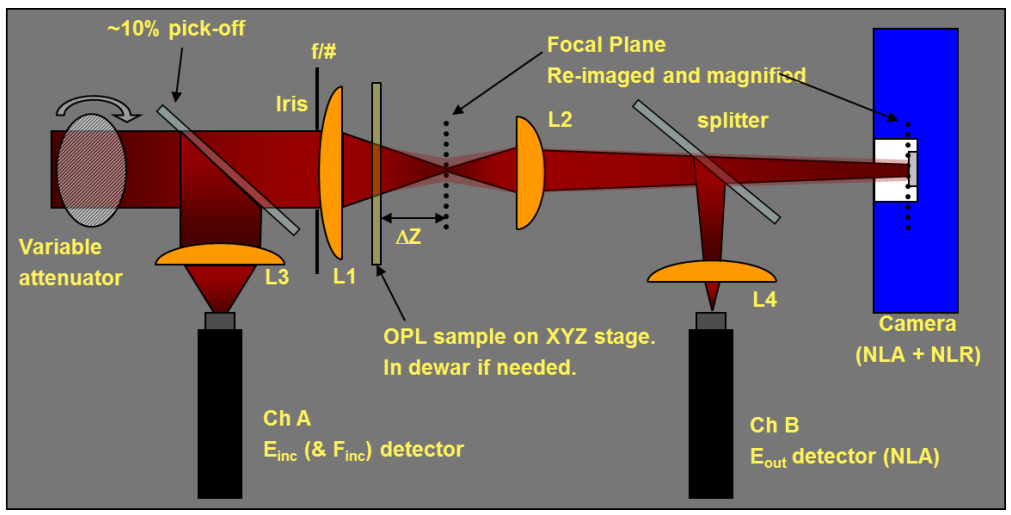

\section{Figure 31. I-scan measurement technique schematic}

Method used to conduct optical limiting experiments. Unlike conventional z-scan method, here the sample remains fixed and intensity is changed via a variable attenuator.

quality of graphene deposition on these were studied by conducting Raman measurements at $488 \mathrm{~nm}$ excitation and $50 \mathrm{X}$ objective. The Raman spectra is shown in Figures 30a and 30b. As expected for carbon based materials there exist three prominent peaks $[59,60]$. The Raman spectra of graphene-based materials includes a $D$ peak (absent from defect-free graphene), the $G$ peak located at $\sim 1580 \mathrm{~cm}^{-1}$ and the 2D peak observed $\sim 2700 \mathrm{~cm}^{-1}$. The presence of a $\mathrm{D}$ peak (occurring due to first-order zone boundary phonons) indicates a disordered carbon lattice due to structural defects, amorphous carbon or edge effects. The $\mathrm{G}$ band results from the in-plane vibration of carbon atoms and is the most prominent feature of graphitic material. The lineshape of the 2D peak (second-order zone boundary phonons) as well as its intensity relative to the $\mathrm{G}$ peak can be used to characterize the number of layers ' $n$ ' of graphene for $n<6$ [57]. Single layer graphene is usually characterized by a sharp, symmetric Lorentzian peak and a FWHM $<30 \mathrm{~cm}^{-1}$. As the number of layers increase the $2 \mathrm{D}$ peak becomes broader, less symmetric and decreases with intensity. Multilayer graphene samples are characterized by a multiple Lorentzian fit and a FWHM $>50 \mathrm{~cm}^{-1}$ [61].

For these samples we see that the D peak is significantly lower than the G peak thereby indicating very good surface quality. In Figure 30a we see the Raman spectra of a spin-coated PVA film (red) which unlike the graphene-PVA films shows no prominent features. The FWHM of the 2D peaks in both Figures 30a and 30b are $>50 \mathrm{~cm}^{-1}$ indicating multiple layer graphene. Although the films visibly look fairly uniform it should be noted that graphene is not evenly distributed across the substrate and such a Raman spectra attained from very localized regions with a noticeable cluster of graphene flakes.

With the development of well dissolved graphene solution we then carried out nonlinear optical measurements to study optical limiting mechanisms in the graphene samples in collaboration with AFRL/RXPJ. An optical limiter ideally should attenuate intense radiation while exhibiting high-transmittance for low-intensity light. Graphene-based dispersions have been shown to exhibit both saturable absorption (SA) and RSA . Each of these behaviors manifests itself depending on the environment, material properties and operation regime. Carbon and graphene based samples have been reported to show strong nonlinear optical limiting effects in the nanosecond and picosecond regime [48, 49, 51, 62, 63]. Such nonlinear behavior has been attributed to nonlinear absorption (NLA), nonlinear refraction (NLR), thermally induced nonlinear scattering, creation of localized excited states...etc.[51, 64]. The optical limiting measurements here were conducted on a setup whose schematic is shown in Figure 31. The setup is capable 

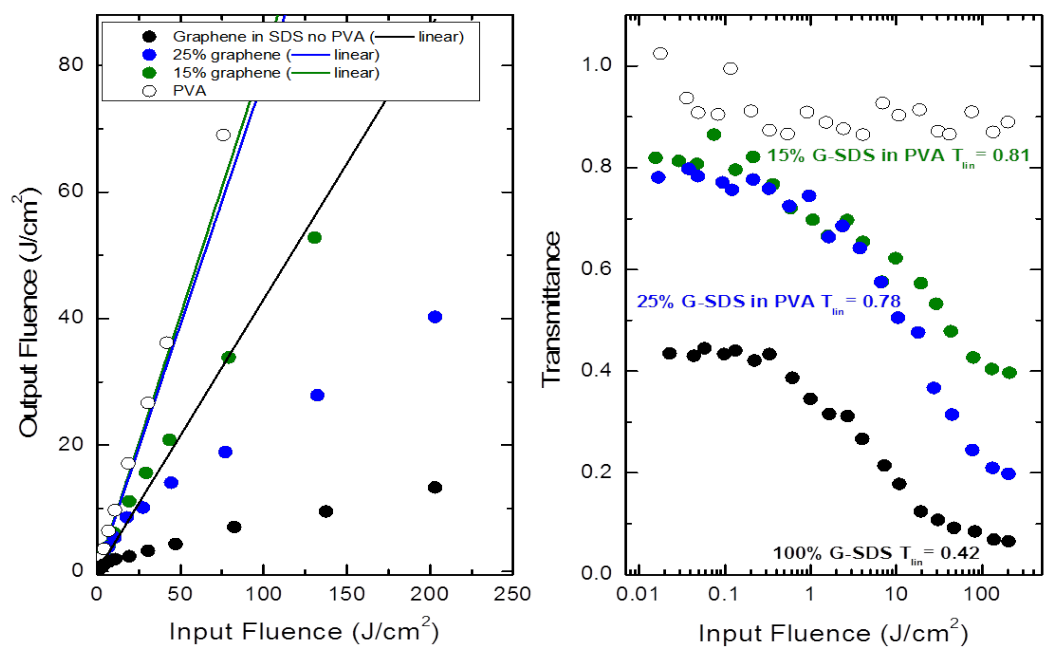

\section{Figure 32. Plots showing optical limiting in the dispersions developed using $1.7 \mathrm{mg} / \mathrm{ml}$ graphene-SDS solution.}

The plots (a) showing optical limiting behavior whereby output fluence decreases with an increasing amount of graphene (b) plot of transmission versus input fluence which shows that although the onset of limiting is comparable in all three solutions, the input threshold (point where transmission drops to have its original value) is the smallest for the solution containing the highest concentration of graphene.

of measuring NLA and NLR simultaneously. The fluence out is measured via an imaging system which also records the total transmitted energy. The fluence-in and incident energy is measured with another silicon meter as shown in the figure. The fluence-in is directly proportional to the measured incident energy. These nonlinear measurements were carried out at $785 \mathrm{~nm}$ wtih pulse duration $5 \mathrm{~ns}$. The sample is kept fixed while an attenuator is used to vary the input fluence. The first step of measurements were conducted on the graphene-based samples with concentration of $1.7 \mathrm{mg} / \mathrm{ml}$ in a $2 \mathrm{~mm}$ path length cuvette. The optical limiting curves are shown in Figures 32a and 32b.

In Figure 32a, a plot of output fluence versus input fluence for the graphene solutions are shown. We can see that as the percentage of the graphene solution is increased the output fluence decreases significantly. Figure $32 \mathrm{~b}$ shows a plot of the transmittance versus the input fluence. Here we notice that the onset of limiting (point where transmission starts reducing) is fairly comparable in all three solutions. This led us to believe that the onset of limiting is independent of the concentration. However the input threshold (where transmission drops to $50 \%$ of its initial value, or $3-\mathrm{dB}$ ) shifts to lower values with increasing graphene concentration. This is because higher concentrations of graphene are able to block the light more effectively at higher incident fluences than those with lower concentration. For example, the onset of limiting in all three plots is seen to be approximately $0.4 \mathrm{~J} / \mathrm{cm}^{2}$. However the graphene-SDS solution which contains no PVA and therefore the highest amount of graphene has the lowest threshold fluence of $6 \mathrm{~J} / \mathrm{cm}^{2}$ for which the transmittance drops to its initial value.

Attempts to tune the threshold value as well mimic observed trends, we conducted the same experiment with the higher graphene-SDS $(6.4 \mathrm{mg} / \mathrm{ml})$ solution. Similar data as before is shown in Figures 33a-c. As before 
we are able to reduce the transmittance considerably by increasing the graphene concentration shown in Figure 33a. In Figures 33b and 33c we plot the transmittance versus the input fluence. Here we notice that with increasing concentration the input threshold increases. This result is contradictory to those attained above and therefore further investigations need to be carried out to understand the dependence of concentration on the optical limiting mechanism. It should be noticed in Figure 33c that for the higher concentrated solution the transmittance slightly increases as the input fluence increases up to around $10 \mathrm{~J} / \mathrm{cm}^{2}$ which we believe to be saturable absorption. This may play a part in causing the deviation in these samples from expected results.

To further demonstrate our ability to tune the nonlinear behavior in the graphene dispersions we conducted nonlinear experiments on one of the graphene dispersions (25\% graphene solution in PVA) using cuvettes with different path lengths. By decreasing the path length of the cuvettes we reduce the effective length of our samples and therefore increased the output fluence thereby shifting the input threshold value. In Figure 34a the linear transmittance for all three samples is shown. As expected, we get a higher transmittance with a shorter path length. The data exhibiting the output fluence and transmission versus input fluence is shown Figure 34b and 34c respectively. The data shown Figure 34b is as expected - as the effective length of the sample decreases the output fluence increases. In Figure 34 (c) we see that as before the onset of limiting is not significantly affected by path length however the input threshold changes. For a greater path length we
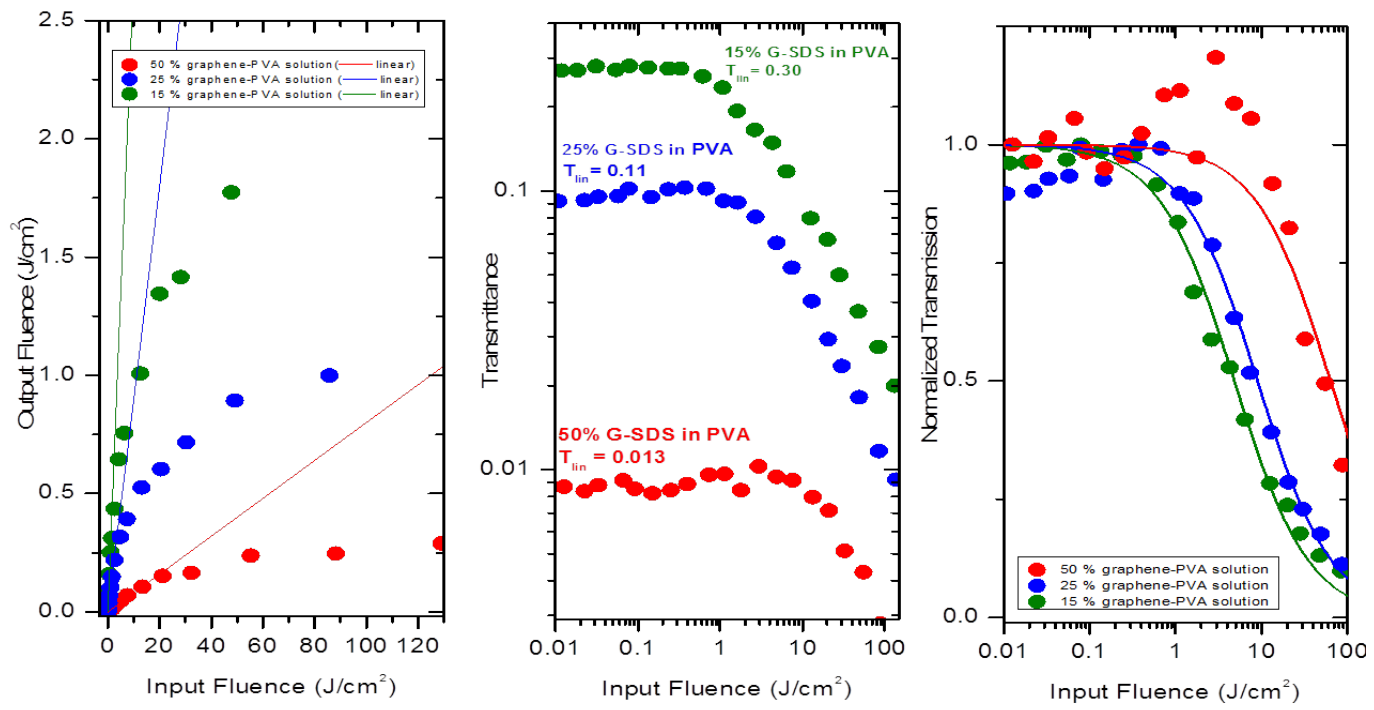

\section{Figure 33. Plots showing optical limiting in dispersions}

Power-dependent transmission using the higher concentration, $6.4 \mathrm{mg} / \mathrm{ml}$ graphene-SDS solution. The plots (a) showing optical limiting behavior whereby output fluence increases with decreasing amount of graphene (b), (c) Shows change in the limiting threshold whereby it shifts to higher fluences for increasing concentration but as before the onset of limiting remains fairly constant in all three samples. For the higher concentrations (25\% (blue) and 50\%(red)) graphene-PVA solution it should be noted that there is a slight increase in transmittance initially with increasing input fluence which is believed to be saturable absorption. 

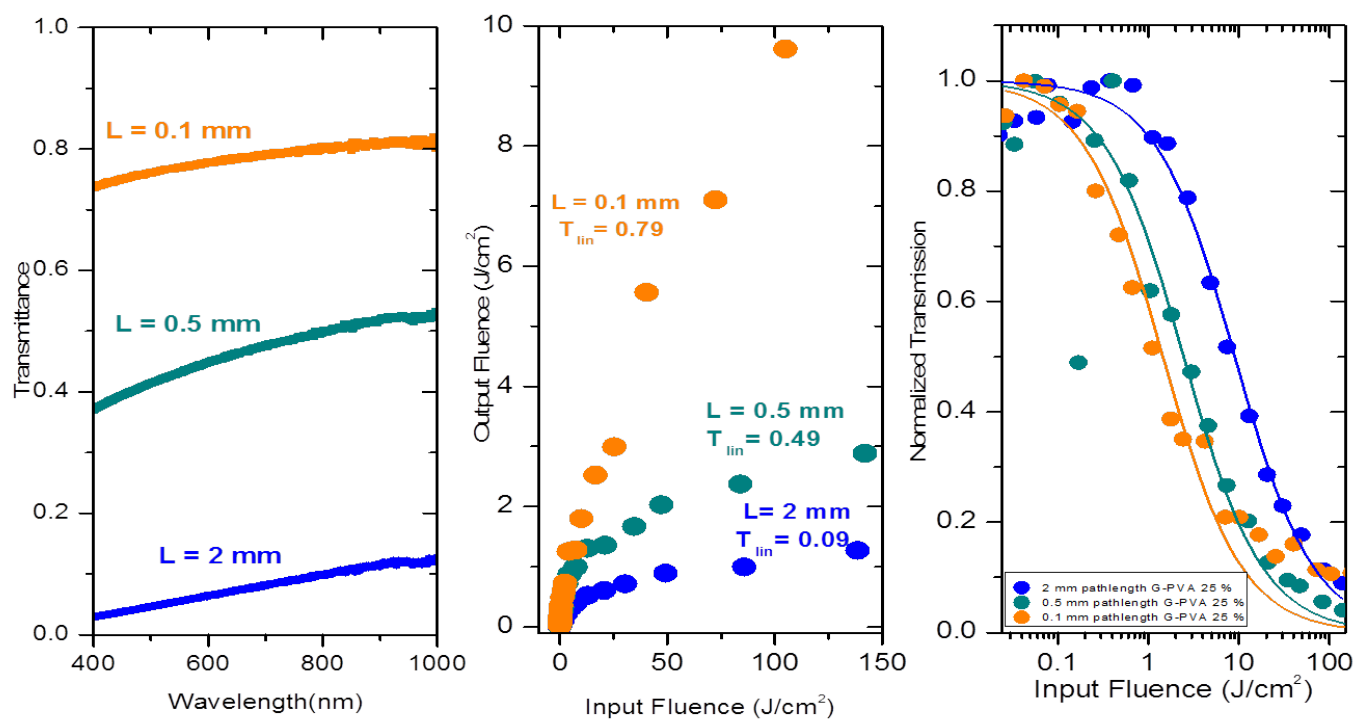

\section{Figure 34. Evidence of variable optical limiting behavior based on path length}

The plots (a) shows change in optical limiting behavior whereby with with smaller path-lengths we can increase the output and $(b)$ the input fluence threshold shifts to higher values with decreasing path length.

observe a higher input fluence which conceptually was unexpected. Therefore further studies are required to understand the unusual behavior of these systems.

Although it is clear from all the plots discussed above that we can tune the nonlinear response from the graphene-based samples the reasons for the nonlinear response are still not understood. To better deduce the reasons for the nonlinear behavior we analyze the energy and fluence transmission in one of our samples in order to analyze the contribution from NLR. This plot is shown in Figure 35. NLR can cause the fluence output to lower out (self focusing) or raise (defocusing). In Figure 35 the fluence is slightly raised than the energy transmission indicating some self focusing by the sample. However since the difference between these is not significant we can deduce that the response is due to NLA and any contribution from NLR is small. Other physical mechanisms can also lead to limiting behavior. For example since our solvent choice if water which has a lower boiling point (relative to other solvents used for such purposes)[51] we can attribute part of the nonlinear behavior to the creation of microbubbles and microplasmas. These are formed by rapid localized heating (due to strong linear absorption) giving rise to solvent bubbles which scatter the incident light causing a reduction in transmission. Other possibilities include the creation of excited states (under excitation) possessing higher absorption cross-section area than the ground state absorption cross-section area thereby leading to RSA.

The above optical limiting measurements were then repeated using an excitation wavelength of $1064 \mathrm{~nm}$ with a 16.7 picosecond pulse width. A comparison between the NL response of the $785 \mathrm{~nm}$ ( $5 \mathrm{~ns}$ pulse width) and $1064 \mathrm{~nm}$ (16.7 ps pulsewidth) is shown in Figures 36a and 36b. Here we see that optical limiting is sensitive to laser pulse width. In addition it shows the broadband response of graphene-based systems. Figure 36a shows the fluence dependence effect with the transmission versus irradiance plot. Here we see that at the 


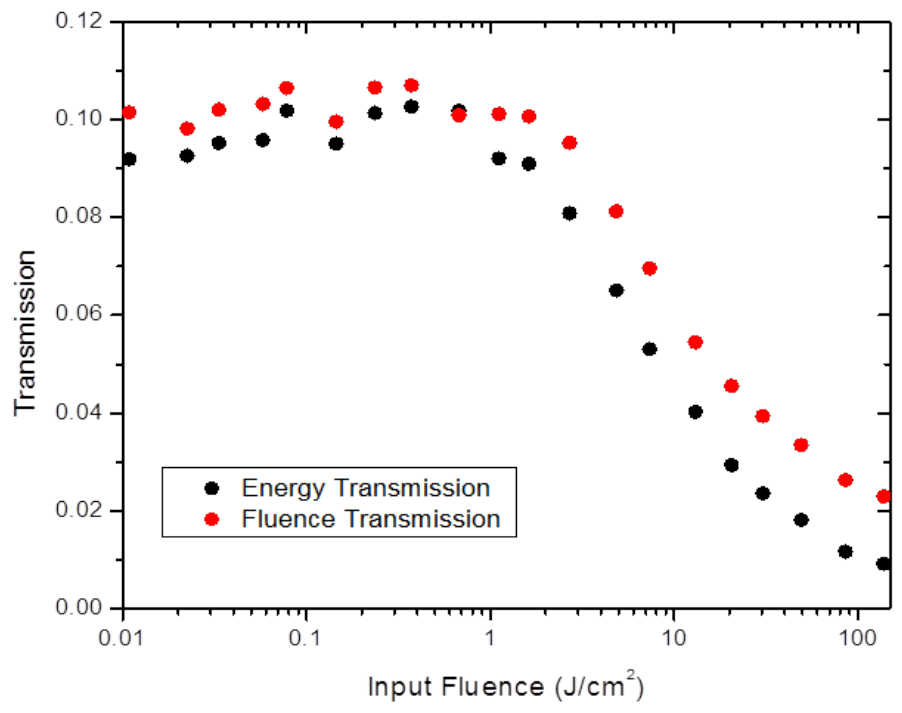

Figure 35. The energy transmission and fluence out is plotted versus input fluence The raised fluence out indicates positive NLR (selffocusing) -however as the difference between the energy transmitted and fluence transmission is not drastically different we can attribute the nonlinear response to NLA with very little contribution from NLR.
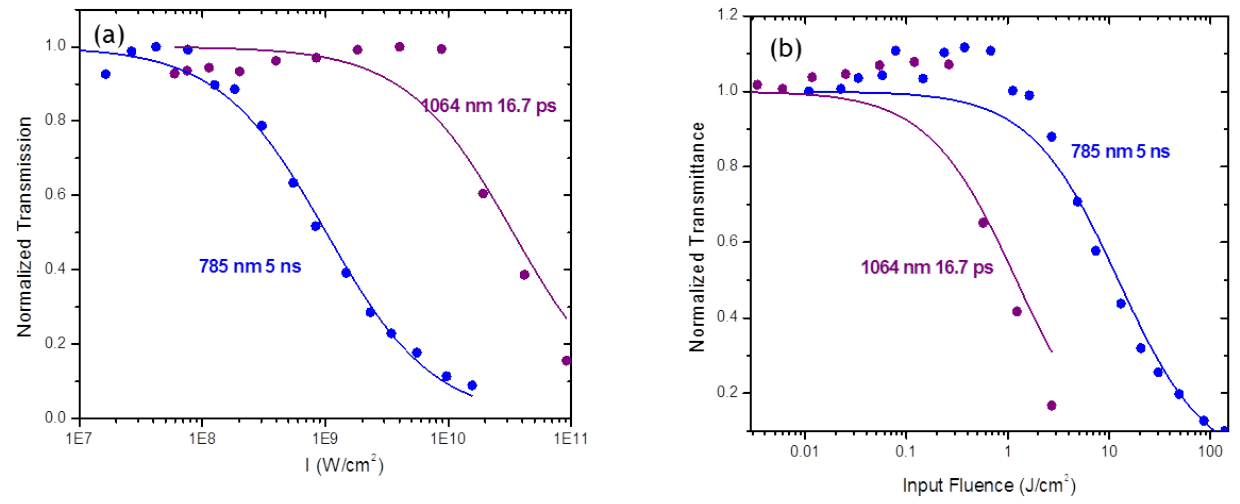

Figure 36. The energy transmission for various pulse-widths and wavelengths

(a) normalized transmission of ps-scale pulse at $1064 \mathrm{~nm}$ compared to $\mathrm{ns}$-scale pulses at $785 \mathrm{~nm}$ plotted as a function of peak irradiance. (b) same data as (a), but plotted as a function of pulse energy density (fluence). 

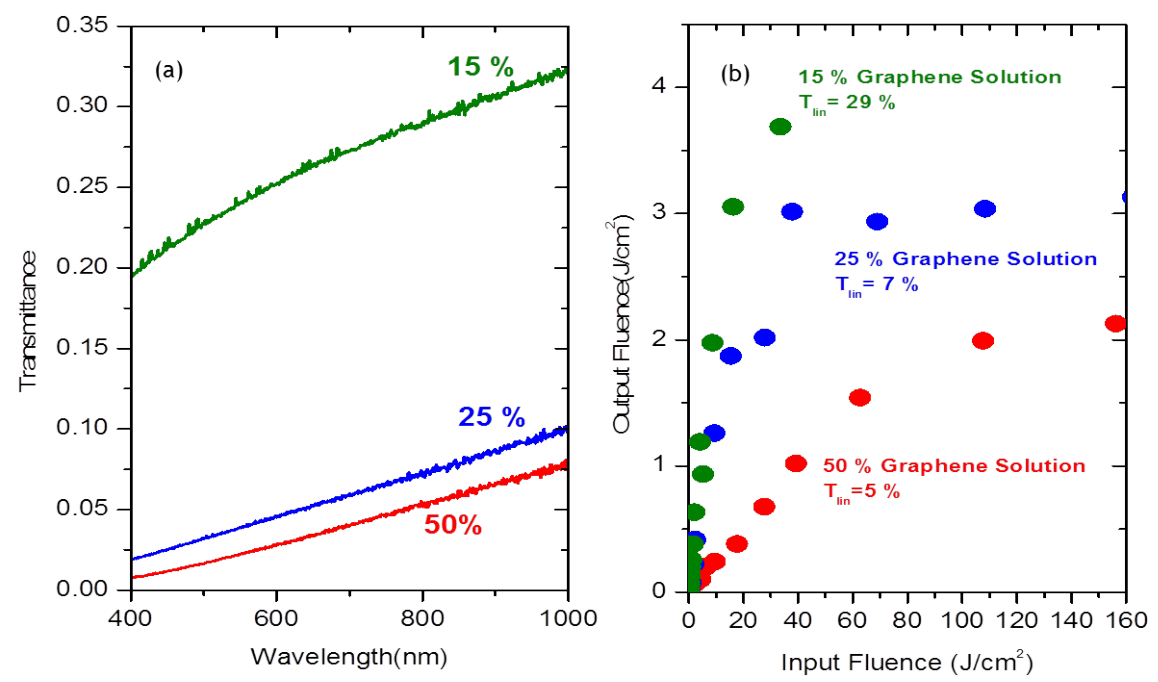

\section{Figure 37. Concentration dependance of linear and nonlinear material transmittance in films}

(a) linear transmittance of various concentrations of graphene composite. (b) nonlinear transmission of same films showing the concentration dependance of nonlinear effects.

same irradiance the longer pulse has a higher nonlinearity indicating that our system is fluence dependent. It is reported that in the nanosecond regime the parameters describing the thermal properties are more dominant in contributing to optical limiting. Therefore in the nanosecond regime the mechanisms contributing to the higher nonlinear behavior are thought to be due to nonlinear scattering caused by the formation of microbubbles and microplasmas. However in Figure 36b we observe an irradiance dependence indicating that sample shows higher nonlinearity for the shorter pulse (higher irradiance). This in the picosecond regime is attributed to nonlinear absorption (NLA) mechanisms. Therefore the nonlinear processes occurring here are both fluence and irradiance driven and further investigations are underway to understand this behavior fully[65].

We now extend our experiments onto solid samples. Three dropcoated samples were prepared using the concentrations described above. Once the substrate was dropcoated the sample was annealed for 10 minutes at $100^{\circ} \mathrm{C}$. Their limiting curves are shown in Figures 37a and 37b. As before, we see that the clamping levels are concentration dependent. However it seems that the clamping takes place much more sharply than the solution samples. The difference between the dropcoated and solution samples are shown in Figure 38. In this plot both samples are prepared using the $25 \%$ graphene in PVA solution. The solution is tested using a $2 \mathrm{~mm}$ cuvette. The dropcoated sample thickness is estimated to be around 10 microns. In Figure 38a we notice that the dropcoated sample exhibits a higher absorbance. This could be due to aggregation of graphene flakes on the substrate surface. However as seen in Figure 38b we see that the solid film shows higher output fluence when compared to the solution even though it shows a higher linear absorbance. The output fluence for the film could be greater due to its smaller path length - as the light interacts with the $2 \mathrm{~mm}$ cuvette over a longer length. However in Figure 38 (c) the solid film exhibits a lower input threshold. This leads us to believe that the other factors are affecting the optical limiting response in the solid. This could be due to 

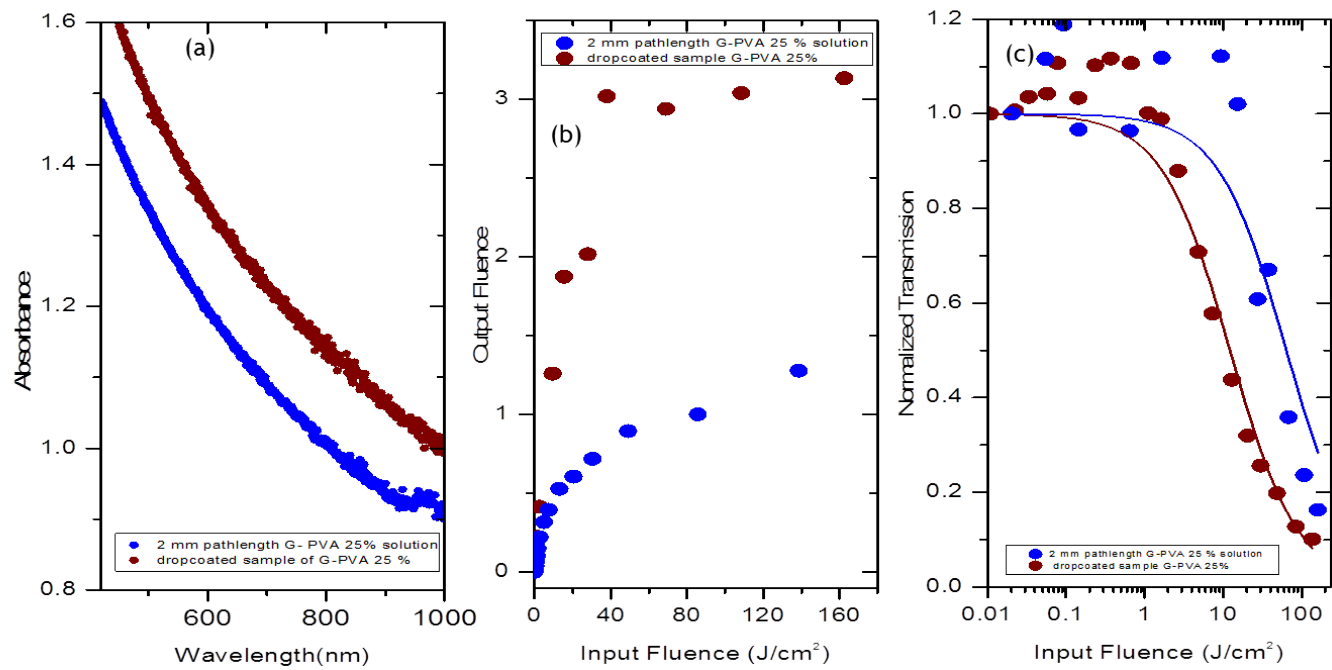

\section{Figure 38. Path length dependance of linear and nonlinear material transmittance (com- paring film to solution)}

(a) linear transmittance of various path lengths of graphene composite. (b) nonlinear output fluence of various path-length differences, and (c) normalized nonlinear transmission showing the influence of film-vs solution.

graphene flake aggregation, substrate and/or annealing dependent effects.

\subsubsection{Demonstration of Composite Structures}

From the pioneering work by Novoselov et al. ten years ago[45] significant volume of work has been devoted to investigations of electronic properties of graphene. Only recently has focus shifted on realizing the scope of graphene for optoelectronic devices. Much of this attention has been given to studying the nonlinear optical properties of graphene as applicable to the development of saturable absorbers (as described above) as well as optical limiting. Functionalized graphene-based suspensions have been used to display broadband optical limiting as defined in the seminal paper by Tutt and Boggess [47]. These optical limiting effects have been shown to occur due to mechanisms such as TPA, RSA and NLS. Although strong nonlinear behavior (in particular optical limiting behavior) in graphene materials has been demonstrated over a wide range of wavelengths the most promising results are mostly in suspension forms.[66-68]

This is due to the vulnerability of the solid host matrices to incur damage at high fluences. Further due to the high concentration of graphene in these suspensions optical limiting effects tend to dominate any saturable absorption due to a strong nonlinears scattering component, thereby not allowing broadband tunability of the nonlinear optical response in these samples. In this work we expand on these studies by developing low-cost, non-toxic graphene-based composites both in solution and spincoated form. Further we investigate the nonlinear optical properties of these composites for various pulse-widths (nanosecond to femtosecond) over a wide range of wavelengths. 


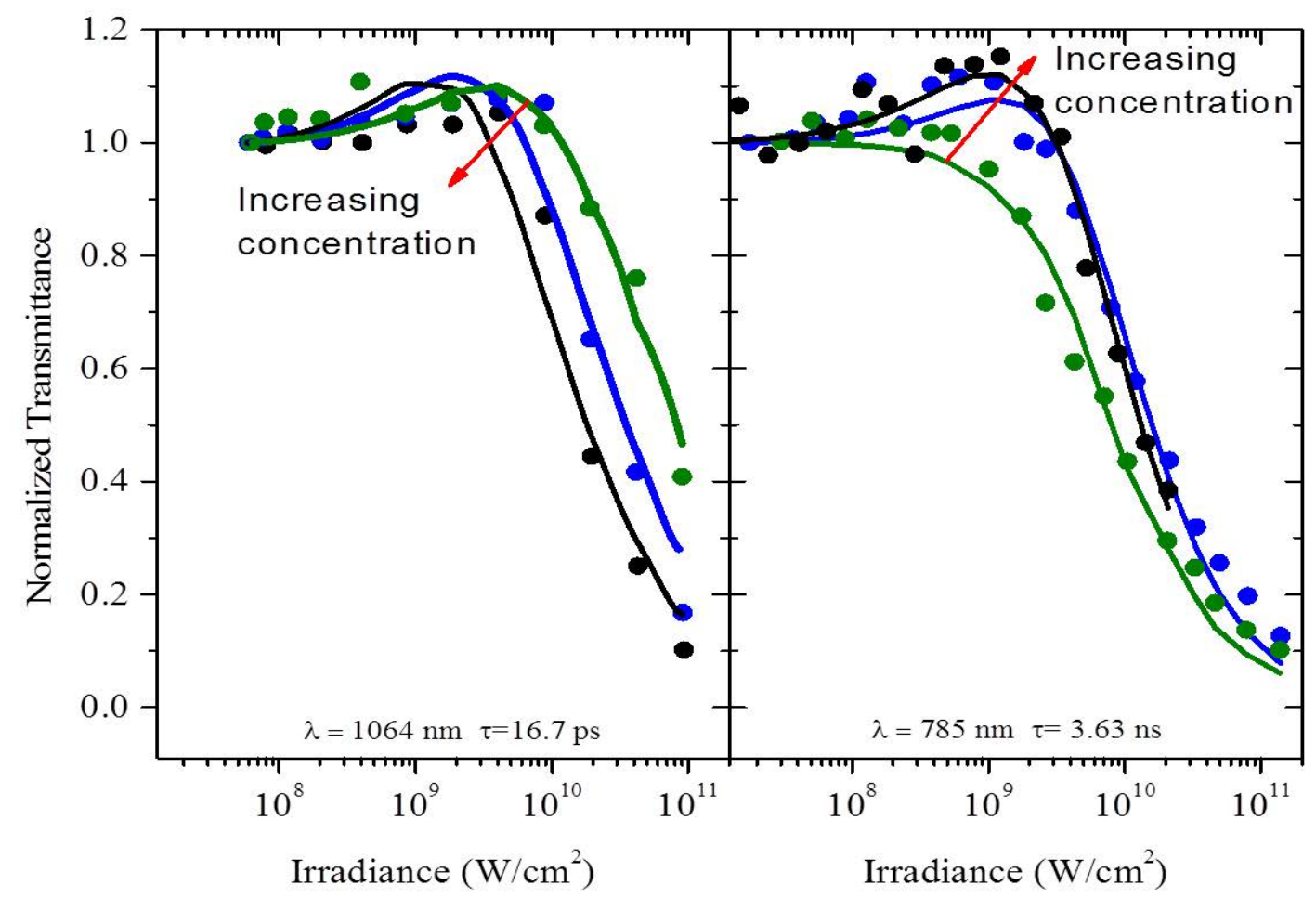

Figure 39. Demonstration of tunable nonlinear components with concentration

Shows the change in transmittance with irradiance for G-PVA solutions in the (a) ps (1064 nm) and (b) $\mathrm{ns}(785 \mathrm{~nm})$ regimes respectively. The onset of limiting for the G-PVA composites is lower in the ps regime (A) due to greater TPA contribution and lower saturable absorption. The greater saturable absorption observed for the high concentrations in the ns- (b) pulsewidth is due to greater ESA contribution. The nonlinear coefficients calculated via numerical fitting (solid lines) reveal high nonlinear absorption and lower saturation intensity for high concentration and longer pulsewidth. 


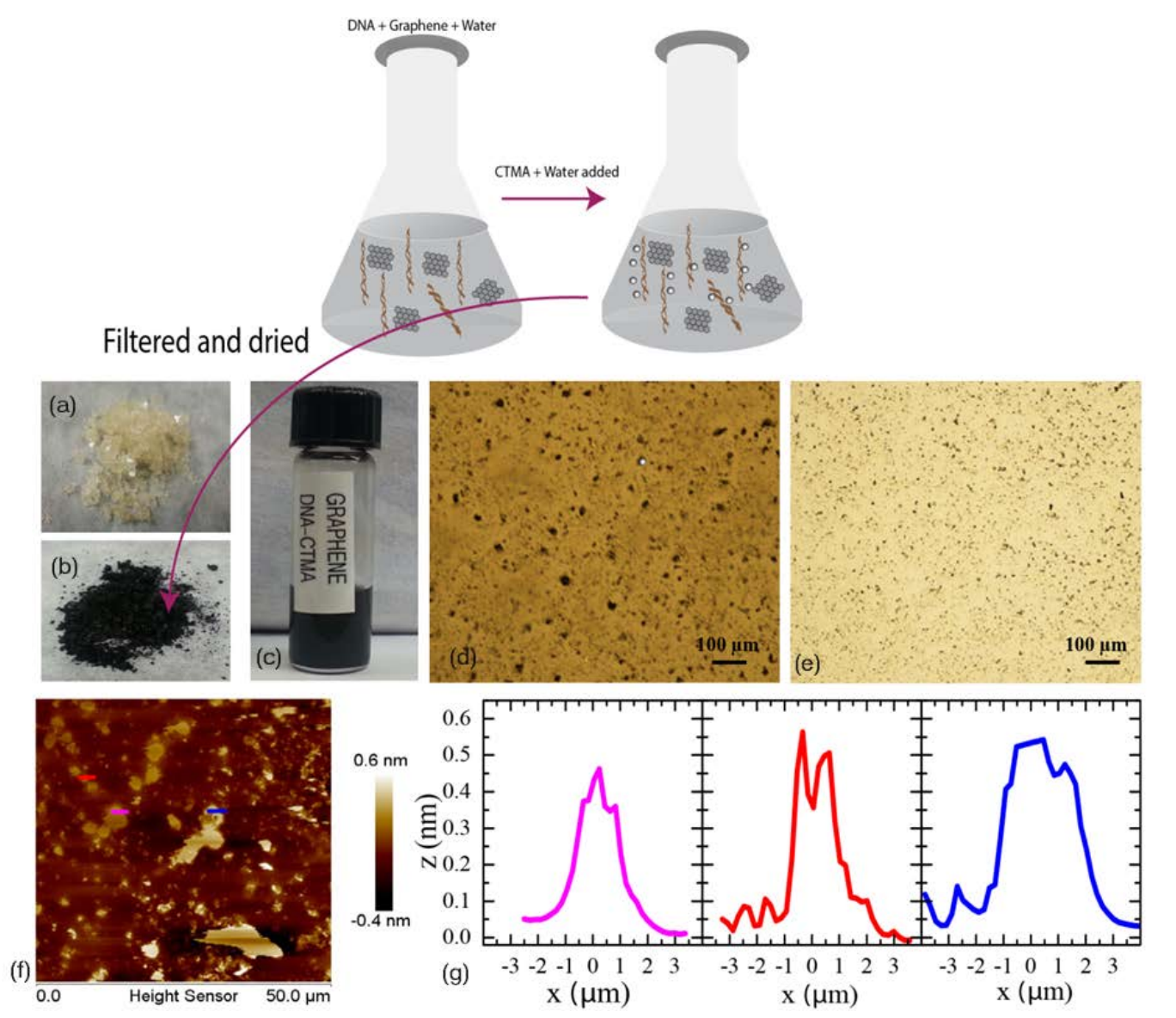

Figure 40. Steps for G-DNA composite processing

The off white DNA CTMA compound (a) turns to a gray compound (b) resulting in a G-DNA CTMA hybrid. This compound is dissolved in butanol (c) to form two concentrations (d) 25 $\mathrm{mg} / \mathrm{ml}$ and (e) $18 \mathrm{mg} / \mathrm{ml}$ and AFM measurements carried out show lateral sizes ranging from $1 \mu \mathrm{m}$ for non-aggregated flakes to $6 \mu \mathrm{m}$ for clustered graphene flakes

\subsubsection{Graphene DNA Composites}

Initially a graphene-polymer nanocomposite is developed by preparing a water-based graphene solution. Together with a surfactant this is dissolved in PVA composite in varying concentrations and sonicated. The resulting stable solutions are tested using an irradiance-scan (I-scan) technique in both the nanosecond (ns) and picosecond (ps) regime. Such a composite (in suspension form) indicated a very weak saturable absorption effect followed by strong optical limiting effects in the picosecond and nanosecond regime as shown in Figure 39 respectively. Nonlinear coefficients calculated indicated higher nonlinear absorption coefficient in the nanosecond regime as expected, due to strong scattering effects. Once spincoated however the PVA host was unable to provide thermal stability and damaged at fluences $<1 \mathrm{~J} / \mathrm{cm}^{2}$. Hence such a composite was deemed not suitable for device integration.

To try and alleviate these issues we attempted to integrated graphene into a DNA composite which has been shown to be a promising material for several optoelectronic devices as well as possessing high damage thresholds. The biopolymer used is formed from marine-derived DNA which has been explored as a bulk 

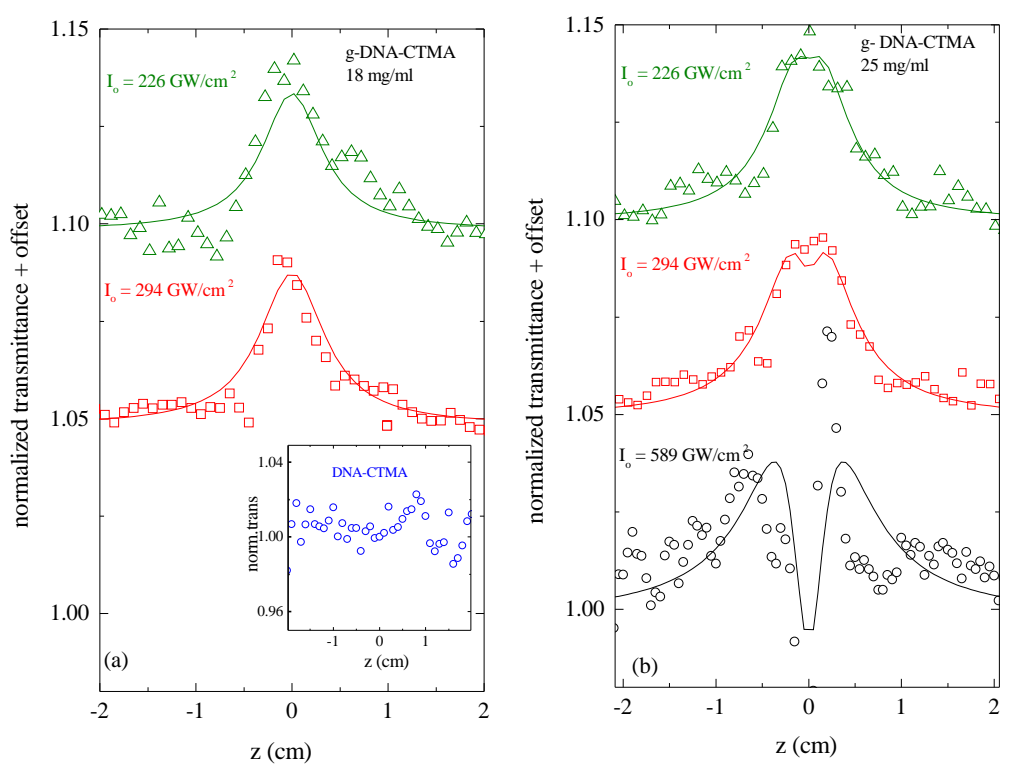

Figure 41. $z$-scan characterization of G-DNA films of various concentrations

(a) Peak intensity dependent z-scan measurements of $18 \mathrm{mg} / \mathrm{ml}$ shows saturable absorption in the form of a peak in transmittance not seen for the DNA-CTMA (inset) without graphene. With the concentration increased to $25 \mathrm{mg} / \mathrm{ml}$ shown in (b) higher pumping results in a dip in transmission whereby optical limiting effects start dominating in the sample in the form of TPA.

polymer material for photonic applications for the past decade.[69] Numerous devices and applications containing the DNA biopolymer have been demonstrated; these range from purely electronic applications such as field effect transistors (FETs) to optical applications such as light-emitting diodes (LEDs) and polymer electro-optic modulators.[70-72] The chemical structure of DNA allows for attaching particles, in this case graphene flakes, through either intercalation or major/minor groove binding, to the DNA double helix for enhanced material properties. These factors have therefore led this attempt to use DNA as a host matrix for graphene. This novel graphene-based bio-polymer results in combining the nonlinear behavior of graphene and the thermal stability and robust nature of a DNA composite. Graphene flakes (obtained commercially) are dispersed into a DNA-water solution, a surfactant (CTMA) is added and the graphene DNA-CTMA is precipitated, filtered and dried out to form a hybrid material (Figure 40 (b)).[69, 72-74] This newly obtained hybrid composite now allows one to dissolve it in any solvent. Here we use butanol to obtain uniform spincoated films. Material characterization of the composite itself is carried out and it is seen that the films are fairly uniform although some evidence of aggregation is noted in the AFM data shown in shown in Figure 40 (f) and (g)). $z$-scan measurements on the films with varying concentration (images of these film are shown in shown in Figure 40 (d) and (e)) are carried out at $\lambda=1 \mu \mathrm{m}$ in the femtosecond regime. The samples are irradiated with peak intensities ranging from $226 \mathrm{GW} / \mathrm{cm}^{2}$ to $589 \mathrm{GW} / \mathrm{cm}^{2}$. The z-scan measurements are fit using the same model described by Equation 32 .

As shown in Figure 41 (a) and (b), as the peak intensity is increased the saturable absorption effect in the lower concentration $18 \mathrm{mg} / \mathrm{ml}$ g-DNA-CTMA film increases giving us nonlinear coefficient of $0.5 \mathrm{~cm} / \mathrm{GW}$ and $I_{\text {sat }}=59 \mathrm{GW} / \mathrm{cm}^{2}$. Unlike lower concentrations, an increase in graphene results in the appearance of a dip in transmittance at comparable input intensities. For the $25 \mathrm{mg} / \mathrm{ml}$ concentration at $I_{\circ}=589 \mathrm{GW} / \mathrm{cm}^{2} 2$, optical limiting starts to overtake saturable absorption effects resulting in a higher NLA coefficient of $\beta_{\text {eff }}=$ 
$12 \mathrm{~cm} / \mathrm{GW}$ and subsequently higher saturation intensities. The inset in Figure 41 (a) shows no change in transmittance for the DNA-CTMA film demonstrating that the nonlinear response of the g-DNA-CTMA is indeed due to the embedded graphene. Outside the tunability of the nonlinear response, perhaps the most important feature of the graphene-DNA hybrid is how it responds to thermal effects and is able to potentially withstand high optical fluences without damage. Damage thresholds of these films are measured using a $3.63 \mathrm{~ns}$ pulse at a repetition rate of $1 \mathrm{~Hz}$. Initially no evidence of damage is seen below $178 \mathrm{~J} / \mathrm{cm}^{2}$ although humidity exposure does affect this threshold. This value is much higher than reported earlier for work done in testing damage thresholds in graphene materials. $[75,76]$ Currently more work is being done to improve this effect in addition to reducing aggregation of the graphene flakes. This will involve centrifuging the solution before spincoating to try and separate out the larger particles. In addition, circular dichroic measurements are also underway to study the preferred binding between the graphene flakes and the DNA helix.

\subsection{Graphene Saturable Absorber Mirrors}

Throughout this program, a key development effort has been focused on graphene as a saturable absorber in laser cavities to facilitate short-pulse operation. Graphene's extraordinary broad band nature provides an ideal platform upon which we may expand. The following introduces the design and characterization of the graphene and the saturable absorber mirrors (SAMs), as well as a way-forward in continued characterization of both graphene-based and semiconductor-based devices.

\subsubsection{Design of Saturable Absorber Mirrors}

When the graphene is located at a mirror and you have bidirectional propagation through the material, it is possible to utilize the standing wave to control the light-matter interaction with the graphene. As an example, the graphene exhibits a relatively flat linear absorption of $\sim 2.3 \%$. However, when integrated into a mirror, we can adjust the position of the extremely thin graphene relative to the standing mode. The resulting wavefunction overlap $(\xi)$ directly affects the linear loss. Figure 42 (a) shows how the field is controlled in the graphene layer. The confinement can be adjusted between nearly zero to 4 , where 1 is equivalent to unidirectional propagation through a transmissive graphene layer. At the center of the Bragg mirror, the confinement can be altered by adjusting the spacer layer. Figure 42 (b) shows how this is controlled for a given wavelength (in this case, $\lambda \sim 1 \mu \mathrm{m}$ ). While the spacer layer affects the reflected mode and therefore the standing wave slightly, the spacer layer thickness reaches maximum confinement when the antinode is located in the graphene, corresponding to a spacer layer thickness of $\lambda / 4$.

Figure 42 (c) shows the confinement factor of a graphene saturable absorber mirror (G-SAM) designed for $1.04 \mu \mathrm{m}$ again as function of spacer thickness, but for the full optical spectral band. However, it also presents the spectral dependance of the confinement. Figure 42 (b) represents a vertical scan at a wavelength of $1.04 \mu \mathrm{m}$. The optical spectral bandwidth of a G-SAM created using a spacer layer of $d$ thickness that would function over is much wider in the anti-resonant design (regions of blue) than in the resonant design (regions of red).

The reflectance of the G-SAM as a function of irradiance $(I)$ can be expressed as:

$$
\left.R(I)=\exp \left[-\xi\left(\frac{\alpha_{\circ}}{1+\xi I / I_{s a t}}\right)+\xi \beta_{\text {eff }} I\right) 2 L\right]
$$



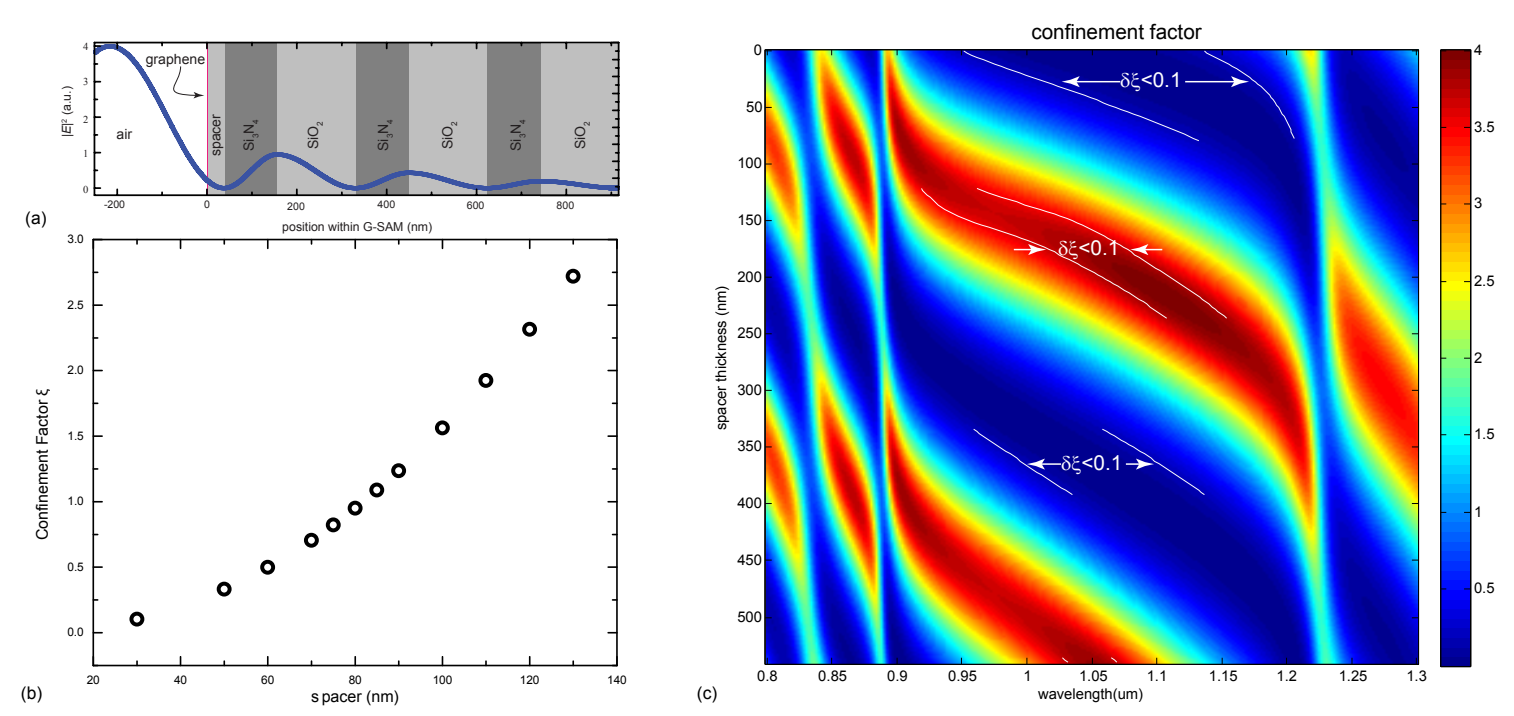

Figure 42. Calculated field-confinement varying spacer layer

(a) calculated field in a DBR structure with a spacer layer. Adjusting the spacer layer controls the field confinement $(\xi)$ within that layer, and therefore the light-matter interaction. (b) At a given wavelength, the confinement can be controlled through adjustment of the spacer layer thickness. (c) the spectral confinement of the field within the graphene as a function of spacer layer thickness. Anti-resonant structures (blue regions) have a much wider confinement width than the resonant structures.

where $I$ is the on-axis intensity, $\alpha_{\circ}$ is the linear absorption coefficient, $I_{s a t}$ is the saturation intensity, $\beta_{\text {eff }}$ is the nonlinear absorption coefficient, and $L$ is the actual thickness of the nonlinear material. The material saturable absorption can be expressed as a subset of this:

$$
\alpha(\xi I)=\frac{\alpha_{\circ}}{1+\xi I / I_{\text {sat }}}+\xi \beta_{\text {eff }} I
$$

The nonlinear reflectance of a G-SAM and dependance on the nonlinear components is shown in Figure 43 (a). The linear loss is controlled through the $\xi \alpha_{\circ}$ product. The modulation depth and unsaturated losses are controlled by $I_{\text {sat }}$ and $\beta_{\text {eff }}$ and their relation to each other. The device $I_{\text {sat }}$ is where the nonlinear losses are cut in half, and the roll-over in reflectance at high intensity is controlled by $\beta_{\text {eff }}$. We can control not only the linear reflectance through confinement, but the nonlinear terms $\left(I_{\text {sat }} / \xi\right.$ and $\left.\xi \beta_{\text {eff }} / \alpha_{\circ}\right)$ also adjust as a function of confinement. Figure 43 (b) shows the calculation of the nonlinear reflectance for three difference confinements. This amount of variation for a single absorber region is quite dramatic and reflects a great deal of customization and control possible through this method of graphene confinement.

\subsubsection{Characterization of Materials and Saturable Absorber Mirrors}

Prior to use in an antiresonant G-SAM structure, the graphene layer is characterized using a CARY ${ }^{\circledR}$ spectrophotometer, a Renishaw ${ }^{\circledR}$ Raman system and transmission mode $z$-scan. For material analysis, these measurements are performed with graphene on a quartz substrate, which does not interfere with the Raman signal or the nonlinear transmission measurements. The linear transmission for the graphene (with the quartz 

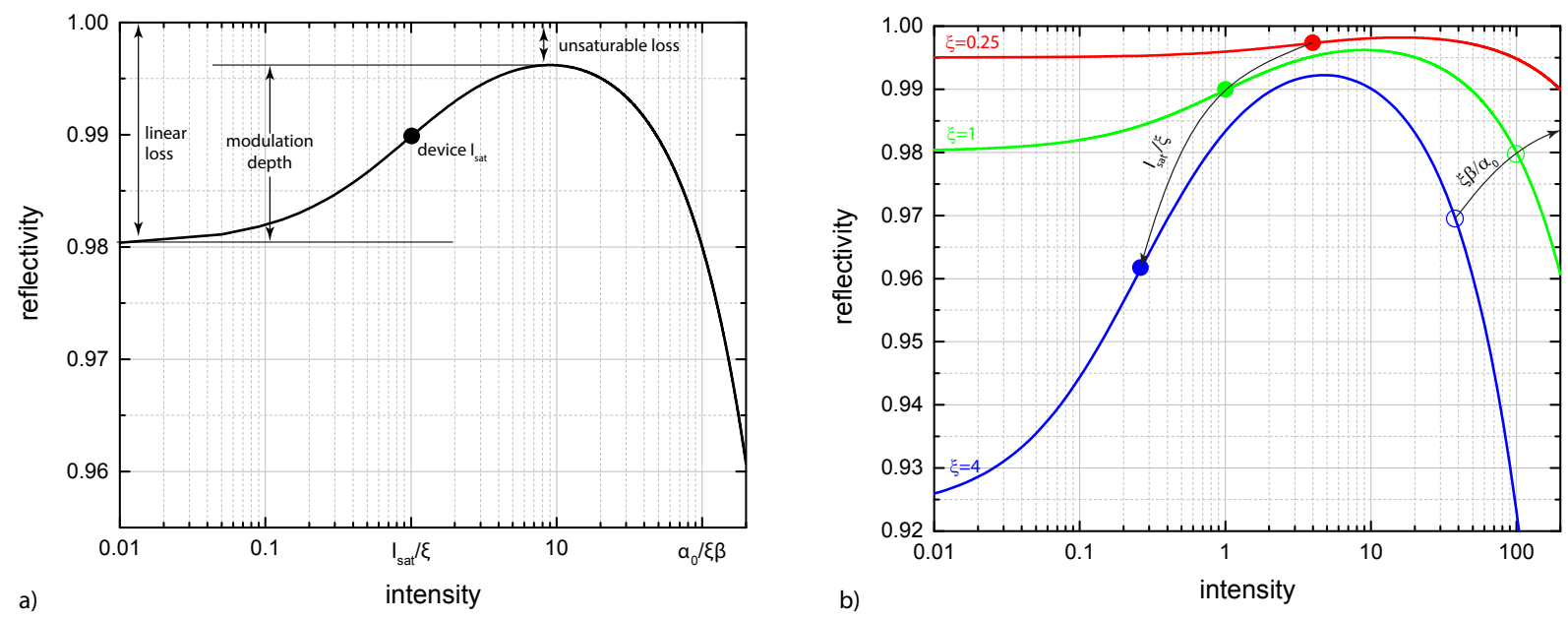

Figure 43. Calculated nonlinear reflectance for various GSAM designs

(a) Canonical intensity-dependent loss descriptions, including linear and nonlinear losses. b) Intensity-dependent nonlinear reflectance for single-layer graphene with spacer layers resulting in varied $\xi$.
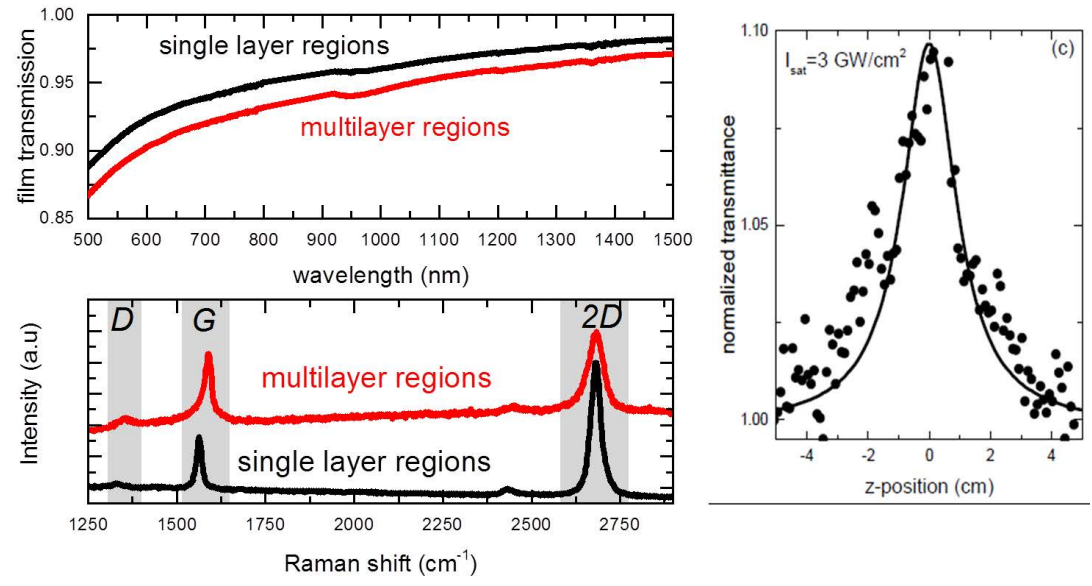

Figure 44. Linear and Raman graphene measurements from the visible through the nearinfrared region

(a) Linear measurements indicate a featureless linear transmission of graphene over a wide wavelength range (b) Raman measurements with a narrow, symmetric $2 D$ peak and $R_{2 D} / R_{G}>1$ indicates monolayer graphene although some areas exhibit Raman spectra characteristic of multilayer regions (c) z-scan measurements of the single film with a peak in transmittance indicates saturable absorption with $I_{\text {sat }}$ of $3 \mathrm{GW} / \mathrm{cm}^{2}$. 
background removed) is shown in Figure 44 (a). Here we notice a flat transmission spectra over a wide wavelength range indicating the broadband nature of graphene with some variability depending on location, presumably due to regions of multilayer graphene.

The films are then studied using Raman measurements at $514 \mathrm{~nm}$, with a $50 \times$ objective and a 1800 1/mm grating. The Raman spectra of one such transferred film is shown at two different points in Figure 44 (b). As expected the Raman spectra of carbon-based materials exhibit three prominent peaks. The $D$ peak located at $\sim 1329 \mathrm{~cm}^{-1}$ is activated in the presence of defects. The $G$ peak (at $1562 \mathrm{~cm}^{-1}$ ) corresponds to the doubly degenerate phonon mode at the Brillouin zone center while the $2 D$ peak at $2684 \mathrm{~cm}^{-1}$ is a two-phonon band which exists in the second order Raman spectra of graphene irrespective of defects.[77, 78] Here we observe that the $D$ peak is suppressed indicating a nearly defect free graphene surface. The small $D$ peak that is observed is likely due to wrinkles or folding in the graphene transferred from the copper. The $2 D$ peak is symmetric, undistorted and can be fit to a single Lorentzian with a full width half maximum (FWHM) of $33 \mathrm{~cm}^{-1}$. This, together with the intensity ratio $R_{2 D} / R_{G}>1$, indicates monolayer graphene. It should be mentioned that although the response over a large part of the surface indicated monolayer graphene, certain areas do exhibit a lower, symmetric $2 D$ peak indicative of multilayer regions. [77-79]

A sub-picosecond $z$-scan at wavelengths ranging from $1020 \mathrm{~nm}-1086 \mathrm{~nm}$ is used as a means to probe the graphene layer saturable absorption. The $z$-scan setup consists of a tunable Ti:sapphire laser source with a $125 \mathrm{fs}$ pulse width and a $1 \mathrm{kHz}$ repetition rate. For the data presented in this summary, the pulse energy was $0.77 \mu \mathrm{J}$ at a wavelength of $1086 \mathrm{~nm}$. The beam has a Gaussian profile with a beam waist radius of approximately $25 \mu \mathrm{m}$. The Rayleigh length $\left(z_{R}\right)$ is calculated to be $0.25 \mathrm{~cm}$. Measurements are taken in transmission, where the peak fluence is given by $I_{\circ} /\left(1+z^{2} / z_{R}^{2}\right)$. The $z$-scan measurement is shown in Figure 44 (c) whereby the peak in transmission indicates saturable absorption, as the sample is translated through the focal point. The transmission varies slightly over the surface of the sample due to regions of multilayer graphene. To quantify this contribution, data is fit to an intensity-dependent absorption model consisting of both saturable absorption and optical limiting terms of the form of Equation 32.[80-82]

Based on the relatively low fluences tested, it is sufficient to neglect $\beta_{\text {eff }}$, although if a laser is operated at larger fluence, effects such as two-photon absorption must be considered. The fit shown in Figure 44 (c) assumes three layers of graphene and results in a saturation intensity of $3 \mathrm{GW} / \mathrm{cm}^{2}$ which is comparable to previous reports. $[6,83]$ At other points, $z$-scan measurements indicate single layer graphene, although this data has larger relative noise. Additional measurements are carried out at lower fluences and various wavelengths for thorough analysis. Although the $I_{\text {sat }}$ does not change dramatically, the signal-to-noise ratio over the sample does vary with decreased input fluences.

Based on this material characterization, we calculate the optimum $\mathrm{SiO}_{2} / \mathrm{Si}_{3} \mathrm{~N}_{4}$ antiresonant structure demonstrated here utilizes a spacer layer $(37 \mathrm{~nm})$ designed to give a low field enhancement at the graphene interface. Here the field enhancement at the graphene surface is approximately 0.25 which leads to a G-SAM saturation intensity $\left(I_{\text {sat }}^{(e f f)}\right)$ of $12 \mathrm{GW} / \mathrm{cm}^{2}\left(I_{\text {sat }}^{(e f f)} \equiv I_{\text {sat }} / \xi=3 / 0.25 \mathrm{GW} / \mathrm{cm}^{2}\right)$. [79] This low confinement is beneficial for producing high pulse energies and damage thresholds, but can be tailored based on the application and needed response of the saturable absorber mirror. As shown in Figure 42 (c), this also corresponds to a very broad nonlinear reflectance band, which can be utilized to mode-lock a broad spectral range of lasers, and has been used to do so in Reference 6.

To better understand the VECSEL effects described above, we are developing an in-house nonlinear reflectance measurement system to probe both the VECSEL chip gain and nonlinear optical characterization 


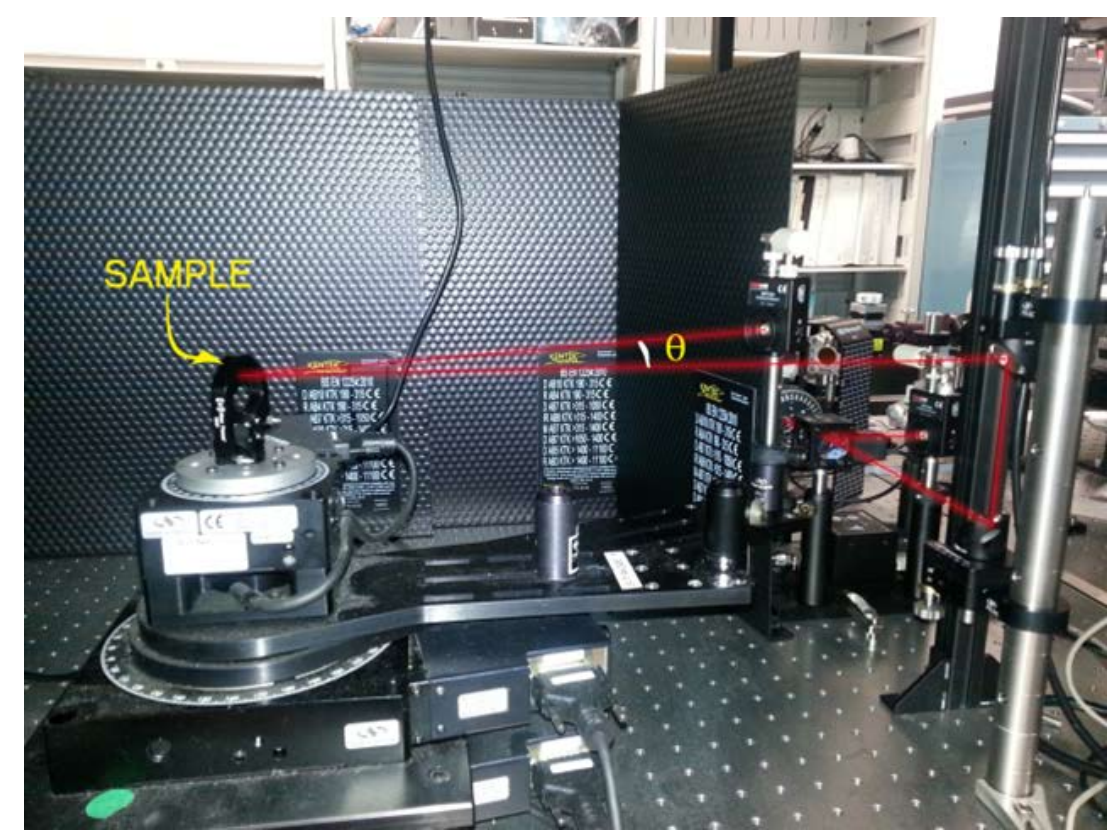

\section{Figure 45. Nonlinear reflection measurement system}

angle dependent nonlinear reflection setup currently underway using a femtosecond high power laser.

of the G-SAM. Currently our setup consists of an angle dependent reflectivity setup using a goniometer and high speed detectors (Setup shown in Figure 45). The reflectivity of the SAM will be calculated as a function of incident pulse fluence which is changed using a variable attenuator consisting of a combination of a half-wave plate and polarizer. Beam profiling was carried out to determine the fluences which will be needed to saturate the graphene. The pulse fluence on the graphene SAM should be at least $10 \times$ the saturation fluence. Such a measurement will allow us to study and characterize each saturable absorber mirror (with and without the graphene) to determine parameters such as saturation fluence and modulation depth. These measurements are extremely useful in predicting the most optimum design to achieve high power, stable mode-locking. Once the parameters needed to saturate the graphene are accurately calculated using the nonlinear reflection setup similar intracavity fluences will need to be induced in the VECSEL cavity setup.

\subsubsection{Growth and Fabrication}

We first introduce the gain component of the cavity that we use to experimentally explore VECSEL mode-locking. The chip is composed of strain compensated InGaAs quantum wells and GaPAs barriers, followed by an AlAs/GaAs Bragg mirror grown using metal-organic vapor phase epitaxy (MOVPE) by Prof. Wolfgang Stolz of University of Marburg. The structure is bonded epitaxial-side down to a diamond heat-spreader and the substrate is completely removed, with an anti-reflection coating applied to the surface to maximize the pump in-coupling. The semiconductor structure is optimized for high power operation at nominally $1030 \mathrm{~nm}^{2}$

\footnotetext{
${ }^{2}$ More information can be found in a similar structure - Heinen, et al., Electron. Lett. 48, 516 (2012) and is provided by Prof. Jerome Moloney of U. of Ariz.[84]
} 


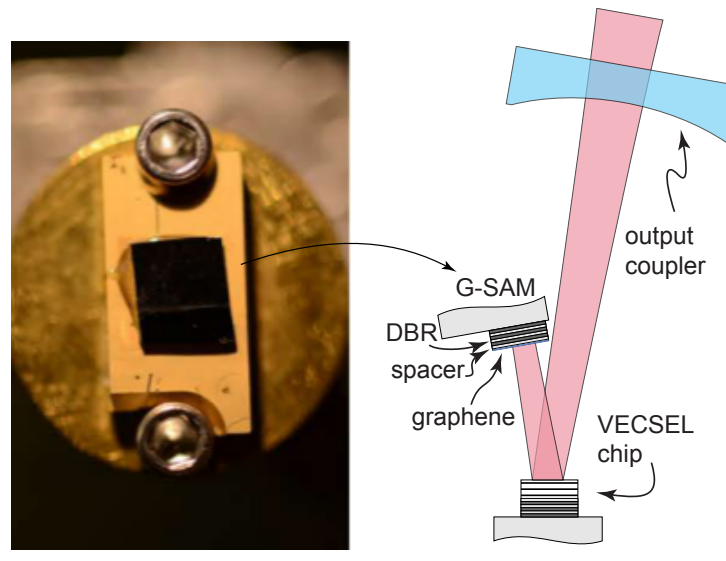

a) b)

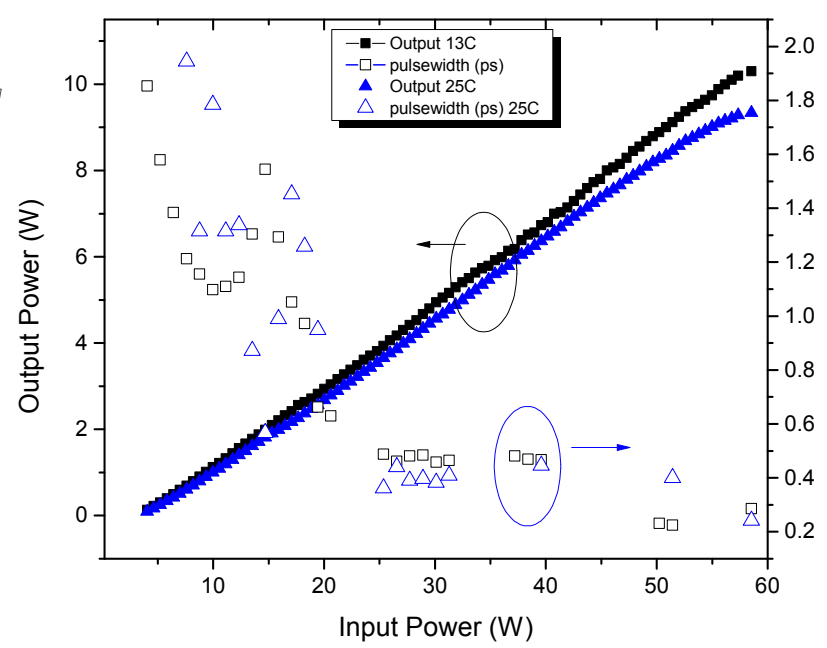

c)

\section{Figure 46. V-cavity schematic and power characteristics}

(a) Micrograph of G-SAM epoxy-mounted to a copper die and mounted in custom cavity mount.

(b) Shows a schematic of the V-cavity with the gain chip at the fold and the G-SAM and outcoupler at either end. Such a cavity with length $8.5 \mathrm{~cm}$ results in (c) power curve for the VECSEL cavity whereby we were able to attain an average power of $10 \mathrm{~W}$ with sub-picosecond pulse widths, also shown as a function of pump power.

The G-SAM integrated in the VECSEL cavity consists of the DBR which possesses 9 periods of alternating layers of chemical vapor deposition (CVD) silicon dioxide $(177 \mathrm{~nm})$ and silicon nitride $(119 \mathrm{~nm})$ with a $37 \mathrm{~nm}$ silicon dioxide spacer. The graphene is transferred from a commercially purchased graphene on copper foil. The graphene-Cu is spincoated with PMMA at $4000 \mathrm{rpm}$ for 40 seconds. The foil is then immersed in a $1 \mathrm{M}$ ferric chloride solution in water until the copper is etched. The free-standing PMMA-graphene film is then rinsed with water and methanol and lifted off using the DBR. The sample is then heated for 20 minutes at $100^{\circ} \mathrm{C}$ to flatten out the film. The PMMA is finally removed using acetone. $[85,86]$

\subsubsection{Graphene Mode-locked Laser Characterization}

We use a standard V-shaped cavity to obtain mode-locking. The G-SAM is placed at one end while a high reflectivity (98\%) outcoupler with a $10 \mathrm{~cm}$ focal length is placed at the other, folded about the VECSEL chip. A schematic of the cavity is shown in Figure 46 (a). The VECSEL chip is mounted on a heat sink maintained at $13^{\circ} \mathrm{C}$ for temperature control, and is pumped using a fiber coupled laser diode operating at $808 \mathrm{~nm}$. The total length of the cavity is $8.5 \mathrm{~cm}$ with a $127 \mu \mathrm{m}$ spot size on the G-SAM which leads to a maximum intensity of $2.5 \mathrm{GW} / \mathrm{cm}^{2}$ at the G-SAM. This intensity does not lead to any damage of the graphene although damage thresholds lower than this have been reported. [87] The high damage threshold here we believe is the result of the low field confinement at the graphene layer. The cavity exhibits large mode-locked output powers, shown in Figure 46 (b). Here we report the dependence of output power and pulsewidth as a function of the pump power, achieving 10.2 W out of a VECSEL cavity without any significant signs of roll-over. An increase in input power also results in a decrease in the pulsewidth of the system since the higher pumping 
results in greater saturation of the graphene.

The optical spectrum bandwidth also changes with input pump power, maintaining nearly bandwidth limited pulses throughout the entire region measured. At powers below about $6 \mathrm{~W}$, the optical spectrum is primarily single lobed, with a single-peak autocorrelation function as shown in Figure 47 (a) and (b) for an output power of $2 \mathrm{~W}$. Here the autocorrelation spectra and calculated function consists of a single symmetrical peak fitted to give a pulsewidth of 500 fs which decreases with higher pumping. The optical spectrum and autocorrelator spectra for this cavity are presented at higher output power of $9.3 \mathrm{~W}$ in Figure 47 (c). The emission spectra as shown in 47 (c) displays an asymmetric optical spectra with a FWHM of $6.92 \mathrm{~nm}$ centered at $\sim 1044 \mathrm{~nm}$. The corresponding three-peak autocorrelation function pulse is shown in Figure 47 (d). While the autocorrelation function does not provide a unique pulse shape, one possible temporal pulse envelope consistent with such a function contains two, $350 \mathrm{fs}$ FWHM sech ${ }^{2}$ pulses separated by approximately $650 \mathrm{fs}$. Although this may not be a unique solution, the spectral phase of such a "test" pulse is given in Figure 47 (c). The center autocorrelation peak is fit with a $\operatorname{sech}^{2}$ function to show agreement with a pulse width of $353 \mathrm{fs}$. The function obtained via the autocorrelated discrete Fourier transform of the optical spectra, including test phase from Figure 47 (c), is shown in the shaded region in Figure 47 (d), which is very close to transform limited, and is in good agreement with the experimental autocorrelation signal. Finally evidence of mode-locking was verified via radio-frequency $(\mathrm{RF})$ spectrum measurements presented in Figure 47 (e) with a signal-to-noise of $50 \mathrm{~dB}$ at $1.76 \mathrm{GHz}$ [ $<30 \mathrm{~dB}$ in Figure 47 (e)] from higher noise floor resulting from larger bandwidth resolution), and strong harmonics up to our resolved limit of $26.5 \mathrm{GHz}$, limited both by our detector and RF spectrum analyzer. The RF harmonics vary more than expected, and the implications of these results remain unclear. However, this RF spectrum is generally consistent with two simultaneous pulses traversing the cavity. If this holds true, these sub-picosecond pulse envelopes each have energies of approximately $2.8 \mathrm{~nJ}$. This large average power compares favorably to semiconductor saturable absorber mirror (SESAM) mode-locked VECSELs.

The G-SAM mode-locked VECSEL has a maximum operating internal cavity peak power of approximately $2.5 \mathrm{GW} / \mathrm{cm}^{2}$, while the effective $I_{\text {sat }}$ is closer to $12 \mathrm{GW} / \mathrm{cm}^{2}$, once the optical field overlap is considered. Mode-locking is occurring below the saturation fluence, in the same operation mode previously seen in SESAM mode-locked, as well as graphene mode-locked lasers.[87]

In an effort to experimentally demonstrate the influence of the field confinement, we developed a G-SAM consisting of the silicon dioxide/silicon nitride mirror and a $86 \mathrm{~nm} \mathrm{SiO}$ spacer with a confinement of near unity at the graphene layer. In addition, to increase the fluence at the graphene layer we increased the length of our cavity and decreased the spot size on our G-SAM because the losses (both linear and nonlinear) become larger and the effective $I_{\text {sat }}$ increases (indicated in Figure 43 (b)). Although powers as high as before were not achieved we have still measured higher average power than previously published and were able to get a more stable and narrower linewidth RF spectrum shown in Figure 48 (a) and single lobed optical spectrum shown in Figure 48 (b). Once we determine the nonlinear coefficients and optimize the geometric parameters of the various G-SAMs developed, we intend to place them in this VECSEL and demonstrate more stable mode-locking at high powers. 

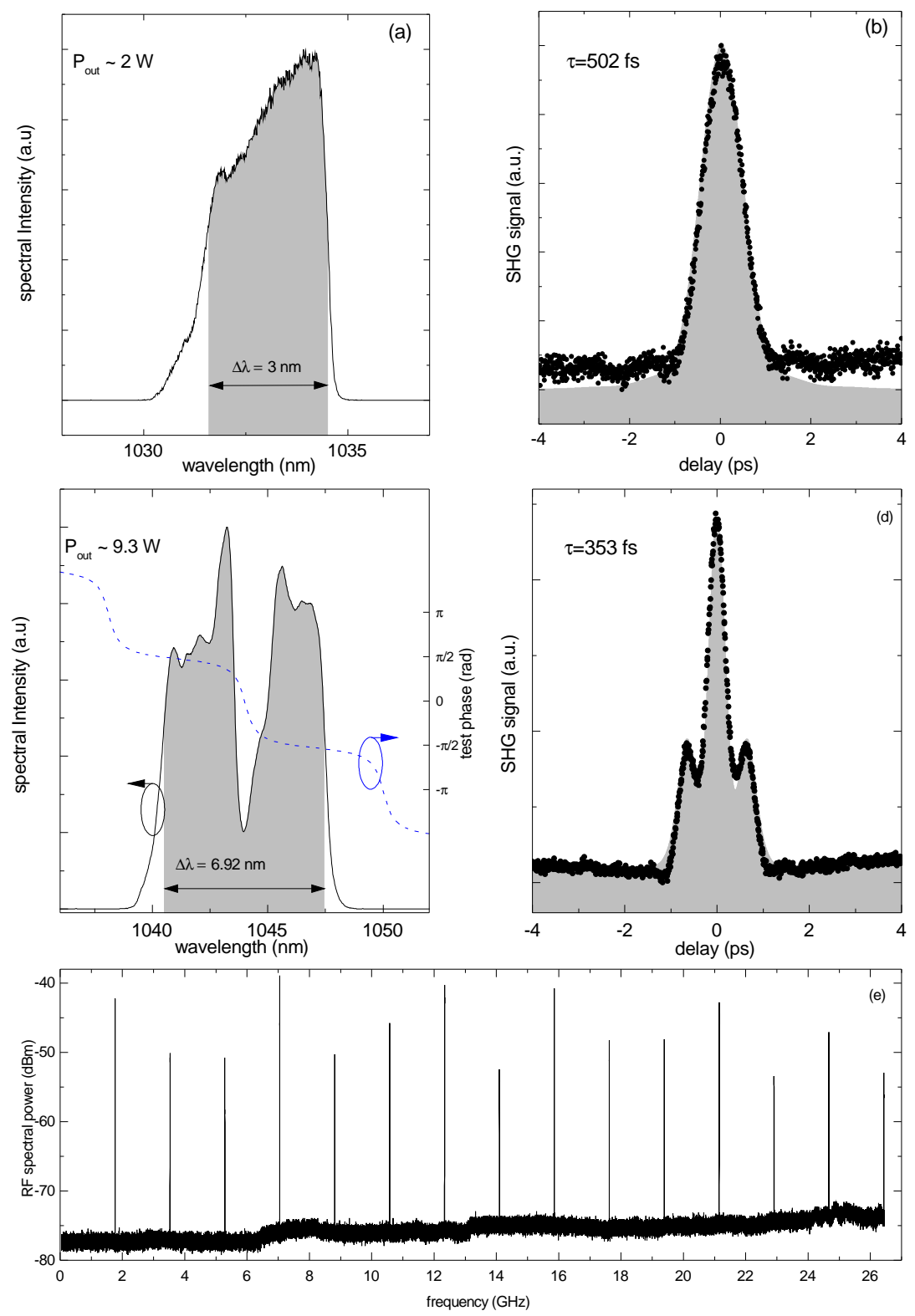

\section{Figure 47. Optical spectrum, autocorrelation, and RF spectrum}

(a) Shows the optical spectrum of the VECSEL cavity operating at a low power of $2 \mathrm{~W}$ with a spectral bandwidth of $3 \mathrm{~nm}$ corresponding to single peak (b) autocorrelation trace (dots). At higher powers this optical spectrum becomes asymmetric as shown in (c) with the optical spectrum of the VECSEL cavity operating at $9.3 \mathrm{~W}$, with a spectral bandwidth of $6.92 \mathrm{~nm}$ corresponding to a nearly Fourier transform limited pulse which corresponds to $(d)$ the autocorrelation trace (dots) with side lobes. The FWHM of this side-lobed autocorrelation has a resulting optical pulse of $353 \mathrm{fs}$ (resulting from sech ${ }^{2}$ fit to central peak). The shaded region indicates the calculated autocorrelation trace from the corresponding optical spectrum using the test phase given in (c). (e) RF spectrum showing the first 15 harmonics. 

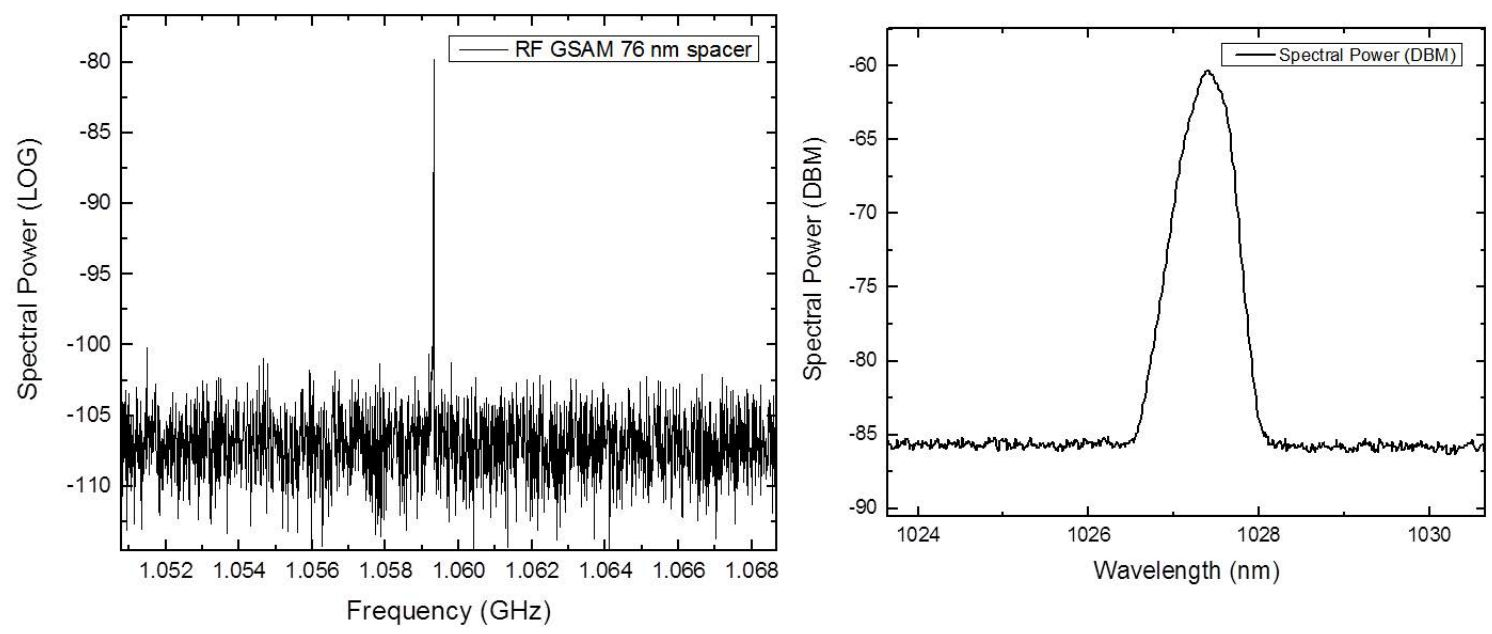

Figure 48. Narrowed RF spectrum indicating stable mode-locking

(a) Narrower $R F$ spectra of the first harmonic $(<3 \mathrm{kHz})$ and the single lobed optical spectrum in (b) indicates potential stable mode-locking in the system with the higher confinement G-SAM 


\subsection{ASSOCIATED RESEARCH}

During this period, there was some research that resulted as part of this project, but the output was not directly related to the end-product of the effort. They are worth recording within this report.

\subsection{Sb-Nanowires}

While growing III-Sb structures, we encountered a troubling result - that is there were surface-defects after finalizing the epitaxial growth, especially for thick structures which are typically needed for longer wavelength devices. As an example, this occurred on thick antimony-based structures such as DBRs requires an open antimony shutter to remain open for the entire duration of the growth. Excess antimony condenses on several cold surfaces during this process, including the cracked source shutter. At the conclusion of the antimomide device growth, the substrate is cooled under an antimony overpressure until the temperature of the wafer reaches $\sim 400^{\circ} \mathrm{C}$, a process which prevents surface deterioration resulting from antimony desorption. At this point, the antimony shutter is closed and the substrate is cooled down to ambient chamber temperature. For long antimonide runs however, the accumulation of antimony on the back of the antimony shutter heats up during the cooling process, due to its close proximity of the antimony cracking. As the backside of the shutter is vaporizing trace amounts of antimony, the substrate is already near ambient temperature. The antimony hence crystalizes on the cold wafer surface resulting in nano-crystal formation. We have thus generated DBR samples that have surface antimony and some that are free of surface antimony, achieved by using the cracker valve to remove the Sb overpressure. The primary reason for expecting the presence of antimony on the DBR surface is the significant change in the reflectivity of the samples for conditions with and without antimony.

We used a series of analysis to determine the result of this surface structure. First, atomic force microscopy was used to notice there were surface structures similar to nanowires, all aligned with each other and orientated in nominally the same direction. An example of this can be seen in Figure 49a. To determine the composition of these features, cross-section transmission electronic microscopy (X-TEM) is used to investigate the surface of the DBRs, and is imaged in Figure 49b, which shows three distinct regions of the nanowires, each which may be analyzed using cross-sectional EDS. The first region (i) is the underlying substrate, which has signal indicating it is pure AlSb (in the example shown in Figure 49 the nanowire was grown on AlSb). Region (ii) is an interfacial region where the aluminum is quenched and replaced by $\mathrm{Sb}$ to form $\mathrm{GaSb}$, as indicated by Figure 49c. After this wetting layer, the nanowires form through strain-induced growth, which causes pyramidal structures of pure Sb. While it can not be seen in Figure 49b, each pyramid is composed of two domains growing normal to the pyramid surfaces.

Polarization dependent transmission measurements are then carried out using a UV-VIS-NIR Cary spectrophotometer. Here the measurement is taken in a standard manner with polarizers placed in the path of both the sample and the reference beams. Transmission measurements were taken with both the orthogonal incident polarization directions (which we refer to as $T_{v}$ and $T_{h}$, polarized in the $v$ and $h$ directions, respectively). As expected, it was seen that transmission measurements taken at normal incidence on the DBR without the antimony deposits (not shown) show no difference between the $v$ and $h$ polarization directions. However, polarization selectivity is seen for the DBR consisting of the antimony nano-structures

whereby the transmission intensity of the sample is higher when incident with $v$ polarized light (wherein $\vec{E}$ 
a)

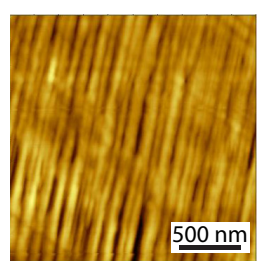

b)
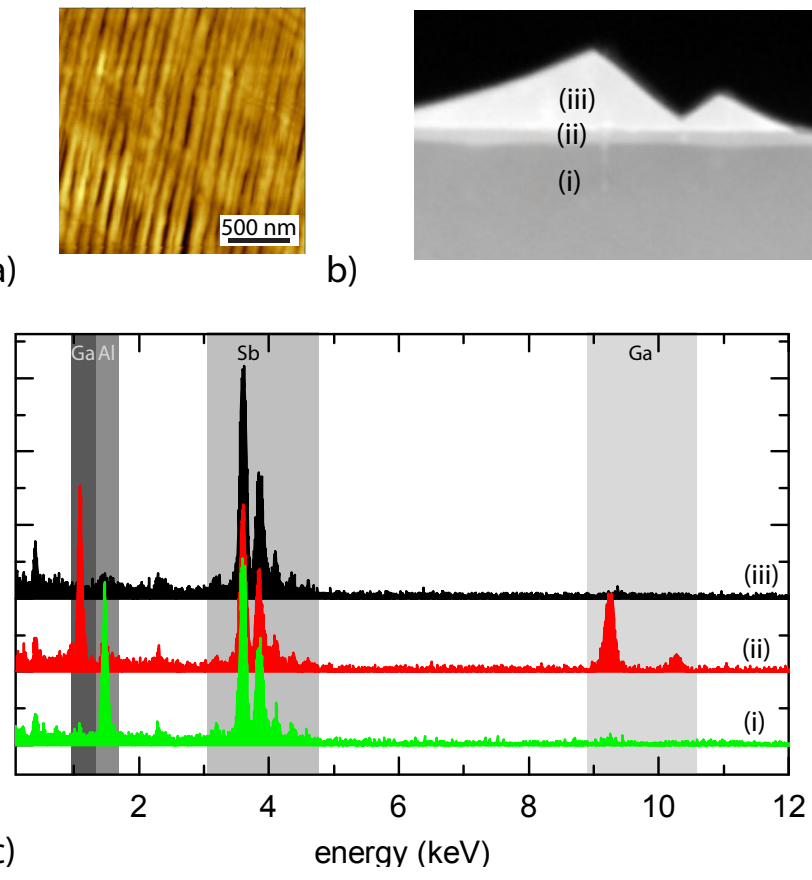

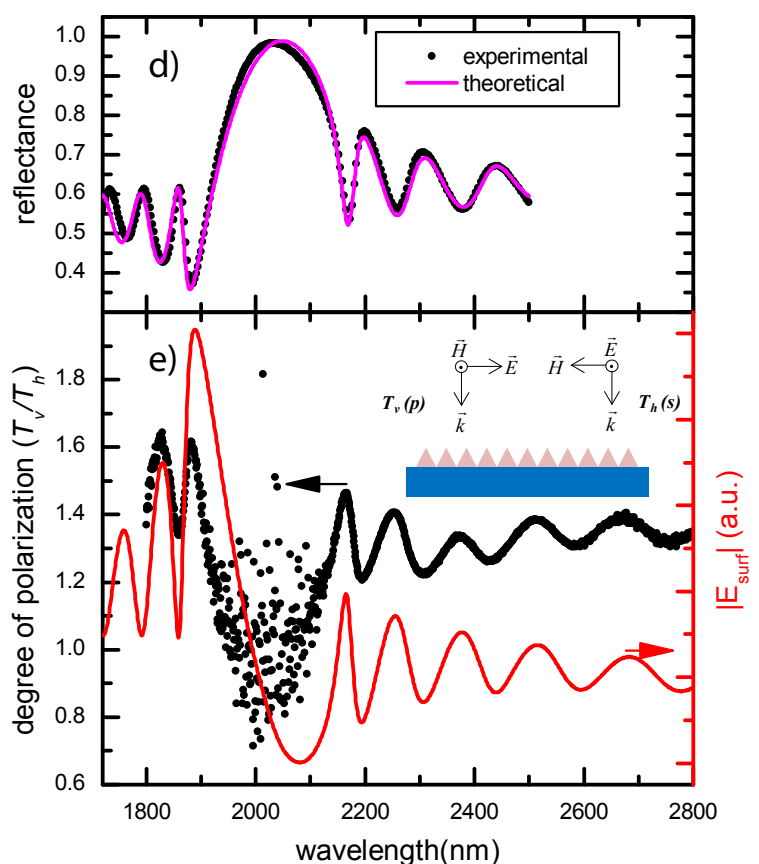

Figure 49. Analysis of Sb-nanowires on GaSb

a) surface atomic force microscopy, b) XTEM of surface structure, c) cross-sectional analysis EDS of layers within the structure, . d)Transfer matrix-based simulations are used to fit the modified reflectance of antimony-based DBR by using a calculated antimony-air composite refractive index. e) Degree of polarization shows a higher transmission for vertically-polarized light. In addition, the amplitude of the electric field at the Sb nano-structure surface coincides with the enhancement of polarization. A cross-section schematic of the antimony-nano-structure surface is inset indicating the direction of $v$ and $h$ polarizations. 
is perpendicular to the $\mathrm{Sb}$ nanowires on the surface). A schematic of the cross-section of the antimony surface and the polarization directions relative to the antimony nanowires is shown in the inset of Figure 49e. The degree of polarization $\left(T_{v} / T_{h}\right)$ as well as the electric-field profile at the surface of the nano-structures is shown in Figure 49e. Here it can be seen that the DBR with the antimony nanowires primarily favors transmission of vertically-polarized light. The electric field profile is calculated using the antimony-air index and thicknesses of the layers. The field intensity coincides with the degree of polarization indicating that the optical field is more confined within the Sb nano-structure layer, this allows for greater light-matter interaction thereby enhancing the effects of the nano-structures. The DBR below allows for the tuning of these resonances by changing layer thickness, indices as well as the incident angle. This indicates that the antimony nanowires behave much like a wire-grid polarizer whereby light polarized perpendicularly to the antimony nanowires has a higher transmission. This discriminating of light polarization however is not significant due to the possible coupling between the nanowires due to their extremely close proximity.

\subsection{Laser Packaging}

Indium is a key metal for making electrical as well as thermal contact to a wide variety of semiconductors. Despite some disadvantages such as whisker formation and migration under lifetime test[88], key characteristics such as its mechanical compliance and softness, combined with extremely high thermal- and electrical-conductivity make it a preferred contact metal for applications where the solder is sandwiched with some force between a device and carrier. For instance indium solder technology is critical for bonding detector arrays to readout integrated circuits (ROIC) to form focal plane arrays (FPAs)[89] as well as some high power devices such as power amplifiers or large area lasers with heat spreaders[90]. In order to achieve such a layer of indium, several different techniques have been employed including - electroplating[91], vacuum deposition by sputtering[92] and evaporation[93]. The deposition techniques have offered better control over the thickness of the indium film and are currently employed extensively in the semiconductor processing industry. Several significant challenges posed by deposited indium on various substrates include void formation which can be highly detrimental to thermal transport[94], as well as the presence of extensive crystalline columns on the film surface which can complicate bonding to the indium surface. It must also be pointed out that the softness of indium which makes it such a desirable solder prevents the use of any mechanical or chemical polishing techniques from improving the surface.

To overcome these indium thin film voids and improve surfaces more amenable to uniform bonding, we have explored DC sputtering of indium onto silicon substrates with using the substrate temperature as a key parameter in achieving closely packed and highly planar films. The planar surface morphology of a sputtered metal is highly dependent upon the homologous temperature at which the process takes place. The homologous temperature of the sample is defined as the ratio of the substrate temperature during sputtering to the melting point of the metal. It has been shown by Grovenor et al.[95] and by Thornton[96] in their structure zone models that the deposition of sputtered metal films at homologous temperatures of $<0.2$ results in very small equiaxial grains and a planar surface morphology whereas at a much higher homologous temperature of $>0.7$ the growth would be dominated by very large columnar structures. The presence of these large columnar structures is considered to be the dominant source of surface roughness when sputtered indium is used as a solder[97]. In most metals the melting point is very high compared to ambient room temperature, and therefore the grain structure corresponding to zone I or zone $\mathrm{T}$ is naturally produced with a room-temperature platen as the homologous temperature remains $<0.2$. In the case of indium, the low melting point of $429.76 \mathrm{~K}$ results in a substrate temperature of $\sim 86 \mathrm{~K}$ necessary to achieve a homologous 


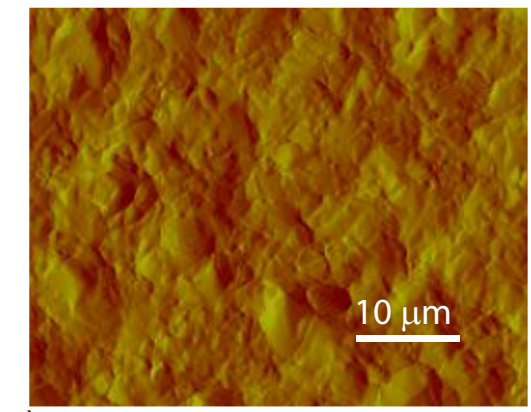

a)

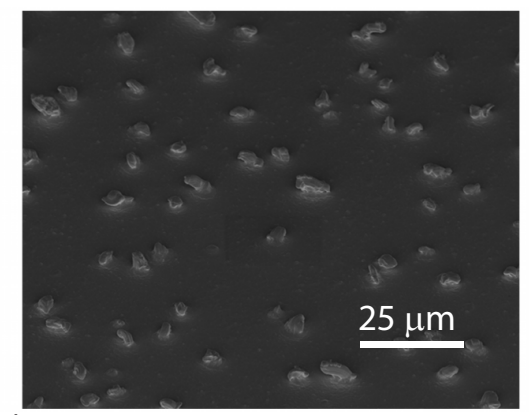

b)

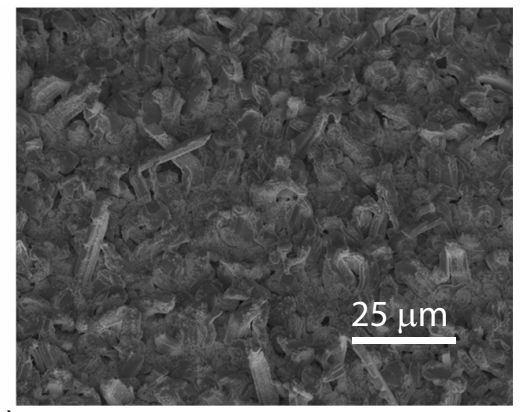

C)

Figure 50. AFM and Crossectional SEM of indium deposited above and below homologous temperature

SEM image of the samples with indium sputtered on a silicon samples at $77 \mathrm{~K}$ substrate temperature and at room temperature. The cryogenic sample in part (b) clearly shows significant reduction in the presence of crystalline indium grains compared to the room temperature sample in part (c). Part (a) shows the AFM profile of the planar region in the cryogenic sample.

temperature of 0.2 and this can be achieved by cooling the substrate with liquid nitrogen during indium sputtering. This study involves comparing two scenarios. The first scenario represents indium deposition with the substrate kept at room temperature (no active temperature control), where the homologous temperature is $\sim 0.70$. The second scenario involves depositing indium with a homologous temperature of about 0.17 and is achieved using liquid nitrogen to cool the substrate to a nominal temperature of $77 \mathrm{~K}$. Correspondingly, the grain structures fall within the zone T and zone III regions[95]. The objective of the study is therefore to deposit and characterize sputtered indium on silicon at cryogenic temperatures to achieve a highly planar and closely packed indium layer thus drastically improving the quality of indium solder for electronic and optoelectronic devices.

At room-temperature, films were typically $8.6 \mu \mathrm{m}$ thick, while low temperature sputtering resulted in $4.8 \mu \mathrm{m}$ thick indium films. The main difference was due to indium packing density. The room temperature samples had a very rough morphology while the cryogenic samples were near specular. The reason for such an improvement in the quality of the samples can be seen in Figures 50(b) and 50(c) which show plan-view SEM images of the two samples [Figure 50(b) at $77 \mathrm{~K}$, Figure 50(c) at $300 \mathrm{~K}$ ]. For samples kept at $77 \mathrm{~K}$ during the sputtering process shows the presence of some large surface features, which are crystalline indium grains, with large areas of planar surfaces. The AFM image in Figure 50(a) shows a profile of the flatter surface and this measurement registers a root mean square roughness of $\sim 20 \mathrm{~nm}$. In contrast the silicon substrate that was kept at room temperature during the sputtering shows a much higher density of indium grains, with some features as long as $50 \mu \mathrm{m}$ and having facet formation. For room-temperature depositions, the film thickness is $\sim 7.3 \%$. Thus the growth of the sample at cryogenic temperatures has moved the growth mode into a much more planar regime compared to the room temperature sample. This effect is consistent with other metallic films structure zone model[95]. However the issue of the residual crystalline structures in the cryogenic sample needs to be understood. This can be explained through the more involved model by Thornton[96] where the observed surface is termed a "transitional surface" laying between a completely planar zone and a columnar growth mode zone. This issue may be resolved in the future by further reducing the homologous temperature by making use of a helium based cryogenic system or by modifying the argon plasma pressure both of which have been effective in other metal systems[97]. 


\subsection{Intracavity Down-conversion}

\subsubsection{Motivation}

The lack of high-power, tunable, efficient long-wave infrared (LWIR) sources is limiting active sensing technology and waveform agility, a critical need for LADAR type imaging. Most often, quantum cascade lasers are used for direct generation, which has only achieved about $100 \mathrm{~mW}$ with single-digit efficiencies and little tunability. Nonlinear processes most often uses rare-earth solid state lasers and PPLN, which have inefficient generation at longer wavelengths and have relatively narrow tuning bands. This bottleneck is removed by the open cavity design of the VECSELS providing access to high intracavity powers required for linewidth control and wavelength tunabilty through intracavity non-linear frequency mixing processes.

Based on this concept, a blue-green laser with high power and stability has been reported applying a tunable intracavity type II sum-frequency generation (SFG) in a folded T-cavity configuration.[98] However the critical defense need of accessing wavelengths in the MWIR and LWIR spectral region requires the application of intracavity difference frequency generation (DFG) processes. Herein we demonstrate the efficient generation of MWIR wavelengths applying intracavity DFG in a T-VECSEL cavity. We also provide a comparison of the experimentally obtained efficiencies with theoretically calculated values.

\subsubsection{Results and Discussion}

The T-VECSEL cavity consists of two laser cavities one of which is folded around a polarization beam splitter (PBS) generating orthogonal polarizations of the two lasers lasing at $980 \mathrm{~nm}$ and $1170 \mathrm{~nm}$ in the cavity as shown in figure $1 \mathrm{~b}$ and 1c. The regions between the PBS and "VECSEL chip 1" (s-polarization), and PBS and "VECSEL chip 2" (p-polarization) have orthogonal polarizations as depicted in the schematic in Figure 51. There is a collinear region from the PBS to a flat mirror via a high reflecting concave mirror where these two polarizations are multiplexed to satisfy a type II phase matching condition through a nonlinear crystal for DFG. Folding the collinear cavities at the common cout-coupler allows for the simultaneous focusing of the overlapped lasing modes into a nonlinear crystal thereby facilitating efficient difference frequency generation. The crystal is made of silver thiogallate $\left(\mathrm{AGS}\right.$ or $\mathrm{AgGaS}_{2}$ ) chosen due to its large effective optical nonlinearity, wide spectral and angular acceptance, broad transparency range and non-critical requirements for temperature stabilization and vibration control. The crystal offers a transparency range between 0.53-12 microns spanning over the frequency band of the DFG. The dimensions of the crystal are $5 \times 5 \times 5 \mathrm{~mm}^{3}$ cut at $\theta=42^{\circ}$ and $\phi=90^{\circ}$ in order to satisfy a type II phase matching condition.[99] Birefringent filters at Brewster's angles are used in front of each chip for independent tuning of wavelengths across its gain band. The experimentally generated DFG signal is depicted in Figure 52 showing a range of idler wavelengths between $5.3 \mu \mathrm{m}$ and $5.5 \mu \mathrm{m}$.

Based on the energy conservation law depicted in Figure 51a, a small tunability in nanometers in the pump wavelength (e.g.30 nm for the $980 \mathrm{~nm}$ chip), while keeping the signal constant at $1170 \mathrm{~nm}$ provides a tunability of several microns for the idler or the mid-IR wavelength as shown in Figure 51d. Similarly a small change in the phase matching angle in the crystal also gives rise to a broad tunability of the mid-IR wavelengths as depicted in Figure 51e. The phase matching condition for type II negative case where type II corresponds to the condition where pump and signal wavelengths have extraordinary (p) and ordinary (s) 

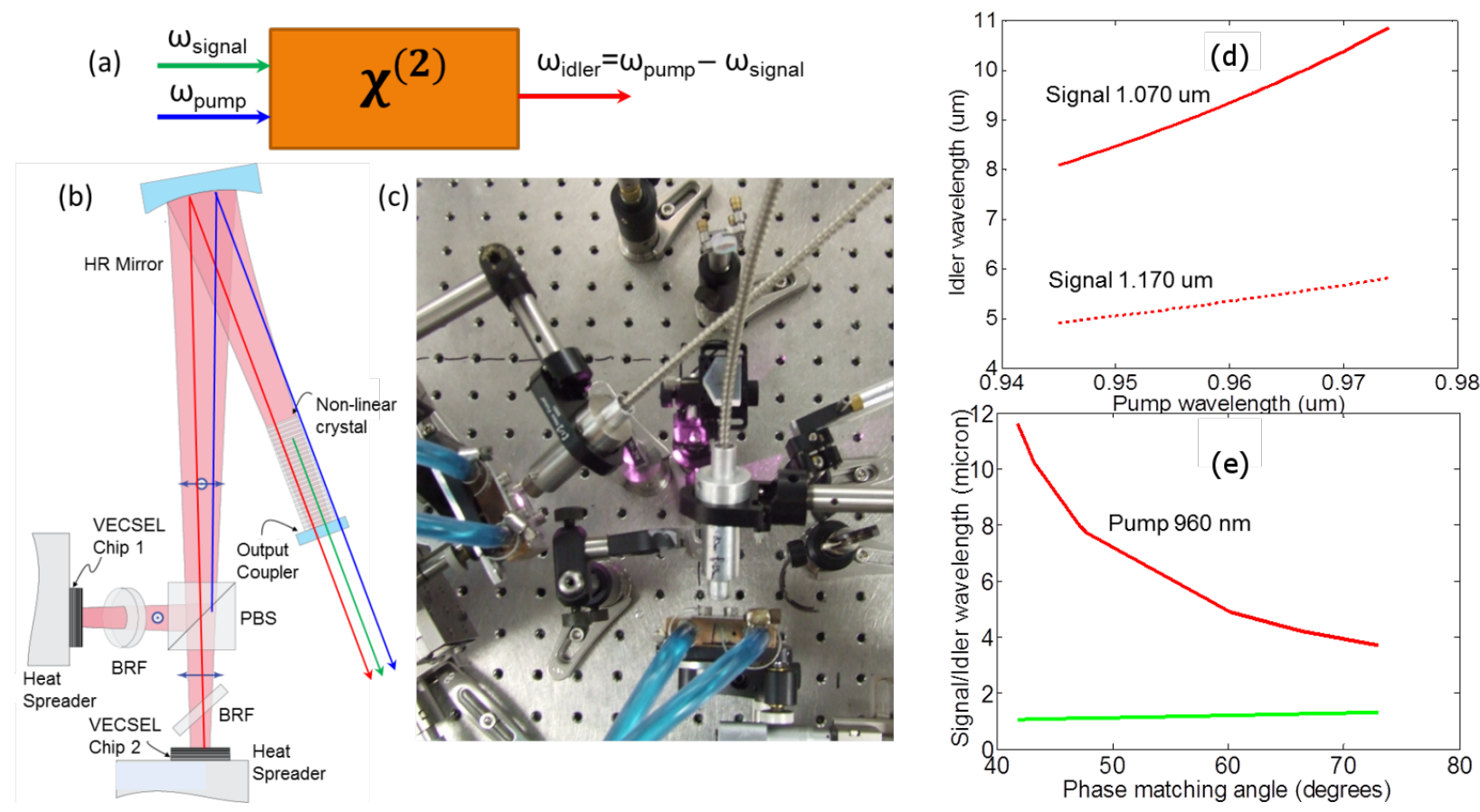

Figure 51. T-VECSEL schematic, experiment image, and theory

(a) Optical down-conversion process, (b) schematic of the T-VECSEL cavity depicting the various optical components. (c) Experimental set-up. (d) Plot showing the tunability of the idler wavelength with pump wave at constant signals $1.17 \mu \mathrm{m}$ (red dashed) and $1.07 \mu \mathrm{m}$ (red solid). (e) Phase matching curve depicting the tenability of the signal (green) and idler (red) wavelengths with phase matching angles in the crystal. 

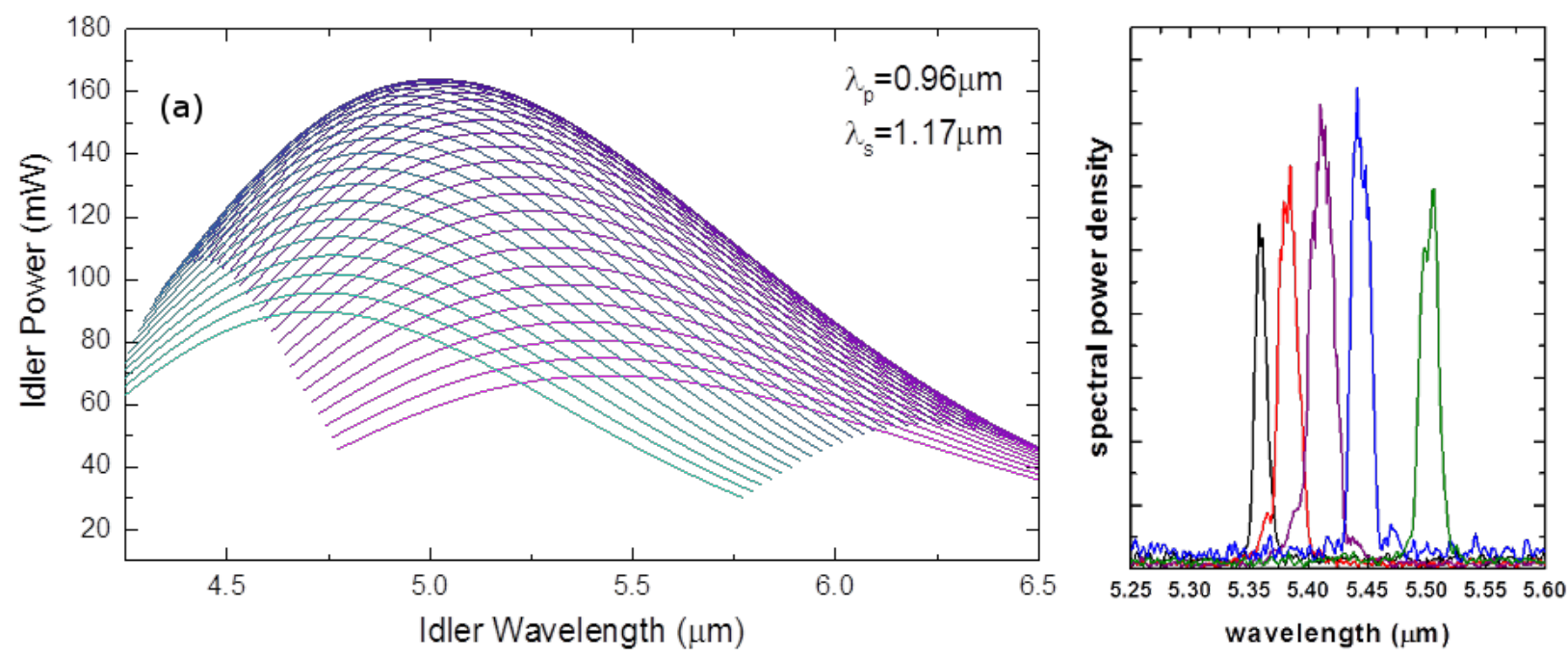

Figure 52. Theoretical/experimental DFG idler frequencies demonstrating tunability from 5.3 and $5.5 \mu \mathrm{m}$.

(a) Power tuning curve for pump and signal wavelengths centered at $960 \mathrm{~nm}$ and $1170 \mathrm{~nm}$ respectively. (b) experimental DFG.

polarizations respectively and negative refers to negative uniaxial crystal (AGS), is given by:[99]

$$
\frac{\cos ^{2} \theta_{p}}{\left(n_{p}^{o}\right)^{2}}+\frac{\sin ^{2} \theta_{p}}{\left(n_{p}^{e}\right)^{2}}=\left[\left(\frac{n_{s}^{o}}{\lambda_{s}}+\frac{n_{i}^{e}}{\lambda_{i}}\right)^{2} \times \lambda_{p}^{2}\right]^{-1}
$$

where $n_{p}^{o}, n_{p}^{e}, n_{s}^{o}$ and $n_{s}^{e}$ are the refractive indices for the ordinary (s) and extraordinary (p) polarizations for the pump and signal wavelengths respectively and $\theta_{p}$ is the angle that the incident pump wavevector makes with the optical axis of the crystal.

The corresponding power conversion efficiency is given by:[100]

$$
\eta_{i}=\frac{P_{i}}{P_{p}}=\frac{32 \pi^{2} d_{e f f}^{2} l P_{s} h}{\eta_{o} c \lambda_{s} \lambda_{i}^{2} n_{s} n_{p} n_{i}(1+\mu)}
$$

where $P_{i}, P_{p}$ and $P_{s}$ are the intensities of the idler, pump and signal wavelengths respectively, $l$ is the length of the crystal, $\mu$ is the ratio of the pump and signal wavelengths, $h$ is a parameter related to the beam waist and length of the crystal, $c$ is the speed of light in vacuum, et $a_{o}$ is the permittivity of free space, $n_{s}, n_{p}, n_{i}$ are the average refractive indices of the signal, pump and idler respectively. Finally, $d_{\text {eff }}$ is the effective non-linear coefficient of the crystal which is given as:

$$
d_{e f f} \equiv d_{36} \sin (2 \theta) \cos (2 \phi)
$$

A power tuning curve based on this formulation is depicted in Figure 52a showing maximum power conversion achieved at around $5 \mu \mathrm{m}$ which is in good agreement with the experiments (depicted in Figure 52b).

In summary, we have demonstrated the generation of mid-IR wavelengths using a unique method of non-linear DFG process applying semiconductor VECSELs in a T shaped optical cavity. A type II phase 
matching condition has been satisfied to generate these wavelengths. The peak power conversion efficiencies obtained experimentally around $5 \mu \mathrm{m}$ agree well with the theory. Furthermore there is a potential to obtain broad tunability, higher output powers, and extension to LWIR region with this method and is currently under investigation. Thus the semiconductor VECSEL technology in combination with a non-linear frequency mixing process has significant potential to obtain high power/brightness lasers with broad tunability providing access to wavelengths which are difficult to achieve with conventional sources thereby paving a promising technology route for future sensing and imaging applications. 


\subsection{CONCLUSIONS}

There are many summary comments that we can say about multiple efforts within this Work Unit. In the Type-I quantum-well efforts, we have shown that we can quantum design the Auger recombination through detailed band structure engineering. We experimentally realize three different laser structures with various barrier heights and analyzed the room temperature power performance and the and temperature-dependent threshold variation, for sources operating at 2 microns. We obtain an increase in the carrier lifetime of $>6 \times$. Our detailed theoretical analysis shows that most of this improvement is due to the improved Auger lifetime, with a lifetime as long as $\sim 5.7 \times$ that of the standard structure. Moreover, a similar concept has been utilized to theoretically show orders of magnitude improvements for lasers operating at wavelengths above $3 \mu \mathrm{m}$. Improvements to efficiency of laser devices can be very significant, although depends highly on operation regime.

We have established antimony nano-condensates as a mechanism for reduced reflectivity from MBE grown antimonide DBRs. We have analysed these DBRs using TEM and AFM and have concluded that these crystalline structures consist of antimony. A simulation of the reflectivity of the DBR with the serrated antimony features on the surface of the DBR agrees with the observed changes in the reflectivity spectrum. Finally an anneal process at $400{ }^{\circ} \mathrm{C}$ restores the reflectivity of the DBR to its intended design thus confirming the analysis.

Because in high power lasers, thermal management is of the utmost importance, we have spent some effort to create a new type of solder deposition, where we have deposited indium on silicon substrates using DC magnetron sputtering comparing samples at liquid nitrogen cryogenic temperature to those deposited at room temperature. The results of these experiments are consistent with structured zone models for metal sputtering with the lower homologous temperature which leads to a highly planar indium film with a near specular surface, while the higher homologous temperature results in very rough morphology dominated by micron size crystalline grains. The indium film on the cryogenic sample still has some remaining crystalline features which puts the deposition process in a transitional zone, between completely planar and completely rough morphologies, in Thornton et al.'s structure zone model. However the large planar regions have a very low RMS roughness of $\sim 20 \mathrm{~nm}$. By operating in a different zone region, it is worth noting that processing parameters such as pressure and plasma power may be revisited to achieve faster film depositions. Furthermore, we expect that this technique may be used to create smaller grain indium films with other deposition techniques such as evaporation. 


\subsection{REFERENCES}

[1] R. W. Russell, G. S. Rossano, M. A. Chatelain, D. K. Lynch, T. K. Tessensohn, E. Abendroth, D. Kim, and P. Jenniskens, "Mid-Infrared Spectroscopy of Persistent Leonid Trains," Earth Moon and Planets, vol. 82, pp. 439-456, 2000.

[2] F. E. Hanson, P. M. Poirier, E. J. Schimitschek, and M. A. Arbore, "Coherent mid-IR laser radar," in Proc. SPIE Vol. 4377, p. 155-162, Laser Radar Technology and Applications VI, 2001.

[3] M. Kuznetsov, F. Hakimi, R. Sprague, and A. Mooradian, "High-power ( $>0.5-\mathrm{w} \mathrm{cw}$ ) diode-pumped vertical-external-cavity surface-emiting semiconductor lasers with circular TEM $\mathrm{T}_{00}$ beams," IEEE Photon. Tech. Lett., vol. 9, no. 8, pp. 1063-1065, 1997.

[4] U. Keller and A. Tropper, "Passively modelocked surface-emitting semiconductor lasers," Physics Reports, vol. 429, pp. 67-120, 2006.

[5] L. R. Brovelli, U. Keller, and T. H. Chiu, "Design and operation of antiresonant fabry-perot saturable semiconductor absorbers for mode-locked solid-state lasers," J. Opt. Soc. Am. B, vol. 12, pp. 311-322, 1995.

[6] C. A. Zaugg, Z. Sun, V. J. Wittwer, D. Popa, S. Milana, T. S. Kulmala, R. S. Sundaram, M. Mangold, O. D. Sieber, M. Golling, Y. Lee, J. H. Ahn, A. C. Ferrari, and U. Keller, "Ultrafast and widely tuneable vertical-external-cavity-surface-emitting-laser, mode-locked by a graphene-integrated distributed bragg reflector," Opt. Express, vol. 21, no. 25, pp. 31548-31559, 2013.

[7] J. Faist, F. Capasso, D. L. Sivco, A. L. Hutchinson, S.-N. G. Chu, and A. Y. Cho, "Short wavelength $(\lambda \sim 3.4 \mu \mathrm{m})$ quantum cascade laser based on strained compensated ingaas/alinas," Applied Physics Letters, vol. 72, no. 6, pp. 680-682, 1998.

[8] M. Razeghi, A. Evans, S. Slivken, and J.-S.Yu, "High-power CW quantum cascade lasers: how short can we go?," vol. 5738, (USA), pp. 1 - 12, SPIE, 2005.

[9] D. G. Revin, J. W. Cockburn, M. J. Steer, R. J. Airey, M. Hopkinson, A. B. Krysa, L. R. Wilson, and S. Menzel, "InGaAs/AlAsSb/InP quantum cascade lasers operating at wavelengths close to $3 \mu \mathrm{m}$," Applied Physics Letters, vol. 90, no. 2, p. 021108, 2007.

[10] M. Nobile, H. Detz, A. M. Andrews, P. Klang, W. Schrenk, and G. Strasser, "An aluminum-free mid-infrared quantum cascade laser," vol. 7616, p. 76160M, SPIE, 2010.

[11] J. R. Lindle, C. S. Kim, M. Kim, W. W. Bewley, C. L. Canedy, I. Vurgaftman, and J. R. Meyer, "High-performance interband cascade lasers emitting in the 2.9-4.2 $\mu$ m wavelength range," vol. 7230, p. 72300R, SPIE, 2009.

[12] A. P. Ongstad, R. Kaspi, G. C. Dente, M. L. Tilton, R. Barresi, and J. R. Chavez, "Wavelength tuning limitations in optically pumped type-II antimonide lasers," Appl. Phys. Lett., vol. 92, no. 14, p. 141106, 2008. 
[13] A. Härkönen, M. Guina, O. Okhotnikov, K. Rößner, M. Hümmer, T. Lehnhardt, M. Müller, A. Forchel, and M. Fischer, "1-W antimonide-based vertical external cavity surface emitting laser operating at 2- $\mu$ m,” vol. 14, no. 14, pp. 6479-6484, 2006.

[14] L. Shterengas, G. Belenky, G. Kipshidze, and T. Hosoda, "Room temperature operated $3.1 \mu \mathrm{m}$ type-I GaSb-based diode lasers with $80 \mathrm{mw}$ continuous-wave output power," Applied Physics Letters, vol. 92 , no. 17, p. 171111, 2008.

[15] G. L. Belenky, J. G. Kim, L. Shterengas, A. Gourevitch, and R. U. Martinelli, "High-power $2.3 \mu \mathrm{m}$ laser arrays emitting $10 \mathrm{~W} \mathrm{CW}$ at room temperature," Electronics Letters, vol. 40, pp. 737 - 738, june 2004.

[16] G. Belenky, L. Shterengas, D. Wang, G. Kipshidze, and L. Vorobjev, "Continuous wave operated 3.2- $\mu \mathrm{m}$ type-I quantum-well diode lasers with the quinary waveguide layer," Semiconductor Science and Technology, vol. 24, no. 11, p. 115013, 2009.

[17] J. R. Meyer, C. L. Felix, W. W. Bewley, I. Vurgaftman, E. H. Aifer, L. J. Olafsen, J. R. Lindle, C. A. Hoffman, M.-J. Yang, B. R. Bennett, B. V. Shanabrook, H. Lee, C.-H. Lin, S. S. Pei, and R. H. Miles, "Auger coefficients in type-II InAs/Ga ${ }_{1-x} \mathrm{In}_{x}$ Sb quantum wells," Appl. Phys. Lett., vol. 73, no. 20, pp. 2857-2859, 1998.

[18] M. Lindberg and S. W. Koch, "Effective Bloch equations for semiconductors," Phys. Rev. B, vol. 38, pp. 3342-3350, Aug 1988.

[19] J. Hader, J. Moloney, and S. Koch, "Temperature dependence of radiative and Auger losses in quantum wells,” IEEE Journal of Quantum Electronics, vol. 44, pp. 185 -191, feb. 2008.

[20] S. Adachi, "Band gaps and refractive indices of AlGaAsSb, GaInAsSb, and InPAsSb: Key properties for a variety of the 2-4 $\mu$ m optoelectronic device applications," Journal of Applied Physics, vol. 61, no. 10, pp. 4869-4876, 1987.

[21] G. P. Agrawal and N. K. Dutta, Semiconductor Lasers. Norwell, MA: Kluwer Academic Publishers, second ed., 1993.

[22] A. S. Polkovnikov and G. G. Zegrya, "Auger recombination in semiconductor quantum wells," Phys. Rev. B, vol. 58, pp. 4039-4056, Aug 1998.

[23] J. E. Ayers, Heteroepitaxy of Semiconductors: Theory, Growth, and Characterization, ch. 2: Properties of semiconductors, pp. 7-74. CRC Press, 2007.

[24] T. W. Butler, "On the determination of dislocation densities," Tech. Rep. Rep.E-69-1, USNA Eng. Dept., May 1969.

[25] R. Bedford, “Tunable high-brightness semiconductor sources (February-May 2011).”.

[26] R. G. Bedford, G. Triplett, D. H. Tomich, S. W. Koch, J. Moloney, and J. Hader, "Reduced auger recombination in mid-infrared semiconductor lasers," Journal of Applied Physics, vol. 110, p. 073108, 2011. 
[27] E. A. Pease, L. R. Dawson, L. G. Vaughn, P. Rotella, and L. F. Lester, "2.5-3.5 $\mu$ m optically pumped GaInSb/AlGaInSb multiple quantum well lasers grown on AlInSb metamorphic buffer layers," Journal of Applied Physics, vol. 93, no. 6, pp. 3177-3181, 2003.

[28] M. Rattunde, M. Kelemen, N. Schulz, C. Pfahler, C. Manz, J. Schmitz, G. Kaufel, and K. Wagner, "High-brightness 2.x $\mu \mathrm{m}$ semiconductor lasers," in Mid-Infrared Coherent Sources and Applications (M. Ebrahim-Zadeh and I. Sorokina, eds.), pp. 193-224, 2008.

[29] W. W. Bewley, C. L. Felix, I. Vurgaftman, D. W. Stokes, L. J. Olafsen, E. H. Aifer, J. R. Meyer, M. J. Yang, B. V. Shanabrook, H. Lee, R. U. Martinelli, J. C. Connolly, and A. R. Sugg, "High-temperature continuous-wave operation of optically-pumped type-II W lasers from 3-6.3 $\mu \mathrm{m}$,” p. 366, may 1999.

[30] G. Rex, O. Ongstad, M. Tilton, G. Dente, D. Gianardi, R. Barresi, J. Chavez, and R. Kaspi, "Mid-IR semiconductor lasers," jan 2010. unclassified // for official use only.

[31] J. R. Lindle, C. S. Kim, M. Kim, W. W. Bewley, C. L. Canedy, I. Vurgaftman, and J. R. Meyer, "High-performance interband cascade lasers emitting in the 2.9-4.2 $\mu$ m wavelength range," vol. 7230, p. 72300R, SPIE, 2009. RGB note: approximate maximum powers based on threshold and slope and "typical" roll-over due to thermal effects.

[32] G. C. Dente, M. L. Tilton, A. P. Ongstad, and R. Kaspi, "Wavelength tuning predictions and experiments for type II antimonide lasers," J. of Appl. Phys., vol. 103, no. 2, pp. 023106 - 1, 2008.

[33] R. Noll and S. Macomber, “Analysis of grating surface emitting lasers,” IEEE Journal of Quantum Electronics, vol. 26, pp. 456 -466, mar 1990.

[34] N. Finger, W. Schrenk, and E. Gornik, "Analysis of TM-polarized DFB laser structures with metal surface gratings," IEEE Journal of Quantum Electronics, vol. 36, pp. 780 -786, jul 2000.

[35] R. Kaspi, A. Ongstad, G. C. Dente, J. Chavez, M. L. Tilton, and G. Gianardi, "High power and high brightness from an optically pumped InAs/InGaSb type-II mid-infrared laser with low confinement," Appl. Phys. Lett., 2002.

[36] R. Bedford, “Tunable high-brightness semiconductor sources (October/November 2011).”.

[37] R. Bedford, “Tunable high-brightness semiconductor sources (December/January 2011).”.

[38] A. S. Marathay and J. F. McCalmont, "On the usual approximation used in the Rayleigh-Sommerfeld diffraction theory,” J. Opt. Soc. Am. A, vol. 21, pp. 510-516, Apr 2004.

[39] Z. Sun, T. Hasan, F. Torrisi, D. Popa, G. PRivitera, F. Wang, F. Bonaccorso, D. M. Basko, and A. C. Ferrari, "Graphene mode-locked ultrafast laser," ACS Nano, vol. 4, p. 803, 2010.

[40] Q. Bao, H. Zhang, Y. Wang, Z. Ni, Y. Yan, Z. Shen, K. P. Loh, and D. Y. Tang, "Atomic-layer graphene as a saturable absorber for ultrafast pulsed lasers," Adv. Funct. Mater., vol. 19, pp. 3077-3083, 2009.

[41] X. LI, J. Xu, Y. Wu, J. He, and X. Hao, "Large energy laser pulses with high repetition rate by graphene q-switched solid-state laser,” Opt. Express, vol. 19, pp. 9950-9955, 2011. 
[42] I. H. Baek and et. al, "Efficient mode-locking of sub-70-fs ti:sapphire laser by graphene saturable absorber,” Appl. Phys. Express, vol. 5, pp. 032701-3, 2012.

[43] D. P. et al, "Graphene q-switched tunable fiber laser," Appl. Phys. Lett., vol. 98, p. 073106, 2011.

[44] Z. Sun, T. Hasan, and A. C. Ferrari, "Ultrafast lasers mode-locked by nanotubes and graphene," Physica E., vol. 44, pp. 1082-1091, 2012.

[45] P. Dutta, H. Bhat, and V. Kumar, "The physics and technology of gallium antimonide: an emerging optoelectronic material,” J. of Appl. Phys., vol. 81, no. 9, pp. 5821-5870, 1997.

[46] F. Bonaccorso, Z. Sun, T. Hasan, and A. C. Ferrari, "Graphene photonics and optoelectronics," Nat. Phot., vol. 4, 2010.

[47] L. W. Tutt and T. F. Boggess, "A review of optical limiting mechanisms and devices using organics, fullerenes, semiconductors and other materials," Prog. Quant. Electr, vol. 17, p. 299, 1993.

[48] Z. Liu, Y. Wang, X. Zhang, Y. Xu, Y. Chen, and J. Tian, "Nonlinear optical properties of graphene oxide in nanosecond and picosecond regimes," Appl. Phys. Lett., vol. 94, pp. 021902(1-3), 2009.

[49] M. Feng, H. Zhan, and Y. Chen, "Nonlinear optical and optical properties of graphene families," Appl. Phys. Lett., vol. 96, pp. 033107(1-3), 2010.

[50] K. Mansour, M. J. Soileau, and E. W. V. Stryland, "Nonlinear optical properties of carbon-black suspensions(ink)," J. Opt. Soc. Am. B., vol. 9, pp. 1100-9, 1992.

[51] G. K. Lim, Z. L. Chen, J. Clark, R. G. S. Goh, W. H. Ng, H. W. Tan, R. H. Friend, P. K. H. Ho, and L. Chua, "Giant broadband nonlinear optical absorption response in dispersed graphene single sheets," Nat. Photon., vol. 5, pp. 554-60, 2011.

[52] X. Zheng, M. Feng, Z. Li, Y. Song, and H. Zhan, "Enhanced nonlinear optical properties of nonzero-bandgap graphene materials in glass matrices," J. Mater. Chem. C, 2014.

[53] C. Liu, Y. Chang, T. B. Norris, and Z. Zhong, "Graphene photodetectors with ultra-broadband and high responsivity at room temperature,” Nat. Nanotech, vol. 9, pp. 273-278, 2014.

[54] T. Mueller, F. Xia, and P. Avouris, "Graphene photodetectors for high speed optical communications," Nat. Photon, vol. 3, pp. 297-301, 2010.

[55] W. Li, B. Chen, W. F. C. Meng, Y. Xiao, X. Li, Z. Hu, Y. Xu, L. Tong, H. Wang, W. Liu, J. Bao, and Y. R. Shen, “Ultrafast all-optical graphene modulator,” Nano. Lett., vol. 14, no. 2, pp. 955-959, 2014.

[56] X. Zhao, Z. B. Li, W. B. Yan, Y. Wu, X. L. Xhang, Y. Chen, and J. G. Tian, "Ultrafast carrier dynamics and saturable absorption of solution-processible few-layered graphene oxide," Appl. Phys. Lett., vol. 98, pp. 121905(1-3), 2011.

[57] Y. Zhu, S. Murali, W. Cai, X. Li, J. W. Suk, J. R. Potts, and R. S. Ruoff, "Graphene and graphene oxide: Synthesis, properties and applications,” Adv. Mater., vol. 22, pp. 3906-3924, 2010. 
[58] S. Saxena, T. A. Tyson, S. Shukla, E. Negusse, H. Chen, and J. Bai, "Investigation of structural and electronic properties of graphene oxide,” Appl. Phys. Lett., vol. 99, pp. 013104 (1-3), 2011.

[59] A. C. Ferrari and J. Robertson, "Raman spectroscopy of amorphous, nanostructured, diamond- like carbon, and nanodiamond,” Phil. Trans. R. Soc. Lond. A., vol. 362, pp. 2477-2512, 2004.

[60] L. M. Malard, J. Nilsson, D. C. Elias, J. C. Brant, F. Plentz, E. S. Alves, A. H. C. Neto, and M. A. Pimenta, "Probing the electronic structure of bilayer graphene by raman scattering," Phys. Rev. B., vol. 76, pp. 201401(1-4), 2007.

[61] D. S. Lee, C. Riedl, B. Krauss, K. von Klitzing, U. Starke, and J. H. Smet, "Raman spectra of epitaxial graphene on sic and of epitaxial graphene transferred to sio2," Nano. Lett., vol. 8, pp. 4320-25, 2008.

[62] L. Vivien, D. Riehl, F. Hache, and E. Anglaret, "Optical limiting properties of carbon nanotubes," Physica B, vol. 323, pp. 233-34, 2002.

[63] P. Chen, X. Wu, X. Sun, J. Lin, W. Ji, and K. L. Tan Phys. Rev. Lett., vol. 82, pp. 2548-51, 1999.

[64] S. R. Mishra, H. S. Rawat, S. C. Mehendale, K. C. Rustagi, A. K. Sood, R. Bandyopadhyay, A. Govindaraj, and C. Rao, "Optical limiting in single walled carbon nanotube suspensions," Chem. Phys. Lett., vol. 317, pp. 510-14, 2000.

[65] F. E. Hernandez, W. S. III, I. Cohanoschi, D. J. Hagan, and E. W. V. Stryland, "Viscosity dependence of optical limiting in carbon-black suspensions," Appl. Opt., vol. 41, pp. 1103-7, 2002.

[66] Z.Liu, X. Zhang, X. Yan, Y. Chen, and J. Tian, "Nonlinear optical properties of graphene-based materials," Chin. Sci. Bull., vol. 57, pp. 2971-2982, 2012.

[67] X. Zhang, Z. Liu, X. Li, Q. Ma, X. Chen, J. Tian, Y. Xu, and Y. Chen, "Transient thermal effect, nonlinear refraction and nonlinear absorption properties of graphene oxide sheets in dispersion," Opt. Express, vol. 21, no. 6, p. 7511, 2013.

[68] M. B. M. Krishna, N. Venkatramaiah, and D. N. Rao, "Optical transmission control in graphene oxide and its organic composites with ultrashort laser pulses," J. Opt, vol. 16, p. 015205, 2014.

[69] L. Wang, J. Yoshida, N. Ogata, S. Sasaki, and T. Kajiyama, "Self-assembled supramolecular films derived from marine deoxyribonucleid acid (DNA) - cationic surfactant complexes: Large-scale preparation and optical and thermal properties," Chem. Mater., vol. 13, no. 4, p. 1273, 2001.

[70] J. A. Hagen, W. Li, A. J. Steckl, and J. G. Grote, "Enhanced emission efficiency in organic light emitting diodes using deoxyribonucleic acid complex as an electronic blocking layer," Appl. Phys. Lett, vol. 88, p. 171109, 2006.

[71] E. M. Heckman, J. G. Grote, F. K. Hopkins, and P. P. Yaney, "Performance of an electo-optic waveguide modulator fabricated using a deoxyribonucleic acid-based biopolymer," Appl. Phys. Lett, vol. 89, p. 181116, 2006.

[72] P. P. Yaney, E. M. Heckman, D. E. Diggs, F. K. Hopkins, and J. G. Grote, "Development of chemical sensors using polymer optical waveguides fabricated with DNA,” Proc. SPIE, vol. 5724, p. 224, 2005. 
[73] F. Ouchen, G. A. Sotzing, T. L. Miller, K. M. Singh, B. A. Telek, A. C. Lesko, R. Aga, E. M. Fehrman-Cory, P. P. Yaney, J. G. Grote, C. M. Bartsch, and E. M. Heckman, "Modifield processing techniques of a DNA biopolymer for enhanced performance in photonics applications," Appl. Phys. Lett, vol. 101, p. 153702, 2012.

[74] F. Ouchen, P. P. Yaney, and J. G. Grote, "DNA biocompatible materials," Proc. SPIE, vol. 7403, p. 74030F, 2009.

[75] L. David, A. Feldman, E. Mansfield, J. Lehman, and G. Singh, "Evaluating the thermal damage resistance of graphene/carbon nanotube hybrid composite coatings," Sci. Reports, vol. 4, p. 4311, 2014.

[76] M. Currie, J. D. Caldwell, F. J. Bezares, J. Robinson, T. Anderson, H. Chun, and M. Tadjer, “Quantifying pulsed laser induced damage to graphene,” Appl. Phys. Lett., vol. 99, p. 211909, 2011.

[77] B. C. A. Das and A. K. Sood, "Raman spectroscopy of graphene on different substrates and influence of defects," Bull. Mater. Sci, vol. 31, p. 579, 2008.

[78] A. C. Ferrari, J. C. Meyer, V. Scardaci, C. Casiraghi, M. Lazzeri, F. Mauri, S. Piscanec, D. Jiang, K. S. Novoselov, S. Roth, and A. K. Geim, "Raman spectrum of graphene and graphene layers," Phys. Rev. Lett., vol. 97, p. 187401, 2006.

[79] L. M. Malard, M. A. Pimenta, G. Dresselhaus, and M. Dresselhaus, "Raman spectroscopy in graphene," Phys. Reports, vol. 473, pp. 51-87, 2009.

[80] M. S. Sheikh-Bahae, A. A. Said, T. Wei, D. J. Hagan, and E. W. van Stryland, "Sensitive measurement of optical nonlinearities using a single beam,” IEEE. J. of Quantum. Electron., vol. 26, pp. 760-769, 1990.

[81] Y. Gao, X. Zhang, Y. Li, H. Liu, Y. Wang, Q. Ching, W. Jiao, and Y. Song, "Saturable absorption and reverse saturable absorption in platinum nanoparticles," Optics Comm., vol. 251, p. 429, 2005.

[82] B. S. Kalanoor, P. B. Bisht, S. A. Ali, T. T. Baby, and S. Ramaprabhu, "Optical nonlinearity of silver-decorated graphene,” J. Opt. Soc. Am. B, vol. 29, pp. 669-675, 2012.

[83] G. Xing, H. Guo, X. Zhang, T. C. Sum, and C. H. A. Huan, "The physics of ultrafast saturable absorption in graphene,” Opt. Express, vol. 18, pp. 4564-4573, 2010.

[84] B. Heinen, T. Wang, M. Sparenberg, A. Weber, B. Kunert, J. Hader, S. Koch, J. Moloney, M. Koch, and W. Stolz, "106 w continuous-wave output power from vertical-external-cavity surface-emitting laser,” Electron. Lett., vol. 48, p. 516517, 2012.

[85] A. R. et al, "Transferring and identification of single-and few-layered graphene on arbitrary substrates," J. Phys. Chem. C, vol. 112, pp. 17741-17744, 2008.

[86] X. L. et al, "Large-area synthesis of high-quality and uniform graphene films on copper foils," Science, vol. 324, pp. 1312-1314, 2009.

[87] R. P. Howson, T. J. Fox, and D. C. Emmony, "Optical properties of thin films of antimony," J. Phys. D. Appl. Phys., vol. 7, no. 13, pp. 1864-1872, 1974. 
[88] K. Mizuishi, "Some aspects of bonding solder deterioration observed in long-lived semiconductor lasers: Solder migration and whisker growth,” J. of Appl. Phys., vol. 55, pp. 289 -295, Jan 1984.

[89] J. Jiang, S. Tsao, T. Sullivan, M. Razeghi, and G. J. Brown, "Fabrication of indium bumps for hybrid infrared focal plane array applications," Infrared Phys. Techn., vol. 45, no. 2, pp. 143 - 151, 2004.

[90] L. Fan, C. Hessenius, M. Fallahi, J. Hader, H. Li, J. V. Moloney, W. Stolz, S. Koch, J. T. Murray, and R. Bedford, "Highly strained InGaAs/GaAs multiwatt vertical-external-cavity surface-emitting laser emitting around 1170 nm,” Applied Physics Letters, vol. 91, p. 131114, 2007.

[91] F. Walsh and D. Gabe, “The electrodeposition of indium," Surf. Technol., vol. 8, no. 2, pp. 87 - 99, 1979.

[92] B. Natarajan, A. Eltoukhy, J. Greene, and T. Barr, "Mechanisms of reactive sputtering of indium i: Growth of inn in mixed ar-n2 discharges," Thin Solid Films, vol. 69, no. 2, pp. $201-216,1980$.

[93] M. Yudasaka, T. Matsuoka, and K. Nakanishi, "Indium selenide film formation by the double-source evaporation of indium and selenium," Thin Solid Films, vol. 146, no. 1, pp. 65 - 73, 1987.

[94] N. Zhu, "Thermal impact of solder voids in the electronic packaging of power devices," 15th Ann. IEEE SEMI-THERM Symp., pp. 22-29, March 1999.

[95] C. Grovenor, H. Hentzell, and D. Smith, "The development of grain structure during growth of metallic films," Acta Metall. Mater., vol. 32, no. 5, pp. 773 - 781, 1984.

[96] J. A. Thornton, "High rate thick film growth," Annu. Rev. Mater. Sci., vol. 7, no. 1, pp. 239-260, 1977.

[97] J. H. Park, "Microstructure development and evolution of sputter deposited indium thin films in cryogenics," Master's thesis, Auburn University, 2007.

[98] C. Hessenius, M. Lukowski, and M. Fallahi, "Tunable type-ii intracavity sum frequency generation in a two chip colinear vertical external cavity surface emitting laser," Opt. Lett., vol. 38, no. 5, pp. 640-642, 2013.

[99] R. Boyde, Nonlinear Optics. Academic Press, 2003.

[100] W. Shi, Y. Ding, N. Fernelius, and K. Vodopyanov, "Efficient, tunable, and coherent 0.18-5.27-Thz source based on GaSe crystal,” Opt. Lett., vol. 27, no. 16, pp. 1454-1456, 2002. 


\title{
List of Symbols, Abbreviations, and Acronyms
}

\author{
Acronym Description \\ AFM atomic force microscopy. 11, 12, 21, 44, 57 \\ AFRL/RY Sensors Directorate. 2, 3, 25 \\ AlAs aluminum arsenide. 26 \\ $\mathrm{AlSb} \quad$ aluminum antimonide. 10, 12 \\ CVD chemical vapor deposition. 63 \\ DBR distributed Bragg reflector. 2, 15, 18, 59, 63 \\ DFB distributed feedback. 29 \\ DFG difference-frequency generation. 1, 71, 73 \\ DoD Department of Defense. 2 \\ DQE differential quantum efficiency. 33, 37 \\ FET field effect transistor. 57 \\ FWHM full width half maximum. 37, 48, 64, 65 \\ G-DNA graphene - DNA. iv, 56, 57 \\ G-PVA graphene - poly vinyl alcohol compopsite. 55 \\ G-SAM graphene saturable absorber mirror. 58, 59, 61-64, 66 \\ GaAs gallium arsenide. 15, 18, 20, 21, 35, 37, 47 \\ GaSb gallium antimonide. 4, 10-12, 15, 18, 20-22, 37, 40, 43, 44 \\ HR-XRD high-resolution X-ray diffraction. iii, 11, 12 \\ ICL interband cascade laser. 25, 26 \\ ICP inductively-coupled plasma reactive ion etch. 43 \\ IMF interfacial misfit. 15, 16, 18, 21 \\ InAs indium arsenide. 10 \\ InP indium phosphide. 20 \\ IRCM infrared countermeasures. 2, 3 \\ LADAR laser radar. 2, 3 \\ LED light-emitting diode. 57 \\ LWIR long-wave infrared. 71, 74 \\ MBE molecular beam epitaxy. 9-11,37 \\ MOVPE metal-organic vapor phase epitaxy. 62 \\ MWIR mid-wave infrared. 1-3, 10, 15, 71 \\ NLA nonlinear absorption. 48, 49, 51, 52, 57 \\ NLCSTR Nonlinear Control Strategies, Inc.. 5, 20, 25 \\ NLR nonlinear refraction. 48, 49, 51, 52 \\ NLS nonlinear scattering. 45, 54
}




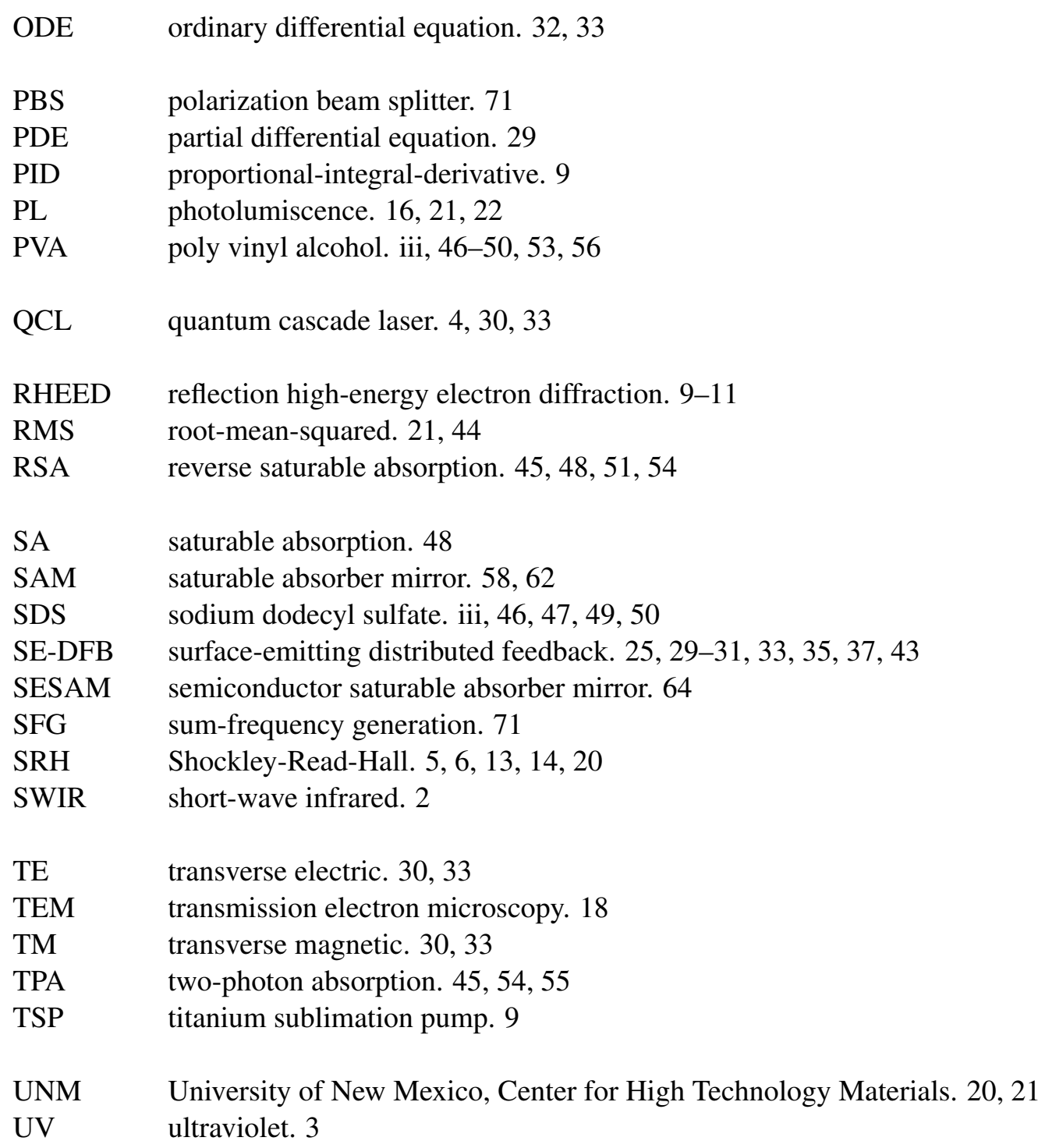

VCSEL vertical cavity surface emitting laser. 2

VECSEL vertical external cavity surface emitting laser. 2, 3, 15, 18, 40, 45, 61-65, 71, 73, 74 